\title{
Continuous Breathing Rare-Earth MOFs based on Hexanuclear Clusters with
}

Gas Trapping Properties

Giasemi K. Angeli, Edward Loukopoulos, Konstantinos Kouvidis, Artemis Bosveli, Constantinos Tsangarakis, Emmanuel Tylianakis, George Froudakis and Pantelis N. Trikalitis

Department of Chemistry, University of Crete, Voutes 71003 Heraklion, Greece

\section{SUPPORTING INFORMATION}

\section{Table of Contents}

Methods and instrumentation

Organic synthesis and ${ }^{1} \mathrm{H}$ NMR spectra

MOF synthesis

Scanning electron microscopy images (SEM)

Single crystal X-ray crystallography ...... S10

Additional structural figures

Topological analysis

Powder X-ray diffraction measurements

Gas and vapor sorption measurements

${ }^{1} \mathrm{H}$ NMR measurements of acid digested samples

Thermal gravimetric analyses (TGA) 


\section{Methods and Instrumentation}

Starting Materials All chemicals were purchased commercially and were used without further purification. Hydrochloric acid 37\%, $\mathrm{Pd}\left(\mathrm{PPh}_{3}\right)_{4}, \mathrm{H}_{2} \mathrm{SO}_{4}$, acetone $99.5 \%$, dichloromethane $\mathrm{CH}_{2} \mathrm{Cl}_{2}$, > $99.9 \%$, tetrahydrofuran (THF) $>99.9 \%$, methanol $(\mathrm{MeOH})>99.8 \%$, Ethanol absolute (EtOH), $\mathrm{NaOH}$ $98 \%$ pellets, $\mathrm{N}, \mathrm{N}$-dimethylformamide $99.8 \%$, and potassium disulfate were purchased from Aldrich. 1,3,5-tribromobenzene, $\mathrm{Y}\left(\mathrm{NO}_{3}\right)_{3} .6 \mathrm{H}_{2} \mathrm{O} 99.9 \%, \mathrm{Er}\left(\mathrm{NO}_{3}\right)_{3} .5 \mathrm{H}_{2} \mathrm{O} 99.9 \%, \mathrm{Ho}\left(\mathrm{NO}_{3}\right)_{3} .5 \mathrm{H}_{2} \mathrm{O} 99.9 \%$, $\mathrm{Eu}\left(\mathrm{NO}_{3}\right)_{3} \cdot 6 \mathrm{H}_{2} \mathrm{O} 99.9 \%, \mathrm{Sm}\left(\mathrm{NO}_{3}\right)_{3} \cdot 6 \mathrm{H}_{2} \mathrm{O} 99.9 \%, \operatorname{Dy}\left(\mathrm{NO}_{3}\right)_{3} \cdot 5 \mathrm{H}_{2} \mathrm{O} 99.9 \%, \mathrm{~Tb}\left(\mathrm{NO}_{3}\right)_{3} \cdot 6 \mathrm{H}_{2} \mathrm{O} 99.9 \%$ and boronic acid were purchased from Alfa Aesar. 2-fluorobenzoic acid was purchased from Fluorochem.

Powder X-Ray Diffraction Patterns were collected using a Panalytical X'pert Pro MPD System Cu Ka $(\lambda=1.5418 \mathrm{~A})$ radiation operated at $45 \mathrm{kV}$ and $40 \mathrm{~mA}$. A typical scan rate was $1.2 \mathrm{sec} / \mathrm{step}$ with a step size of 0.02 deg.

Scanning electron microscopy (SEM) images were collected on a JEOL JSM-6390LV microscope.

\section{Single Crystal X-ray Diffraction}

Single crystal X-ray diffraction data were collected on a Bruker D8 Venture diffractometer equipped with a $\mathrm{Cu}$ Incoatec microfocus I $\mu \mathrm{S} 3.0$ source, a Photon II detector operating in shutterless mode and a cryostrem 800 system (Oxford Cryosystems) for temperature regulation.

Thermogravimetric analyses (TGA) were performed using a Shimadzu TA-50 instrument. An amount of $11.8 \mathrm{mg}$ of sample was placed inside a quartz cap and heated up to $750^{\circ} \mathrm{C}$ under $\mathrm{N}_{2}$ flow with a heating rate of $5{ }^{\circ} \mathrm{C} / \mathrm{min}$.

${ }^{1} \mathbf{H}$ NMR spectra were recorded on a $500 \mathrm{MHz}$ Bruker spectrometer. MOF samples were prepared by digesting a small portion ( $2 \mathrm{mg})$ of the activated solids with a drop of concentrated $\mathrm{HCl}$ acid $(37 \%)$ in a DMSO- $d_{6}$ solution.

Gas sorption measurements. Low Pressure nitrogen, argon, carbon dioxide, methane, xenon and krypton gas sorption measurements were carried at different temperatures up to 1 bar using a stateof-the-art Autosorb-iQ2 instrument from Quantachrome equipped with a cryocooler system capable of temperature control from 20 to $320 \mathrm{~K}$. Prior to analysis the as made sample was washed with $\mathrm{N}, \mathrm{N}-$ dimethylformamide four times per day for 2 days to remove any unreacted starting materials from the pores. Then the sample was soaked in warm ethanol $\left(40^{\circ} \mathrm{C}\right)$ over a period of 4 days, replenishing the ethanol 4 times per day. Finally, the wet sample was transferred to a $6 \mathrm{~mm}$ sample cell using a pipette and activated under dynamic vacuum at different temperatures for 12 hours until the outgas rate was less than $2 \mathrm{mTorr} / \mathrm{min}$. After evacuation, the sample was re-weighed to obtain its precise mass and the cell was transferred to the analysis port of the gas adsorption instrument. Regarding the Ar isotherms recorded at temperatures above its boiling point $(87.3 \mathrm{~K})$, the saturation pressure $\left(p_{o}\right)$ at each measurement temperature was used to calculate the $p / p_{o}$ values shown in the $x$-axis. This allows for a direct comparison between isotherms recorded at different temperatures.

Vapor sorption measurements. Vapor sorption isotherms for $\mathrm{EtOH}, \mathrm{CH}_{3} \mathrm{CN}, \mathrm{C}_{6} \mathrm{H}_{6}$ and $\mathrm{n}-\mathrm{C}_{6} \mathrm{H}_{14}$ were recorded at different temperatures up to 1 bar, using a state-of-the-art, high precision instrument, BELSORP-maxll from Microtrac MRB, equipped with four (4) analysis stations and a specially designed detachable thermostatic bath for accurate measurements. Samples were activated in situ using a 4-position heater, at different temperatures for 12 hours under ultrahigh vacuum $\left(<1 \times 10^{-5}\right.$ $\mathrm{Pa}$ ). Prior to measurements, each vapor was degassed to remove any dissolved gases following a standard protocol. For comparison purposes, all isotherms are presented as the amount adsorbed as a function of the relative pressure, $p / p_{0}$, where $p_{o}$ is the saturation pressure of the vapor at the measurement temperature. 


\section{Theoretical calculations}

Grand Canonical Monte Carlo, GCMC, simulations were conducted in order to explore the capacity of both open and close structures to adsorb nitrogen. RASPA ${ }^{1}$ code was used for the simulations. Isotherms were calculated at $77 \mathrm{~K}$ and for a wide pressure range up to 1 bar. 100000 equilibration cycles were used for GCMC simulations followed by 100000 cycles for calculating the average properties. Each cycle was composed of a number of steps equal to the number of guest molecules present in the simulation cell at that time and this number of steps per cycle was at least 20. The periodic simulation cell was used large enough to ensure that its dimensions were at least two times the potential cut off distance i.e. $12.8 \AA$. Ewald summation was used to treat the electrostatic interactions.

The simulation cell included not only the MOF framework but dimethyl ammonium, DMA, cations. These cations served as counterions to the negatively charged metal node and the same time to represent accurately the material. Both Nitrogen molecules and DMA cations were allowed to translate and rotate whereas framework atoms were kept frozen at their crystallographic positions. Van der Waals and electrostatic interactions between host and guest molecule atoms were taken into account using the 6-12 Lennard Jones and Coulomb potential, respectively. Nitrogen molecule was described using a three center model, one for each atom and an extra one on the center of mass of the molecule. The two atoms were separated with a distance of $1.1 \AA$. To the central atoms were assigned parameters $\varepsilon=36 \mathrm{~K}$ and $\sigma=3.31 \AA$ whereas point charges of $2 q$ and $-q$ were placed on atom positions and center of mass respectively with $q=0.4048$ in an effort to describe the quadrupole moment of the molecule. Parameters $\varepsilon$ and $\sigma$ for framework atoms were taken from UFF forcefield ${ }^{2}$ and their point charges were calculated using CHELPEG $^{3}$ method which was applied on separate clusters for metal node and the various linkers. DMA cations were descried in atomistic detail with the geometry taken from optimization applying QM calculations in the DFT level of theory using RB3LYP functional and Def2TZVP basis set. Partial charges were then calculated using CHELPG method on the optimized geometry. ORCA ${ }^{4}$ software was used for the QM calculations. For cross parameters between different atom types Lorenz-Berthelot mixing rules were applied. 


\section{Organic synthesis and ${ }^{1} \mathrm{H}$ NMR spectra}<smiles>COC(=O)c1ccc(B(O)O)cc1</smiles>

eq

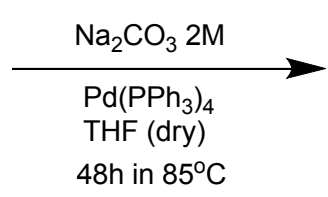<smiles>COC(=O)c1ccc(-c2cc(Br)cc(-c3ccc(C(=O)OC)cc3)c2)cc1</smiles>

1<smiles>O=C(O)c1cc(B(O)O)cc(C(=O)O)c1</smiles>

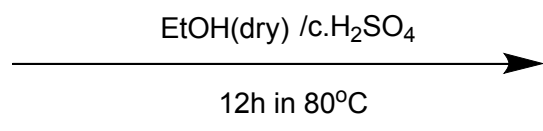

HOOC<smiles>O=C(O)c1ccc(-c2cc(-c3ccc(C(=O)O)cc3)cc(-c3cc(C(=O)O)cc(C(=O)O)c3)c2)cc1</smiles>

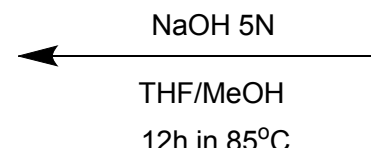

4

Chemical Formula: $\mathrm{C}_{28} \mathrm{H}_{18} \mathrm{O}_{8}$ Molecular Weight: 482.44

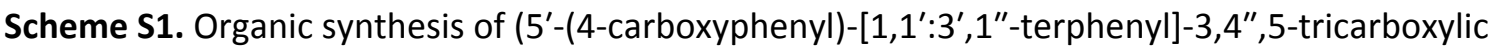
acid), $\mathrm{H}_{4}$ CPTTA $\left(\mathrm{H}_{4} \mathrm{~L}\right)$.

Compound 1: In a dried under Ar double neck round bottom flask, $0.5 \mathrm{~g}$ of 1,3,5-tribromobenzene (1.59 mmol) were added along with $40 \mathrm{ml}$ dry THF, $0.572 \mathrm{~g}(3.18 \mathrm{mmol})$ boronic acid, $15 \mathrm{ml} \mathrm{Na} \mathrm{CO}_{3}$ $2 \mathrm{M}$ and $70 \mathrm{mg}(0.06 \mathrm{mmol}) \mathrm{Pd}\left(\mathrm{PPh}_{3}\right)_{4}$. The system was sealed under $\mathrm{Ar}$ and refluxed for 2 days at 85 ${ }^{\circ} \mathrm{C}$. After that period, the reaction mixture was extracted with THF which was then removed through rotary evaporation. The resulting solid was filtrated and copiously washed with water. The crude residue was purified through column chromatography on silica gel with petroleum ether/ethyl acetate mixture $(2: 1 \mathrm{v} / \mathrm{v})$ as an eluent, to give a grey -double substituted- precipitate.

Compound 2: A round bottom flask $(50 \mathrm{ml})$ was loaded with $0.4 \mathrm{~g}$ of 5-boronoisophthalic acid (1.905 $\mathrm{mmol}$ ), $20 \mathrm{ml}$ ethanol and $0.4 \mathrm{ml} \mathrm{c}$. $\mathrm{H}_{2} \mathrm{SO}_{4} 98 \%$. The mixture was refluxed for 12 hours. Ethanol was removed through rotary evaporation and then cold D.I. water was added to the flask until the precipitation of a white solid, which was isolated through filtration. 
Compound 3: In a dried under Ar double neck round bottom flask, $0.11 \mathrm{~g}$ of (1) $(0.258 \mathrm{mmol})$ were added along with $20 \mathrm{ml}$ dry THF, $0.103 \mathrm{~g}(0.388 \mathrm{mmol})$ of (2), $5 \mathrm{ml} \mathrm{Na} \mathrm{CO}_{3} 2 \mathrm{M}$ and $30 \mathrm{mg}(0.026$ $\mathrm{mmol}) \mathrm{Pd}\left(\mathrm{PPh}_{3}\right)_{4}$. The system was sealed under $\mathrm{Ar}$ and refluxed for 24 hours at $85^{\circ} \mathrm{C}$. After that period, the reaction mixture was dried under rotary evaporation and then extracted with ethyl acetate. The organic phase was dried over $\mathrm{MgSO}_{4}$, and the crude product was isolated after filtration and drying of the filtrate under vacuum. The crude residue was purified through column chromatography on silica gel with petroleum ether/ethyl acetate mixture $(4: 1 \mathrm{v} / \mathrm{v})$ as an eluent, to give a grey precipitate.

Compound 4: Compound $3(60 \mathrm{mg})$ was added to a round bottom flask with $3 \mathrm{ml} \mathrm{MeOH}, 3 \mathrm{ml}$ THF and $2 \mathrm{ml} \mathrm{NaOH} 5 \mathrm{~N}$. The mixture was refluxed at $85^{\circ} \mathrm{C}$ for 12 hours. After that period $15 \mathrm{ml}$ of $\mathrm{HCl} 3 \mathrm{~N}$ were added to the reaction mixture, producing an off-white solid which was filtrated and then copiously washed with water. Finally, the solid was dried under vacuum.

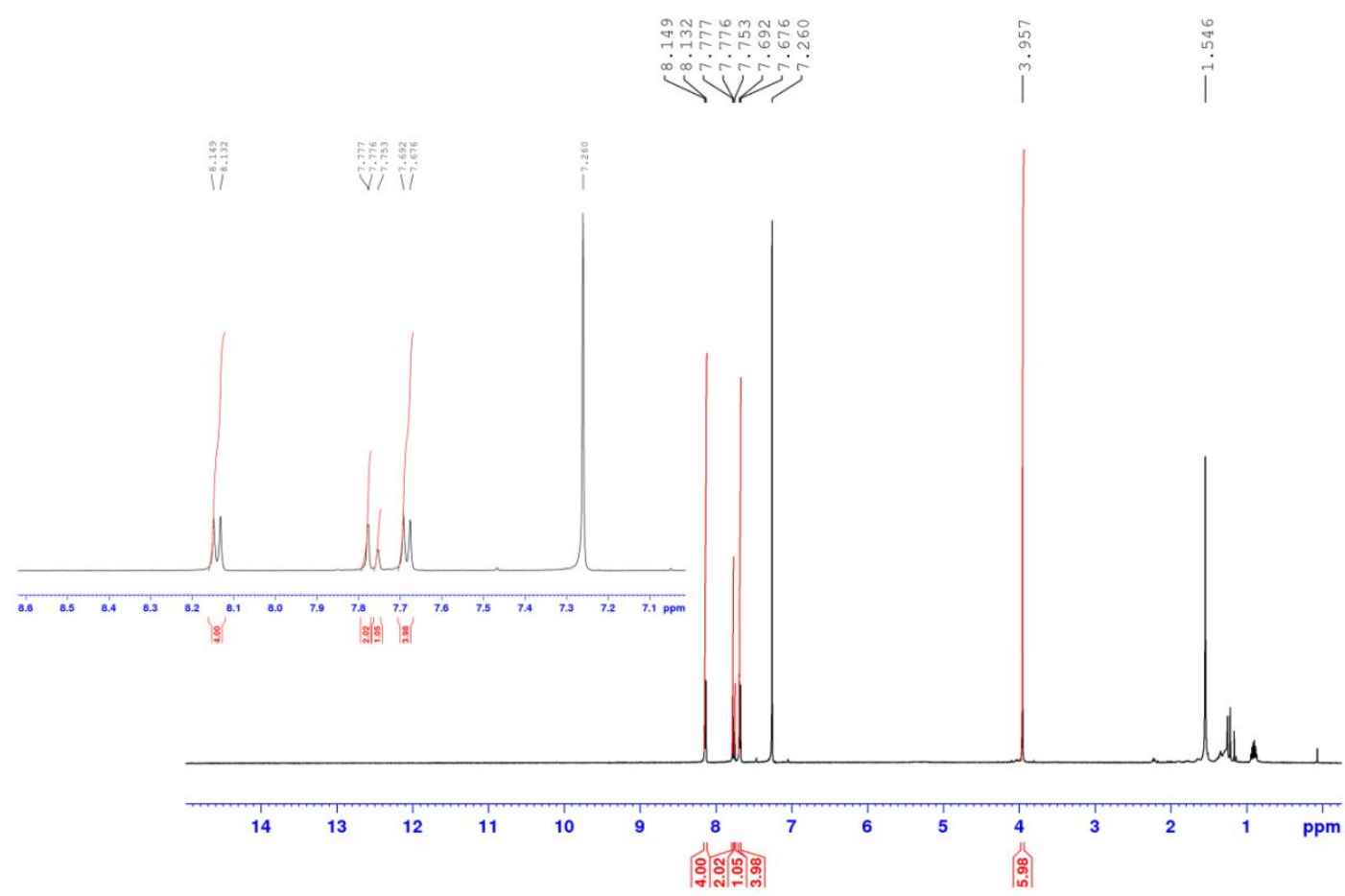

Figure S1. ${ }^{1} \mathrm{HNMR}$ spectrum of compound 1 in $\mathrm{CDCl}_{3}(500 \mathrm{MHz})$. 


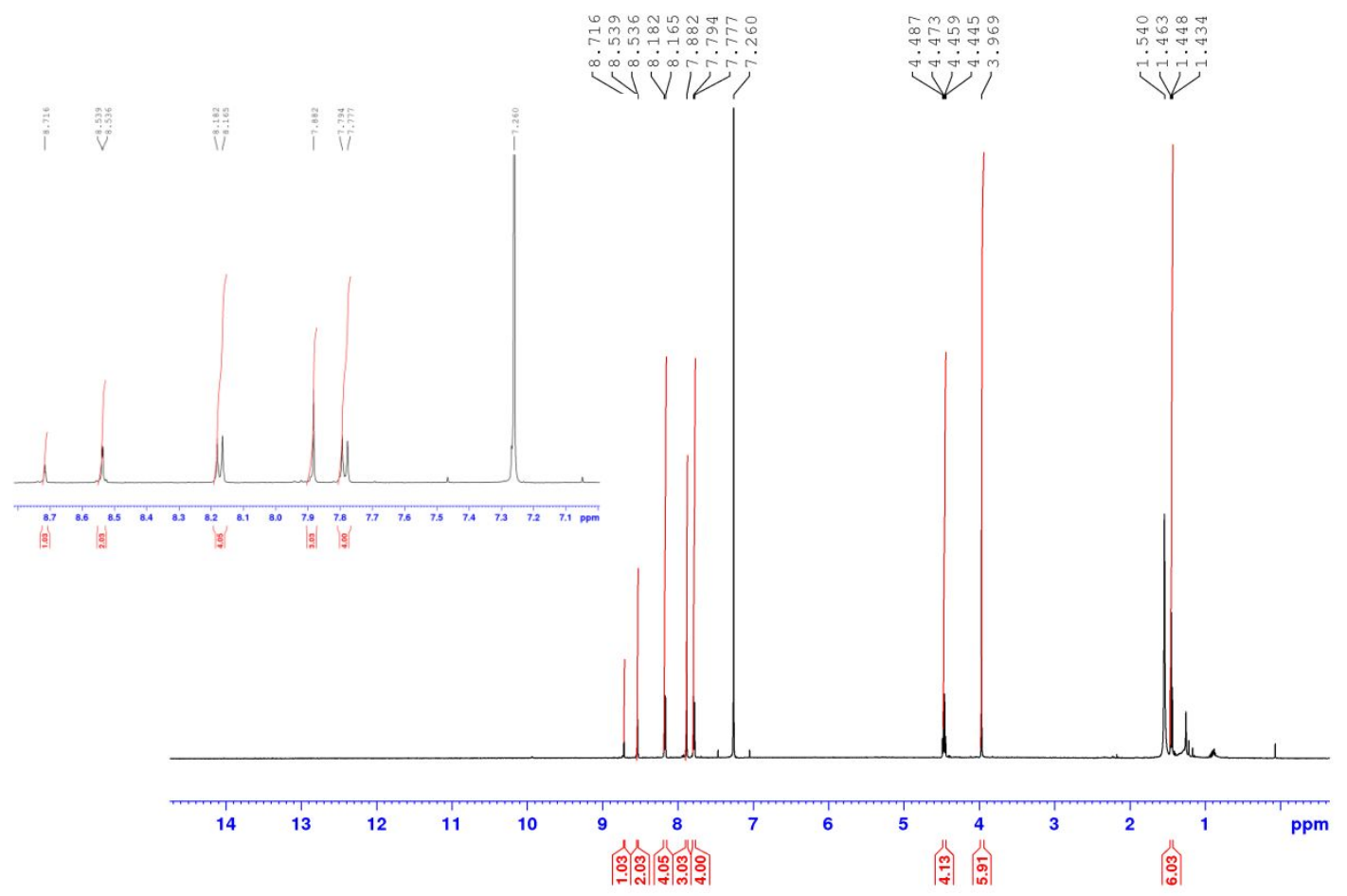

Figure S2. ${ }^{1} \mathrm{HNMR}$ spectrum of compound $\mathbf{3}$ in $\mathrm{CDCl}_{3}(500 \mathrm{MHz})$.

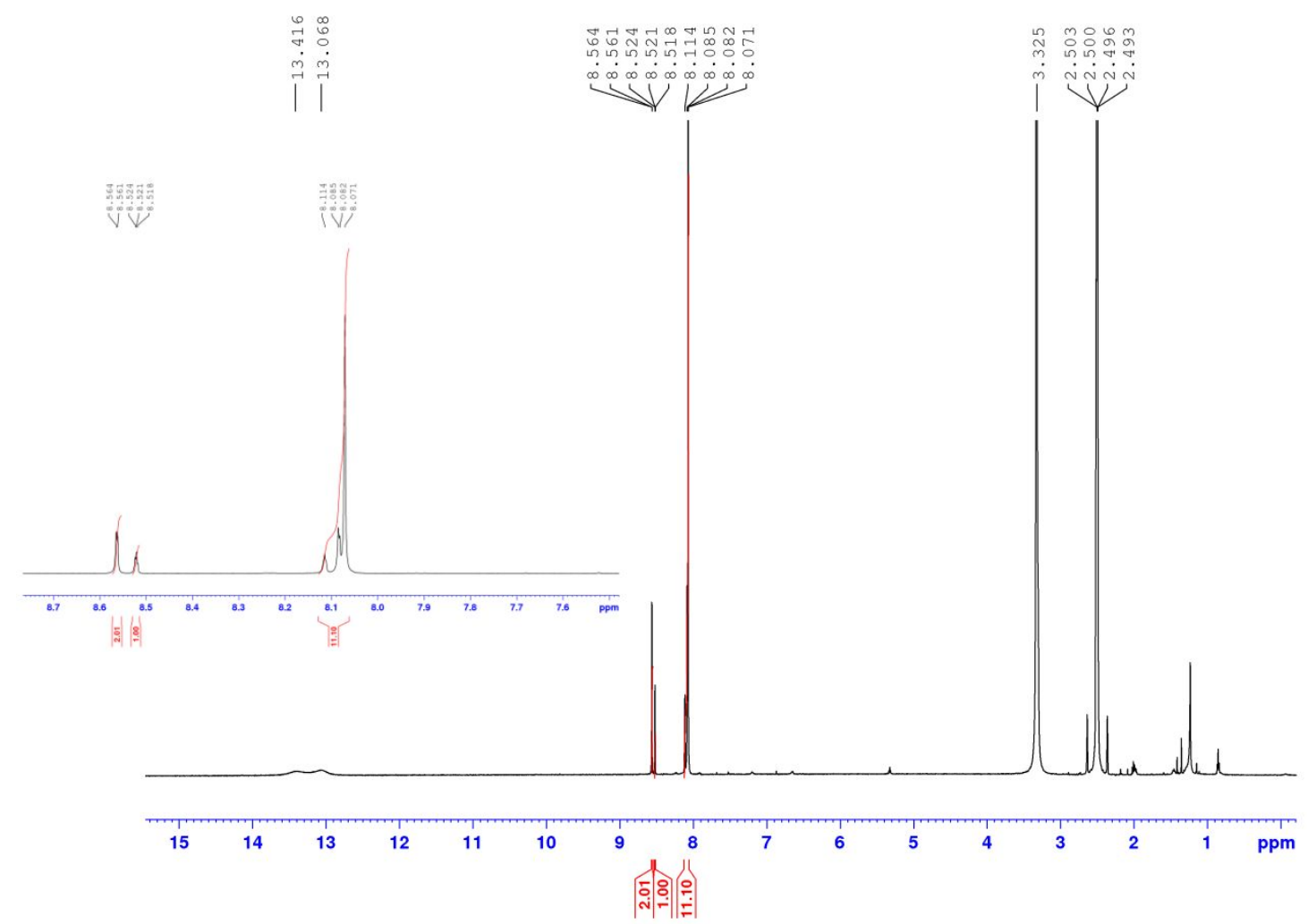

Figure S3. ${ }^{1} \mathrm{HNMR}$ spectrum of compound 4 in DMSO- $\mathrm{d}_{6}(500 \mathrm{MHz})$. 


\section{MOF synthesis}

Synthesis of $\mathrm{Y}$-thc-MOF-1. $\mathrm{Y}\left(\mathrm{NO}_{3}\right)_{3} \cdot 6 \mathrm{H}_{2} \mathrm{O}(13.7 \mathrm{mg}, 0.036 \mathrm{mmol}), 2$-fluorobenzoic acid $(200 \mathrm{mg}, 1.43$ $\mathrm{mmol}), \mathrm{H}_{2} \mathrm{O}(0.75 \mathrm{ml})$ and $\mathrm{H}_{4} \mathrm{~L}(4.3 \mathrm{mg}, 0.009 \mathrm{mmol})$ were added to a $3 \mathrm{ml}$ solution of DMF, which was then placed in a $20 \mathrm{ml}$ glass scintillation vial. The vial was sealed and placed in an isothermal oven at $11{ }^{\circ} \mathrm{C}$ for 24 hours. During this period colorless, large rhombic crystals were formed. (40\% yield based on $\mathrm{H}_{4} \mathrm{~L}$ ).

Synthesis of Sm-thc-MOF-1 $\mathrm{Sm}\left(\mathrm{NO}_{3}\right)_{3} \cdot 6 \mathrm{H}_{2} \mathrm{O}(16.0 \mathrm{mg}, 0.036 \mathrm{mmol}), 2$-fluorobenzoic acid $(200 \mathrm{mg}$, $1.43 \mathrm{mmol}), \mathrm{H}_{2} \mathrm{O}(0.75 \mathrm{ml})$ and $\mathrm{H}_{4} \mathrm{~L}(4.3 \mathrm{mg}, 0.009 \mathrm{mmol})$ were added to a $3 \mathrm{ml}$ solution of DMF, which was then placed in a $20 \mathrm{ml}$ glass scintillation vial. The vial was sealed and placed in an isothermal oven at $115{ }^{\circ} \mathrm{C}$ for 24 hours. During this period colorless, large rhombic crystals were formed. (30\% yield based on $\mathrm{H}_{4} \mathrm{~L}$ ).

Synthesis of Eu-thc-MOF-1. Eu( $\left(\mathrm{NO}_{3}\right)_{3} \cdot 6 \mathrm{H}_{2} \mathrm{O}(16.1 \mathrm{mg}, 0.036 \mathrm{mmol}), 2$-fluorobenzoic acid (100 mg, $0.71 \mathrm{mmol}), \mathrm{H}_{2} \mathrm{O}(0.75 \mathrm{ml})$ and $\mathrm{H}_{4} \mathrm{~L}(4.3 \mathrm{mg}, 0.009 \mathrm{mmol})$ were added to a $3 \mathrm{ml}$ solution of DMF, which was then placed in a $20 \mathrm{ml}$ glass scintillation vial. The vial was sealed and placed in an isothermal oven at $115{ }^{\circ} \mathrm{C}$ for 72 hours. During this period colorless, large rhombic crystals were formed. (25\% yield based on $\left.\mathrm{H}_{4} \mathrm{~L}\right)$.

Synthesis of Tb-thc-MOF-1. $\mathrm{Tb}\left(\mathrm{NO}_{3}\right)_{3} \cdot 6 \mathrm{H}_{2} \mathrm{O}(16.3 \mathrm{mg}, 0.036 \mathrm{mmol}), 2$-fluorobenzoic acid (100 mg, $0.71 \mathrm{mmol}), \mathrm{H}_{2} \mathrm{O}\left(0.75 \mathrm{ml}\right.$ ) and $\mathrm{H}_{4} \mathrm{~L}(4.3 \mathrm{mg}, 0.009 \mathrm{mmol})$ were added to a $3 \mathrm{ml}$ solution of DMF, which was then placed in a $20 \mathrm{ml}$ glass scintillation vial. The vial was sealed and placed in an isothermal oven at $115{ }^{\circ} \mathrm{C}$ for 48 hours. During this period colorless, large rhombic crystals were formed. (40\% yield based on $\left.\mathrm{H}_{4} \mathrm{~L}\right)$.

Synthesis of Dy-thc-MOF-1. Dy $\left(\mathrm{NO}_{3}\right)_{3} \cdot 5 \mathrm{H}_{2} \mathrm{O}(15.8 \mathrm{mg}, 0.036 \mathrm{mmol}), 2$-fluorobenzoic acid (100 mg, $0.71 \mathrm{mmol}), \mathrm{H}_{2} \mathrm{O}\left(0.75 \mathrm{ml}\right.$ ) and $\mathrm{H}_{4} \mathrm{~L}(4.3 \mathrm{mg}, 0.009 \mathrm{mmol})$ were added to a $3 \mathrm{ml}$ solution of DMF, which was then placed in a $20 \mathrm{ml}$ glass scintillation vial. The vial was sealed and placed in an isothermal oven at $115{ }^{\circ} \mathrm{C}$ for 48 hours. During this period colorless, large rhombic crystals were formed. (35\% yield based on $\left.\mathrm{H}_{4} \mathrm{~L}\right)$.

Synthesis of Ho-thc-MOF-1. $\mathrm{Ho}\left(\mathrm{NO}_{3}\right)_{3} \cdot 5 \mathrm{H}_{2} \mathrm{O}(15.9 \mathrm{mg}, 0.036 \mathrm{mmol}), 2$-fluorobenzoic acid $(200 \mathrm{mg}$, $1.43 \mathrm{mmol}), \mathrm{H}_{2} \mathrm{O}(0.375 \mathrm{ml})$ and $\mathrm{H}_{4} \mathrm{~L}(4.3 \mathrm{mg}, 0.009 \mathrm{mmol})$ were added to a $1.5 \mathrm{ml}$ solution of DMF, which was then placed in a $20 \mathrm{ml}$ glass scintillation vial. The vial was sealed and placed in an isothermal oven at $115{ }^{\circ} \mathrm{C}$ for 48 hours. During this period pink, small rhombic crystals were formed. ( $35 \%$ yield based on $\mathrm{H}_{4} \mathrm{~L}$ ).

Synthesis of Er-thc-MOF-1. $\mathrm{Er}\left(\mathrm{NO}_{3}\right)_{3} \cdot 5 \mathrm{H}_{2} \mathrm{O}(16.0 \mathrm{mg}, 0.036 \mathrm{mmol})$, 2-fluorobenzoic acid (200 mg, 1.43 mmol), $\mathrm{H}_{2} \mathrm{O}(0.375 \mathrm{ml})$ and $\mathrm{H}_{4} \mathrm{~L}(4.3 \mathrm{mg}, 0.009 \mathrm{mmol})$ were added to a $1.5 \mathrm{ml}$ solution of DMF, which was then placed in a $20 \mathrm{ml}$ glass scintillation vial. The vial was sealed and placed in an isothermal oven at $105^{\circ} \mathrm{C}$ for 48 hours. During this period pink, small rhombic crystals were formed. (30\% yield based on $\mathrm{H}_{4} \mathrm{~L}$ ).

\section{Post-synthetic exchange procedure}

The post-synthetic exchange of the coordinated 2-FBA with formate was achieved according to the following procedure: $10 \mathrm{mg}$ of as made material were immersed in $2.6 \mathrm{ml}$ of DMF in the presence of $0.1 \mathrm{ml} \mathrm{HCOOH}(95 \%)$. The mixture was heated overnight at $100{ }^{\circ} \mathrm{C}$. After this period the crystalline solid was copiously washed with fresh DMF and stored in DMF. The complete removal of 2-FBA was confirmed by ${ }^{1} \mathrm{H}$ NMR in an acid-digested sample (see Figure S35). 


\section{Scanning Electron Microscopy (SEM) Images}

Table S1. Scanning electron microscopy images of as-made RE-thc-MOF-1 crystals.

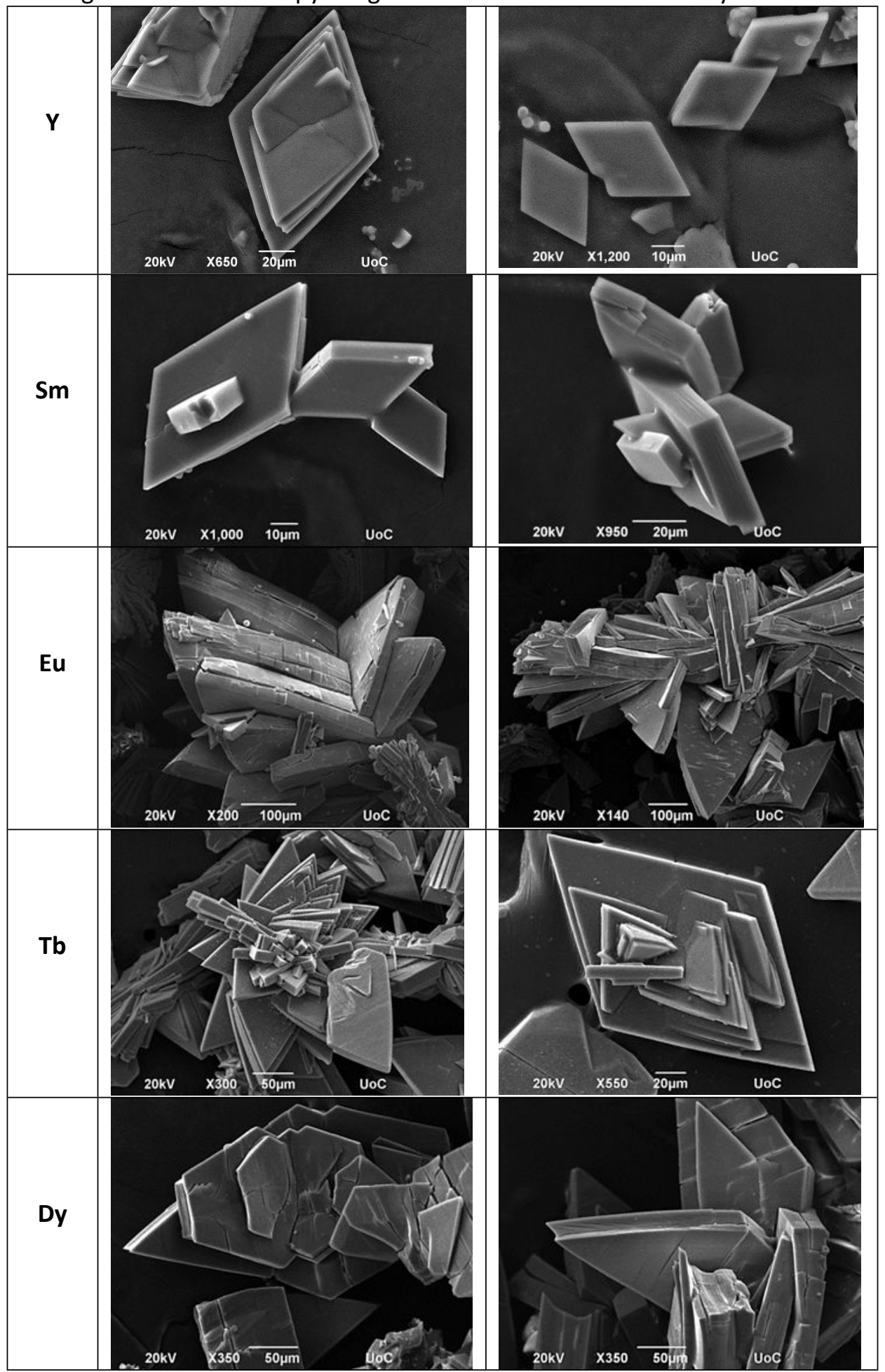



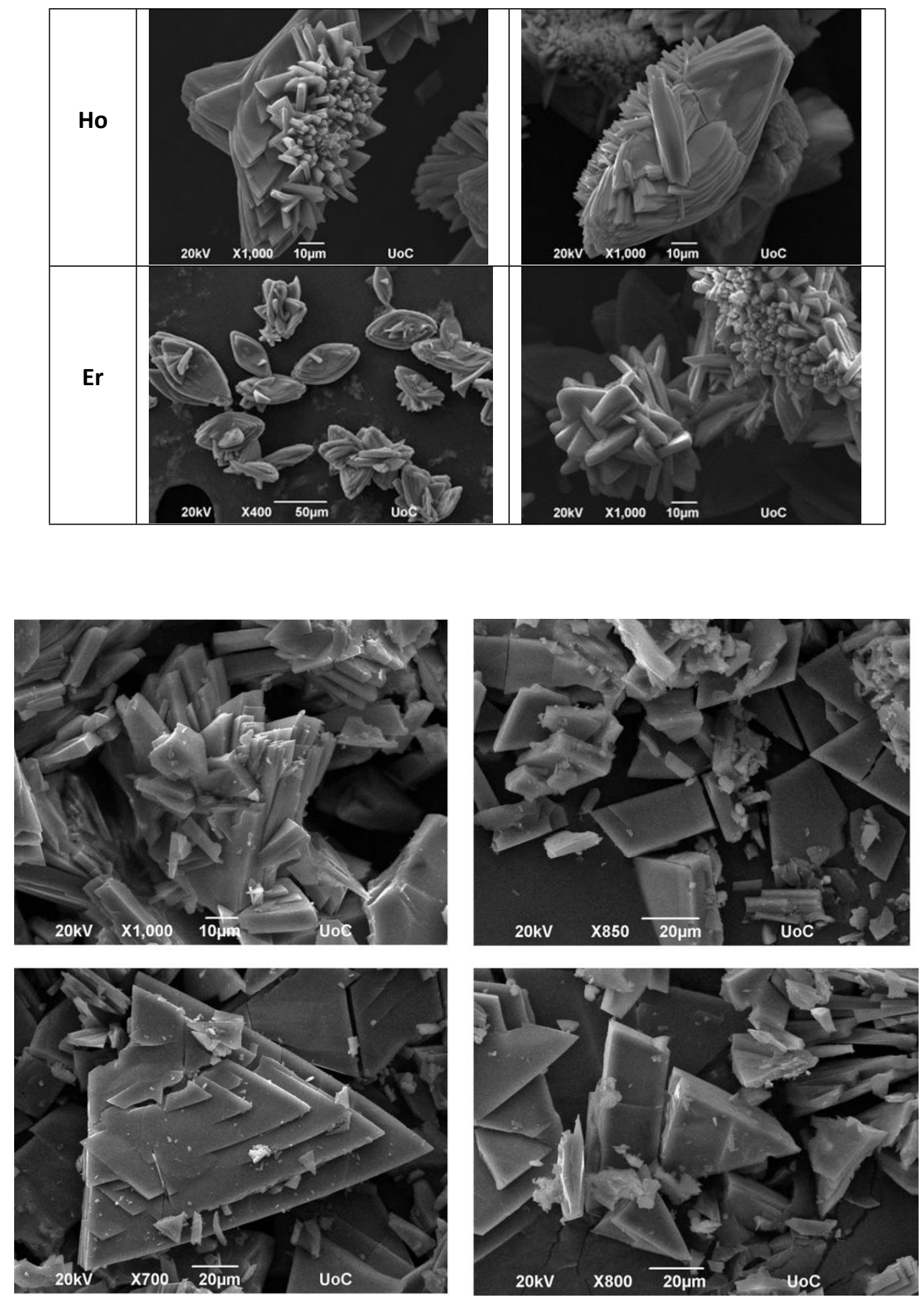

Figure S4. Representative SEM images of Y-thc-MOF-1 after gas sorption measurements. 

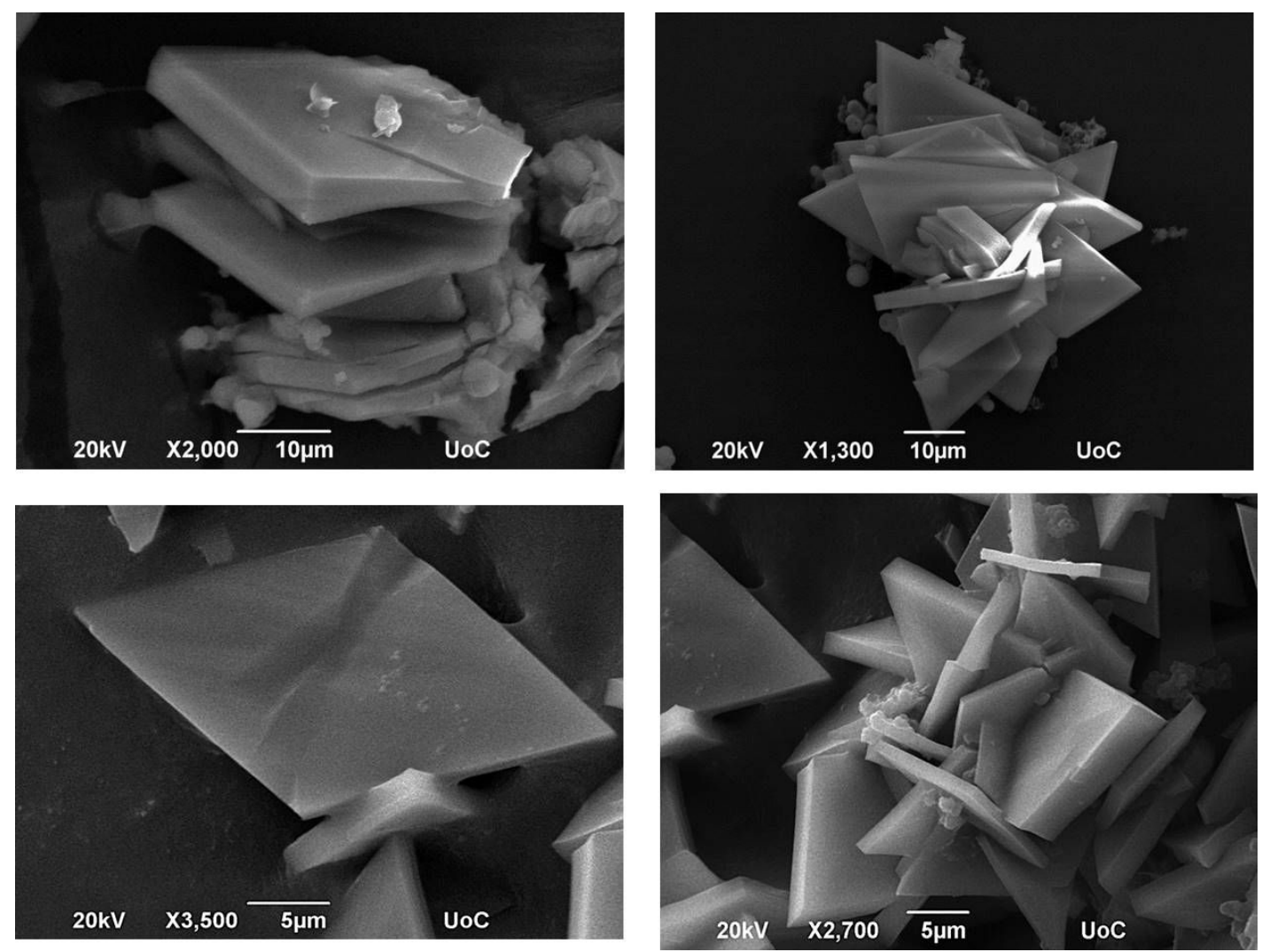

Figure S5. Representative SEM images of Y-thc-MOF-1 after vapor sorption measurements.

\section{Single crystal X-ray crystallography}

All structures were determined using OLEX2 ${ }^{5}$, solved using SHELXT ${ }^{6,7}$ and refined with SHELXL-2014 ${ }^{8}$. The structures exhibit relatively large voids filled with disordered solvent molecules and $\left[\mathrm{NH}_{2}\left(\mathrm{CH}_{3}\right)_{2}\right]^{2+}$ cation. In all cases, the $\left[\mathrm{RE}_{6}\left(\mu_{3}-\mathrm{OH}\right)_{8}\right]$ clusters as well as the ligand are well ordered and refined anisotropically, resulting in small displacement parameters. However, some of the coordinated 2-fluorobenzoate anions are found disordered and for this reason certain restraints/constraints in regards to distance (DFIX, SADI), geometry (AFIX 66), planarity (FLAT) and ADPs (EADP, ISOR, RIGU, SIMU) have been used in some structures. In some cases, we were unable to fully model all of the existing 2-fluorobenzoates, due to weak crystal diffraction and the large disorder especially in the areas of these moieties. In these cases, we have modelled the carboxyl group and the next connecting $C$ atom in order to demonstrate the directionality of the aromatic ring. In the case of Y-thc-MOF-1_EtOH_150K this was not possible to do; therefore, only the $\mathrm{O}$ atoms of the carboxyl group have been modelled. In all cases, the presence of 2-fluorobenzoates in the compounds was confirmed through ${ }^{1} \mathrm{H}$ NMR studies (see ${ }^{1} \mathrm{H}$ NMR section). The contribution of disordered counterions, unmodelled fluorobenzoates and solvent molecules was treated as diffuse using the solvent mask routine implemented in the OLEX2 interface. All non- $\mathrm{H}$ atoms were refined with anisotropic thermal parameters, and $\mathrm{H}$-atoms were introduced at calculated positions and allowed to ride on their carrier atoms. 
Table S2. Selected unit cell parameters for all RE-thc-MOF-1 frameworks. All compounds crystallize in the Pnma space group.

\begin{tabular}{lcccc}
\hline \multicolumn{1}{c}{ Compound } & $\mathrm{a}(\AA)$ & $\mathrm{b}(\AA)$ & $\mathrm{c}(\AA)$ & $\mathrm{V}\left(\AA^{3}\right)$ \\
\hline Y-thc-MOF-1_DMF_200K & $25.9045(8)$ & $32.3961(10)$ & $14.6910(5)$ & $12328.8(7)$ \\
Y-thc-MOF-1_EtOH_270K & $25.401(5)$ & $27.779(6)$ & $14.625(3)$ & $10320(4)$ \\
Y-thc-MOF-1_EtOH_280K & $25.3457(10)$ & $26.3162(12)$ & $14.3573(6)$ & $9576.4(7)$ \\
Y-thc-MOF-1_EtOH_200K & $25.3009(10)$ & $25.9126(12)$ & $14.3317(6)$ & $9396.0(7)$ \\
Y-thc-MOF-1_EtOH_150K & $25.2874(10)$ & $25.7770(13)$ & $14.3158(6)$ & $9331.5(7)$ \\
Y-thc-MOF-1_EtOH_100K 1 & 25.3 & 25.71 & 14.29 & 9295.12 \\
Y-thc-MOF-1_EtOH_280K & $25.2765(11)$ & $25.9792(13)$ & $14.3824(7)$ & $9444.4(8)$ \\
Y-thc-MOF-1_formate_DMF_220K & $25.004(3)$ & $27.689(3)$ & $14.0884(15)$ & $9753.9(19)$ \\
Y-thc-MOF-1_formate_EtOH_200K & $25.227(2)$ & $29.914(3)$ & $14.9725(12)$ & $11298.7(16)$ \\
Sm-thc-MOF-1_DMF_220K & $26.3158(8)$ & $32.6185(11)$ & $14.8574(5)$ & $12753.3(7)$ \\
Eu-thc-MOF-1_DMF_220K & $26.3338(9)$ & $32.6870(12)$ & $14.8669(5)$ & $12797.0(8)$ \\
Tb-thc-MOF-1_DMF_220K & $26.370(6)$ & $32.626(7)$ & $14.831(3)$ & $12760(5)$ \\
Tb-thc-MOF-1_EtOH_210K & $25.4696(18)$ & $28.104(2)$ & $14.5490(11)$ & $10414.3(13)$ \\
Dy-thc-MOF-1_DMF_210K & $25.9882(6)$ & $32.5499(8)$ & $14.7402(4)$ & $12468.9(5)$ \\
\hline
\end{tabular}

${ }^{1}$ Only partial data were collected at $100 \mathrm{~K}$ in order to determine the unit cell. Temperature was then returned to $280 \mathrm{~K}$ before full data were collected.

Table S3. Crystal data of Y-thc-MOF-1, as made in DMF, measured at $200 \mathrm{~K}$.

\begin{tabular}{|c|c|}
\hline Identification code & Y-thc-MOF-1_DMF_200K \\
\hline Empirical formula & $\mathrm{C}_{78} \mathrm{H}_{48} \mathrm{~F}_{3} \mathrm{O}_{36} \mathrm{Y}_{6}$ \\
\hline Formula weight & 2151.62 \\
\hline Temperature/K & 200.0 \\
\hline Crystal system & orthorhombic \\
\hline Space group & Pnma \\
\hline $\mathrm{a} / \AA ̊$ & $25.9045(8)$ \\
\hline $\mathrm{b} / \AA$ & $32.3961(10)$ \\
\hline$c / \AA ̊$ & $14.6910(5)$ \\
\hline$\alpha /^{\circ}$ & 90 \\
\hline$\beta /^{\circ}$ & 90 \\
\hline$\gamma /{ }^{\circ}$ & 90 \\
\hline Volume $/ \AA^{3}$ & $12328.8(7)$ \\
\hline Z & 4 \\
\hline$\rho_{\text {calc }} g / \mathrm{cm}^{3}$ & 1.159 \\
\hline$\mu / \mathrm{mm}^{1}$ & 4.194 \\
\hline$F(000)$ & 4260.0 \\
\hline Crystal size $/ \mathrm{mm}^{3}$ & $0.04 \times 0.04 \times 0.025$ \\
\hline Radiation & CuK $\alpha(\lambda=1.54178)$ \\
\hline $2 \Theta$ range for data collection $/^{\circ}$ & 6.606 to 100.88 \\
\hline Index ranges & $-25 \leq h \leq 25,-31 \leq k \leq 32,-14 \leq \mathrm{I} \leq 13$ \\
\hline Reflections collected & 45074 \\
\hline Independent reflections & $6577\left[R_{\text {int }}=0.0534, R_{\text {sigma }}=0.0345\right]$ \\
\hline Data/restraints/parameters & $6577 / 32 / 444$ \\
\hline Goodness-of-fit on $F^{2}$ & 1.072 \\
\hline Final $R$ indexes $[\mid>=2 \sigma(I)]$ & $\mathrm{R}_{1}=0.0858, \mathrm{wR}_{2}=0.2307$ \\
\hline
\end{tabular}


Table S4. Crystal data of Y-thc-MOF-1, immersed in EtOH, measured at $270 \mathrm{~K}$.

\begin{tabular}{|c|c|}
\hline Identification code & Y-thc-MOF-1_EtOH_270K \\
\hline Empirical formula & $\mathrm{C}_{63} \mathrm{H}_{37} \mathrm{O}_{32} \mathrm{Y}_{6}$ \\
\hline Formula weight & 1839.38 \\
\hline Temperature/K & 270.01 \\
\hline Crystal system & orthorhombic \\
\hline Space group & Pnma \\
\hline $\mathrm{a} / \AA ̊$ & $25.401(5)$ \\
\hline $\mathrm{b} / \AA ̊ A$ & $27.779(6)$ \\
\hline$c / \AA ̊$ & $14.625(3)$ \\
\hline$\alpha /^{\circ}$ & 90 \\
\hline$\beta /^{\circ}$ & 90 \\
\hline$\gamma /{ }^{\circ}$ & 90 \\
\hline Volume $/ \AA^{3}$ & $10320(4)$ \\
\hline $\mathrm{Z}$ & 4 \\
\hline$\rho_{\text {calc }} \mathrm{g} / \mathrm{cm}^{3}$ & 1.184 \\
\hline$\mu / \mathrm{mm}^{1}$ & 4.854 \\
\hline$F(000)$ & 3620.0 \\
\hline Crystal size $/ \mathrm{mm}^{3}$ & $0.04 \times 0.04 \times 0.02$ \\
\hline Radiation & CuK $\alpha(\lambda=1.54178)$ \\
\hline $2 \Theta$ range for data collection $/^{\circ}$ & 6.364 to 101.032 \\
\hline Index ranges & $-25 \leq h \leq 18,-27 \leq k \leq 27,-14 \leq \mathrm{I} \leq 12$ \\
\hline Reflections collected & 23653 \\
\hline Independent reflections & $5505\left[R_{\text {int }}=0.0654, R_{\text {sigma }}=0.0637\right]$ \\
\hline Data/restraints/parameters & $5505 / 24 / 265$ \\
\hline Goodness-of-fit on $F^{2}$ & 1.083 \\
\hline Final $R$ indexes $[I>=2 \sigma(I)]$ & $\mathrm{R}_{1}=0.1097, \mathrm{wR}_{2}=0.3138$ \\
\hline Final $R$ indexes [all data] & $\mathrm{R}_{1}=0.1358, w \mathrm{R}_{2}=0.3378$ \\
\hline Largest diff. peak/hole / e $\AA^{-3}$ & $1.16 /-1.28$ \\
\hline
\end{tabular}

Table S5. Crystal data of Y-thc-MOF-1, immersed in EtOH, measured at $280 \mathrm{~K}$.

\begin{tabular}{lc}
\hline Identification code & Y-thc-MOF-1_EtOH_280K \\
\hline Empirical formula & $\mathrm{C}_{67} \mathrm{H}_{39} \mathrm{FO}_{32} \mathrm{Y}_{6}$ \\
Formula weight & 1908.44 \\
Temperature/K & 279.99 \\
Crystal system & orthorhombic \\
Space group & Pnma \\
a/A & $25.3457(10)$ \\
b/A & $26.3162(12)$ \\
$c / \AA$ & $14.3573(6)$ \\
$\alpha /{ }^{\circ}$ & 90 \\
$\beta /{ }^{\circ}$ & 90
\end{tabular}


$\gamma /{ }^{\circ}$

Volume/ $/ \AA^{3}$

Z

$\rho_{\text {calc }} \mathrm{g} / \mathrm{cm}^{3}$

$\mu / \mathrm{mm}^{1}$

$\mathrm{F}(000)$

Crystal size $/ \mathrm{mm}^{3}$

Radiation

$2 \Theta$ range for data collection ${ }^{\circ}$

Index ranges

Reflections collected

Independent reflections

Data/restraints/parameters

Goodness-of-fit on $\mathrm{F}^{2}$

Final $R$ indexes $[\mid>=2 \sigma(I)]$

Final $R$ indexes [all data]

Largest diff. peak/hole / e $\AA^{-3}$
90

$9576.4(7)$

4

1.324

5.266

3760.0

$0.04 \times 0.04 \times 0.025$

$\operatorname{CuK} \alpha(\lambda=1.54178)$

6.718 to 101.276

$-24 \leq \mathrm{h} \leq 25,-26 \leq \mathrm{k} \leq 20,-11 \leq \mathrm{I} \leq 14$

25847

$5160\left[R_{\text {int }}=0.0853, R_{\text {sigma }}=0.0697\right]$

$5160 / 17 / 296$

1.362

$\mathrm{R}_{1}=0.1237, \mathrm{wR}_{2}=0.3584$

$R_{1}=0.1538, w R_{2}=0.3844$

$1.64 /-1.71$

Table S6. Crystal data of Y-thc-MOF-1, immersed in EtOH, measured at $200 \mathrm{~K}$.

\begin{tabular}{|c|c|}
\hline Identification code & Y-thc-MOF-1_EtOH_200K \\
\hline Empirical formula & $\mathrm{C}_{68} \mathrm{H}_{37} \mathrm{FO}_{32} \mathrm{Y}_{6}$ \\
\hline Formula weight & 1918.43 \\
\hline Temperature/K & 199.99 \\
\hline Crystal system & orthorhombic \\
\hline Space group & Pnma \\
\hline$a / \AA ̊$ & $25.3009(10)$ \\
\hline $\mathrm{b} / \AA ̊ \AA$ & $25.9126(12)$ \\
\hline$c / \AA ̊$ & $14.3317(6)$ \\
\hline$\alpha /^{\circ}$ & 90 \\
\hline$\beta /{ }^{\circ}$ & 90 \\
\hline$\gamma /{ }^{\circ}$ & 90 \\
\hline Volume $/ \AA^{3}$ & $9396.0(7)$ \\
\hline Z & 4 \\
\hline$\rho_{\text {calc }} \mathrm{g} / \mathrm{cm}^{3}$ & 1.356 \\
\hline$\mu / \mathrm{mm}^{1}$ & 5.371 \\
\hline$F(000)$ & 3776.0 \\
\hline Crystal size $/ \mathrm{mm}^{3}$ & $0.04 \times 0.04 \times 0.025$ \\
\hline Radiation & $\operatorname{CuK} \alpha(\lambda=1.54178)$ \\
\hline $2 \Theta$ range for data collection $/^{\circ}$ & 6.822 to 100.992 \\
\hline Index ranges & $-25 \leq h \leq 24,-20 \leq k \leq 25,-11 \leq \mathrm{I} \leq 14$ \\
\hline Reflections collected & 28068 \\
\hline Independent reflections & $5039\left[R_{\text {int }}=0.0729, R_{\text {sigma }}=0.0535\right]$ \\
\hline Data/restraints/parameters & $5039 / 17 / 262$ \\
\hline Goodness-of-fit on $F^{2}$ & 1.060 \\
\hline Final $R$ indexes $[\mid>=2 \sigma(I)]$ & $\mathrm{R}_{1}=0.1092, \mathrm{wR}_{2}=0.3078$ \\
\hline Final R indexes [all data] & $\mathrm{R}_{1}=0.1350, \mathrm{wR}_{2}=0.3276$ \\
\hline
\end{tabular}


Table S7. Crystal data of Y-thc-MOF-1, immersed in EtOH, measured at $150 \mathrm{~K}$.

\begin{tabular}{|c|c|}
\hline Identification code & Y-thc-MOF-1_EtOH_150K \\
\hline Empirical formula & $\mathrm{C}_{64} \mathrm{H}_{40} \mathrm{FO}_{32} \mathrm{Y}_{6}$ \\
\hline Formula weight & 1873.42 \\
\hline Temperature/K & 149.98 \\
\hline Crystal system & orthorhombic \\
\hline Space group & Pnma \\
\hline$a / \AA$ & $25.2874(10)$ \\
\hline$b / \AA$ & $25.7770(13)$ \\
\hline$c / \AA ̊$ & $14.3158(6)$ \\
\hline$\alpha /^{\circ}$ & 90 \\
\hline$\beta /^{\circ}$ & 90 \\
\hline$\gamma /{ }^{\circ}$ & 90 \\
\hline Volume/Å ${ }^{3}$ & $9331.5(7)$ \\
\hline Z & 4 \\
\hline$\rho_{\text {calc }} \mathrm{g} / \mathrm{cm}^{3}$ & 1.333 \\
\hline$\mu / \mathrm{mm}^{1}$ & 5.393 \\
\hline$F(000)$ & 3692.0 \\
\hline Crystal size $/ \mathrm{mm}^{3}$ & $0.04 \times 0.04 \times 0.025$ \\
\hline Radiation & $\operatorname{CuK} \alpha(\lambda=1.54178)$ \\
\hline $2 \Theta$ range for data collection $/^{\circ}$ & 6.858 to 100.992 \\
\hline Index ranges & $-25 \leq h \leq 23,-25 \leq k \leq 25,-14 \leq \mathrm{I} \leq 11$ \\
\hline Reflections collected & 28303 \\
\hline Independent reflections & $5003\left[R_{\text {int }}=0.0797, R_{\text {sigma }}=0.0608\right]$ \\
\hline Data/restraints/parameters & $5003 / 17 / 349$ \\
\hline Goodness-of-fit on $F^{2}$ & 1.074 \\
\hline Final $R$ indexes $[\mid>=2 \sigma(I)]$ & $\mathrm{R}_{1}=0.1025, \mathrm{wR}_{2}=0.2967$ \\
\hline Final R indexes [all data] & $\mathrm{R}_{1}=0.1353, \mathrm{wR}_{2}=0.3240$ \\
\hline Largest diff. peak/hole / e $\AA^{-3}$ & $1.15 /-1.56$ \\
\hline
\end{tabular}

Table S8. Crystal data of Y-thc-MOF-1, immersed in EtOH, with the crystal left at $100 \mathrm{~K}$ then measured at $280 \mathrm{~K}$.

\begin{tabular}{lc}
\hline Identification code & Y-thc-MOF-1_EtOH_280K_return_from_100K \\
\hline Empirical formula & $\mathrm{C}_{66} \mathrm{H}_{31} \mathrm{FO}_{32} \mathrm{Y}_{6}$ \\
Formula weight & 1888.37 \\
Temperature/K & 280.01 \\
Crystal system & orthorhombic \\
Space group & Pnma \\
a/A & $25.2765(11)$ \\
$\mathrm{b} / \AA$ & $25.9792(13)$ \\
$c / \AA$ & $14.3824(7)$ \\
$\alpha{ }^{\circ}$ & 90 \\
$\beta /{ }^{\circ}$ & 90 \\
$\gamma /{ }^{\circ}$ & 90
\end{tabular}


Volume/ $\AA^{3}$

Z

$\rho_{\text {calc }} \mathrm{g} / \mathrm{cm}^{3}$

$\mu / \mathrm{mm}^{1}$

$\mathrm{F}(000)$

Crystal size $/ \mathrm{mm}^{3}$

Radiation

$2 \Theta$ range for data collection $/^{\circ}$

Index ranges

Reflections collected

Independent reflections

Data/restraints/parameters

Goodness-of-fit on $\mathrm{F}^{2}$

Final $R$ indexes $[1>=2 \sigma(I)]$

Final $\mathrm{R}$ indexes [all data]

Largest diff. peak/hole / e $\AA^{-3}$
$9444.4(8)$

1.328

5.336

3704.0

$0.04 \times 0.04 \times 0.025$

CuK $\alpha(\lambda=1.54178)$

6.804 to 101.202

$-25 \leq h \leq 24,-26 \leq k \leq 20,-14 \leq \mathrm{I} \leq 11$

29338

$5090\left[R_{\text {int }}=0.0754, R_{\text {sigma }}=0.0561\right]$

$5090 / 23 / 262$

1.352

$R_{1}=0.1166, w R_{2}=0.3468$

$R_{1}=0.1460, w_{2}=0.3727$

$0.84 /-1.86$

Table 59. Crystal data of Y-thc-MOF-1, after post-synthetic exchange of 2-fluorobenzoates with formates, measured at $220 \mathrm{~K}$.

\begin{tabular}{|c|c|}
\hline Identification code & Y-thc-MOF-1_formate_exchange_220K \\
\hline Empirical formula & $\mathrm{C}_{29} \mathrm{H}_{22} \mathrm{O}_{16} \mathrm{Y}_{3}$ \\
\hline Formula weight & 893.19 \\
\hline Temperature/K & 219.99 \\
\hline Crystal system & orthorhombic \\
\hline Space group & Pnma \\
\hline $\mathrm{a} / \AA ̊$ & $25.004(3)$ \\
\hline $\mathrm{b} / \AA ̊$ & $27.689(3)$ \\
\hline$c / \AA ̊$ & $14.0884(15)$ \\
\hline$\alpha /^{\circ}$ & 90 \\
\hline$\beta /^{\circ}$ & 90 \\
\hline$\gamma /{ }^{\circ}$ & 90 \\
\hline Volume/Å ${ }^{3}$ & $9753.9(19)$ \\
\hline Z & 8 \\
\hline$\rho_{\text {calc }} g / \mathrm{cm}^{3}$ & 1.216 \\
\hline$\mu / \mathrm{mm}^{1}$ & 5.117 \\
\hline$F(000)$ & 3528.0 \\
\hline Crystal size $/ \mathrm{mm}^{3}$ & $0.03 \times 0.02 \times 0.02$ \\
\hline Radiation & CuK $\alpha(\lambda=1.54178)$ \\
\hline $2 \Theta$ range for data collection $/^{\circ}$ & 6.384 to 101.632 \\
\hline Index ranges & $-24 \leq \mathrm{h} \leq 25,-27 \leq \mathrm{k} \leq 27,-12 \leq \mathrm{I} \leq 14$ \\
\hline Reflections collected & 33827 \\
\hline Independent reflections & $5254\left[R_{\text {int }}=0.1611, R_{\text {sigma }}=0.1095\right]$ \\
\hline Data/restraints/parameters & $5254 / 12 / 300$ \\
\hline Goodness-of-fit on $F^{2}$ & 0.937 \\
\hline Final $R$ indexes $[\mid>=2 \sigma(I)]$ & $\mathrm{R}_{1}=0.1046, \mathrm{wR}_{2}=0.2810$ \\
\hline Final R indexes [all data] & $R_{1}=0.1676, w R_{2}=0.3428$ \\
\hline
\end{tabular}


Table S10. Crystal data of Sm-thc-MOF-1, as made in DMF, measured at $220 \mathrm{~K}$.

\begin{tabular}{|c|c|}
\hline Identification code & Sm-thc-MOF-1_DMF_220K \\
\hline Empirical formula & $\mathrm{C}_{68} \mathrm{H}_{40} \mathrm{FO}_{36} \mathrm{Sm}_{6}$ \\
\hline Formula weight & 2354.10 \\
\hline Temperature/K & 220.0 \\
\hline Crystal system & orthorhombic \\
\hline Space group & Pnma \\
\hline $\mathrm{a} / \AA ̊$ & $26.3158(8)$ \\
\hline$b / \AA ̊$ & $32.6185(11)$ \\
\hline$c / \AA$ & $14.8574(5)$ \\
\hline$\alpha /^{\circ}$ & 90 \\
\hline$\beta /^{\circ}$ & 90 \\
\hline$\gamma /{ }^{\circ}$ & 90 \\
\hline Volume $/ \AA^{3}$ & $12753.3(7)$ \\
\hline Z & 4 \\
\hline$\rho_{\text {calc }} \mathrm{g} / \mathrm{cm}^{3}$ & 1.226 \\
\hline$\mu / \mathrm{mm}^{1}$ & 20.876 \\
\hline$F(000)$ & 4468.0 \\
\hline Crystal size $/ \mathrm{mm}^{3}$ & $0.04 \times 0.025 \times 0.025$ \\
\hline Radiation & CuK $\alpha(\lambda=1.54178)$ \\
\hline $2 \Theta$ range for data collection $/^{\circ}$ & 5.418 to 118.212 \\
\hline Index ranges & $-29 \leq h \leq 29,-35 \leq k \leq 36,-15 \leq \mathrm{I} \leq 16$ \\
\hline Reflections collected & 55030 \\
\hline Independent reflections & $9378[$ Rint $=0.0910$, Rsigma $=0.0694]$ \\
\hline Data/restraints/parameters & $9378 / 48 / 396$ \\
\hline Goodness-of-fit on $F^{2}$ & 1.063 \\
\hline Final $R$ indexes $[I>=2 \sigma(I)]$ & $\mathrm{R} 1=0.0808, \mathrm{wR} 2=0.2351$ \\
\hline Final $R$ indexes [all data] & $\mathrm{R} 1=0.0953, \mathrm{wR} 2=0.2449$ \\
\hline Largest diff. peak/hole / e $\AA^{-3}$ & $1.89 /-2.92$ \\
\hline
\end{tabular}

Table S11. Crystal data of Eu-thc-MOF-1, as made in DMF, measured at $220 \mathrm{~K}$.

\begin{tabular}{lc}
\hline Identification code & Eu-thc-MOF-1_DMF_220K \\
\hline Empirical formula & $\mathrm{C}_{73} \mathrm{H}_{43} \mathrm{Eu}_{6} \mathrm{~F}_{2} \mathrm{O}_{36}$ \\
Formula weight & 2445.83 \\
Temperature/K & 220.0 \\
Crystal system & orthorhombic \\
Space group & Pnma \\
a/A & $26.3338(9)$ \\
b/A & $32.6870(12)$ \\
$c / \AA$ & $14.8669(5)$ \\
$\alpha /{ }^{\circ}$ & 90 \\
$\beta /{ }^{\circ}$ & 90 \\
$\gamma /{ }^{\circ}$ & 90
\end{tabular}


Volume $/ \AA^{3}$

Z

$\rho_{\text {calc }} \mathrm{g} / \mathrm{cm}^{3}$

$\mu / \mathrm{mm}^{1}$

$\mathrm{F}(000)$

Crystal size $/ \mathrm{mm}^{3}$

Radiation

$2 \Theta$ range for data collection $/{ }^{\circ}$

Index ranges

Reflections collected

Independent reflections

Data/restraints/parameters

Goodness-of-fit on $\mathrm{F}^{2}$

Final $R$ indexes $[1>=2 \sigma(I)]$

Final $R$ indexes [all data]

Largest diff. peak/hole / e $\AA^{-3}$
$12797.0(8)$

4

1.269

21.209

4660.0

$0.04 \times 0.025 \times 0.025$

CuK $\alpha(\lambda=1.54178)$

6.712 to 130.586

$-14 \leq h \leq 30,-37 \leq k \leq 38,-17 \leq \mathrm{l} \leq 17$

49390

$11004\left[R_{\text {int }}=0.0478, R_{\text {sigma }}=0.0416\right]$

$11004 / 6 / 331$

1.099

$\mathrm{R}_{1}=0.0650, \mathrm{wR}_{2}=0.1830$

$\mathrm{R}_{1}=0.0705, \mathrm{wR}_{2}=0.1885$

$3.78 /-2.56$

Table S12. Crystal data of Tb-thc-MOF-1, as made in DMF, measured at $220 \mathrm{~K}$.

Identification code

Empirical formula

Formula weight

Temperature/K

Crystal system

Space group

$\mathrm{a} / \AA ̊ \AA$

$\mathrm{b} / \AA ̊$

$c / \AA$

$\alpha /^{\circ}$

$\beta /^{\circ}$

$\gamma /{ }^{\circ}$

Volume/ $/ \AA^{3}$

Z

$\rho_{\text {calc }} \mathrm{g} / \mathrm{cm}^{3}$

$\mu / \mathrm{mm}^{1}$

$\mathrm{F}(000)$

Crystal size $/ \mathrm{mm}^{3}$

Radiation

$2 \Theta$ range for data collection/ ${ }^{\circ}$

Index ranges

Reflections collected

Independent reflections

Data/restraints/parameters

Goodness-of-fit on $\mathrm{F}^{2}$

Final $R$ indexes $[1>=2 \sigma(I)]$

Final $\mathrm{R}$ indexes [all data]

Largest diff. peak/hole / e $\AA^{-3}$
Tb-thc-MOF-1_DMF_220K

$\mathrm{C}_{63} \mathrm{H}_{36} \mathrm{O}_{36} \mathrm{~Tb}_{6}$
2322.44
219.99

orthorhombic

Pnma

26.370(6)

$32.626(7)$

$14.831(3)$

90

90

90

12760(5)

4

1.209

16.453

4368.0

$0.04 \times 0.025 \times 0.025$

CuK $\alpha(\lambda=1.54178)$

5.418 to 117.85

$-29 \leq h \leq 26,-36 \leq k \leq 35,-16 \leq \mathrm{I} \leq 16$

43233

$9222\left[R_{\text {int }}=0.0757, R_{\text {sigma }}=0.0676\right]$

$9222 / 8 / 355$

1.155

$\mathrm{R}_{1}=0.0879, \mathrm{wR}_{2}=0.2618$

$R_{1}=0.1050, w R_{2}=0.2777$

$2.59 /-2.13$ 
Table S13. Crystal data of Tb-thc-MOF-1, immersed in EtOH, measured at $210 \mathrm{~K}$.

\begin{tabular}{|c|c|}
\hline Identification code & Tb-thc-MOF-1_EtOH_210K \\
\hline Empirical formula & $\mathrm{C}_{86} \mathrm{H}_{45} \mathrm{~F}_{3} \mathrm{O}_{36} \mathrm{~Tb}_{6}$ \\
\hline Formula weight & 2664.74 \\
\hline Temperature/K & 210.0 \\
\hline Crystal system & orthorhombic \\
\hline Space group & Pnma \\
\hline $\mathrm{a} / \AA ̊$ & $25.4696(18)$ \\
\hline$b / A ̊$ & $28.104(2)$ \\
\hline$c / \AA ̊$ & $14.5490(11)$ \\
\hline$\alpha /^{\circ}$ & 90 \\
\hline$\beta /^{\circ}$ & 90 \\
\hline$\gamma /{ }^{\circ}$ & 90 \\
\hline Volume/Å3 & 10414.3(13) \\
\hline Z & 4 \\
\hline$\rho_{\text {calc }} \mathrm{g} / \mathrm{cm}^{3}$ & 1.700 \\
\hline$\mu / \mathrm{mm}^{1}$ & 20.295 \\
\hline$F(000)$ & 5064.0 \\
\hline Crystal size $/ \mathrm{mm}^{3}$ & $0.02 \times 0.015 \times 0.015$ \\
\hline Radiation & $\operatorname{CuK} \alpha(\lambda=1.54178)$ \\
\hline $2 \Theta$ range for data collection $/^{\circ}$ & 6.29 to 100.998 \\
\hline Index ranges & $-21 \leq h \leq 25,-28 \leq k \leq 28,-11 \leq \mathrm{I} \leq 14$ \\
\hline Reflections collected & 27544 \\
\hline Independent reflections & $5578\left[R_{\text {int }}=0.0776, R_{\text {sigma }}=0.0604\right]$ \\
\hline Data/restraints/parameters & $5578 / 74 / 293$ \\
\hline Goodness-of-fit on $F^{2}$ & 1.076 \\
\hline Final $R$ indexes $[\mid>=2 \sigma(I)]$ & $\mathrm{R}_{1}=0.0906, \mathrm{wR}_{2}=0.2541$ \\
\hline Final $R$ indexes [all data] & $\mathrm{R}_{1}=0.1232, \mathrm{wR}_{2}=0.2774$ \\
\hline Largest diff. peak/hole / e $\AA^{-3}$ & $1.85 /-1.20$ \\
\hline
\end{tabular}

Table S14. Crystal data of Dy-thc-MOF-1, as made in DMF, measured at $210 \mathrm{~K}$.

\begin{tabular}{lc}
\hline Identification code & Dy-thc-MOF-1_DMF_210K \\
\hline Empirical formula & $\mathrm{C}_{78} \mathrm{H}_{48} \mathrm{Dy}_{6} \mathrm{~F}_{3} \mathrm{O}_{36}$ \\
Formula weight & 2593.16 \\
Temperature/K & 210.0 \\
Crystal system & orthorhombic \\
Space group & Pnma \\
a/Å & $25.9882(6)$ \\
b/A & $32.5499(8)$ \\
$c / \AA$ & $14.7402(4)$ \\
$\alpha /^{\circ}$ & 90 \\
$\beta /{ }^{\circ}$ & 90 \\
$\gamma^{\circ}$ & 90 \\
Volume/Å & \\
$Z$ & $12468.9(5)$
\end{tabular}


$\rho_{\text {calc }} \mathrm{g} / \mathrm{cm}^{3}$

1.381

$\mu / \mathrm{mm}^{1}$

$\mathrm{F}(000)$

Crystal size $/ \mathrm{mm}^{3}$

Radiation

$2 \Theta$ range for data collection/ ${ }^{\circ}$

Index ranges

Reflections collected

Independent reflections

Data/restraints/parameters

Goodness-of-fit on $\mathrm{F}^{2}$

Final $R$ indexes $[1>=2 \sigma(I)]$

Final $\mathrm{R}$ indexes [all data]

Largest diff. peak/hole / e $\AA^{-3}$
19.430

4908.0

$0.03 \times 0.02 \times 0.015$

CuKa $(\lambda=1.54178)$

6.582 to 100.884

$-24 \leq h \leq 25,-29 \leq k \leq 32,-14 \leq \mathrm{l} \leq 14$

38954

$6660\left[R_{\text {int }}=0.0452, R_{\text {sigma }}=0.0300\right]$

$6660 / 41 / 308$

1.080

$R_{1}=0.0724, w R_{2}=0.2056$

$R_{1}=0.0797, w R_{2}=0.2136$

$5.06 /-2.58$

Table S15. Crystal data of Y-thc-MOF-1, immersed in EtOH after post-synthetic exchange of 2fluorobenzoates with formates, measured at $200 \mathrm{~K}$.

\begin{tabular}{|c|c|}
\hline Identification code & Y-thc-MOF-1_formate_EtOH_200K \\
\hline Empirical formula & $\mathrm{C}_{66} \mathrm{H}_{62} \mathrm{O}_{37} \mathrm{Y}_{6}$ \\
\hline Formula weight & 1980.61 \\
\hline Temperature/K & 200.0 \\
\hline Crystal system & orthorhombic \\
\hline Space group & Pnma \\
\hline $\mathrm{a} / \AA ̊$ & $25.227(2)$ \\
\hline $\mathrm{b} / \AA ̊ \AA$ & $29.914(3)$ \\
\hline$c / \AA ̊$ & $14.9725(12)$ \\
\hline$\alpha /^{\circ}$ & 90 \\
\hline$\beta /^{\circ}$ & 90 \\
\hline$\gamma /{ }^{\circ}$ & 90 \\
\hline Volume $/ \AA^{3}$ & $11298.7(16)$ \\
\hline Z & 4 \\
\hline$\rho_{\text {calc }} \mathrm{g} / \mathrm{cm}^{3}$ & 1.164 \\
\hline$\mu / \mathrm{mm}^{-1}$ & 4.497 \\
\hline$F(000)$ & 3952.0 \\
\hline Crystal size $/ \mathrm{mm}^{3}$ & $0.04 \times 0.035 \times 0.015$ \\
\hline Radiation & CuKa $(\lambda=1.54178)$ \\
\hline $2 \Theta$ range for data collection $/^{\circ}$ & 6.866 to 108.528 \\
\hline Index ranges & $-21 \leq h \leq 26,-31 \leq k \leq 31,-14 \leq \mathrm{I} \leq 15$ \\
\hline Reflections collected & 42902 \\
\hline Independent reflections & $7052\left[R_{\text {int }}=0.0420, R_{\text {sigma }}=0.0280\right]$ \\
\hline Data/restraints/parameters & $7052 / 5 / 475$ \\
\hline Goodness-of-fit on $F^{2}$ & 1.052 \\
\hline Final $R$ indexes $[I>=2 \sigma(I)]$ & $R_{1}=0.0588, w R_{2}=0.1687$ \\
\hline Final R indexes [all data] & $\mathrm{R}_{1}=0.0615, \mathrm{wR}_{2}=0.1711$ \\
\hline Largest diff. peak/hole / e $\AA^{-3}$ & $1.60 /-2.05$ \\
\hline
\end{tabular}


Table S16. Torsion angles between the central phenyl ring and a) the isophthalate group, $b$ and c) the adjacent benzoates in Y-thc-MOF-1.<smiles>O=C(O)c1ccc(-c2cc(-c3cc(C(=O)O)cc(C(=O)O)c3)cc(-c3ccc(C(=O)O)cc3C(=O)[O-])c2)cc1</smiles>

\begin{tabular}{|l|c|c|c|}
\hline & $\mathrm{a}\left({ }^{\circ}\right)$ & $\mathrm{b}\left({ }^{\circ}\right)^{2}$ & $\mathrm{c}\left({ }^{\circ}\right)^{3}$ \\
\hline Y-thc-MOF-1_DMF_200K & $47.5(16)$ & $48.0(17)$ & $25.9(19)$ \\
\hline Y-thc-MOF-1_EtOH_270K & $40.8(2)$ & $-54.9(17)$ & $14.7(8)$ \\
\hline Y-thc-MOF-1_EtOH_280K & $23.0(2)$ & $-45.2(8)$ & $16.1(9)$ \\
\hline Y-thc-MOF-1_EtOH_200K & $28.2(3)$ & $-42.5(2)$ & $19.4(2)$ \\
\hline Y-thc-MOF-1_EtOH_150K & $21.6(2)$ & $-48.6(3)$ & $13.3(2)$ \\
\hline Y-thc-MOF-1_EtOH_280K ${ }^{1}$ & $29.8(2)$ & $-35.3(2)$ & $6.6(2)$ \\
\hline
\end{tabular}

${ }^{1}$ Crystal was left at $100 \mathrm{~K}$, then returned to $280 \mathrm{~K} .{ }^{2}$ This benzoate group is located within the layers of the structure, denoted as $\mathbf{1}$ in the manuscript and Figure $2 \mathrm{~b} .{ }^{3}$ This benzoate groups is acting as a pillar between adjacent layers, denoted as $\mathbf{2}$ in the manuscript and Figure $2 \mathrm{~b}$.

\section{Additional structural figures}
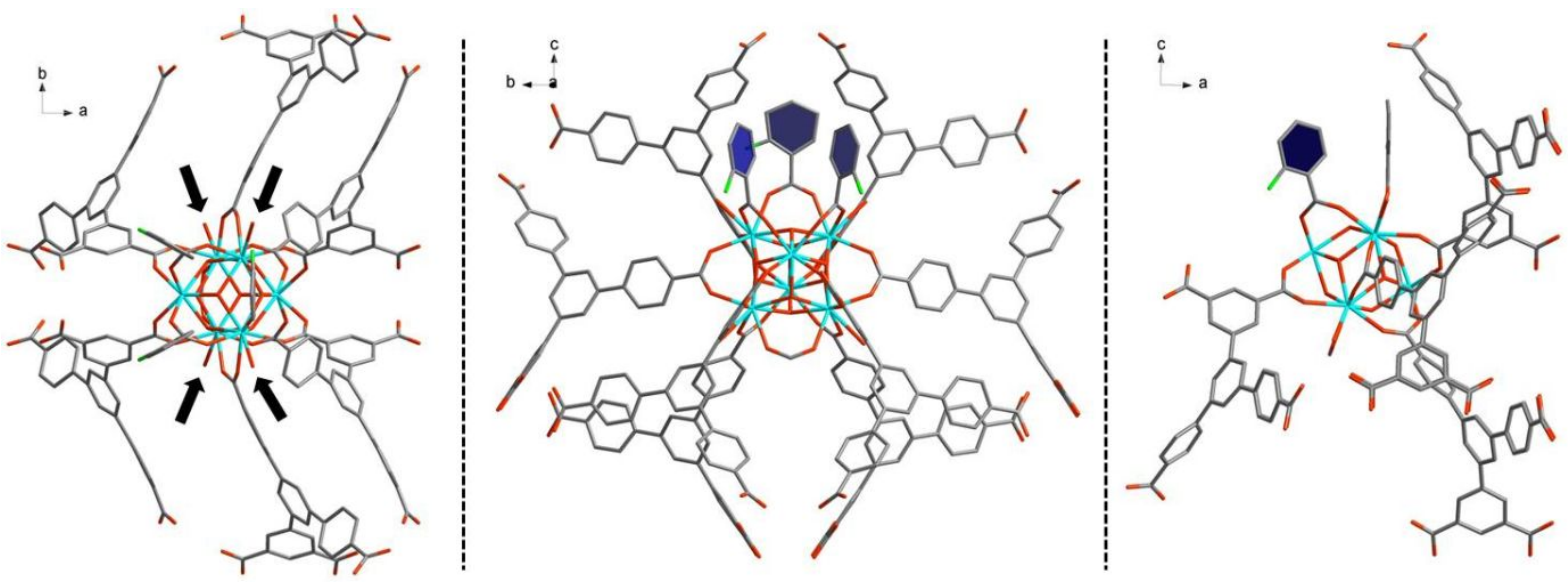

Figure S6. The coordination environment around the $\left[\mathrm{Y}_{6}\left(\mu_{3}-\mathrm{OH}\right)_{8} \mathrm{~L}_{2}\left(2-\mathrm{FC}_{6} \mathrm{H}_{4} \mathrm{COO}\right)_{3}(\mathrm{HCOO})(\mathrm{DMF})_{4}\right.$ cluster viewed at different directions. The black arrows at the left image show the position of the four coordinated DMF molecules. The coordinated 2-FBA anions are highlighted in blue. 

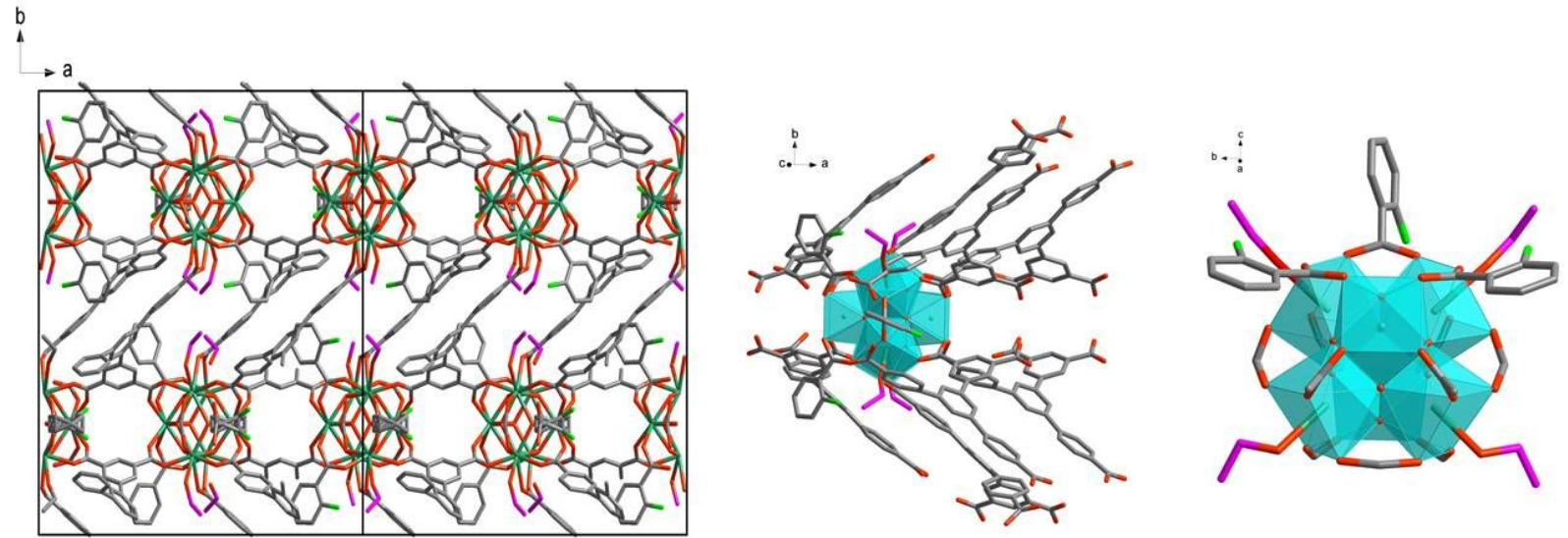

Figure S7. Single crystal structure of Tb-thc-MOF-1 exchanged with EtOH. There are four coordinated $\mathrm{EtOH}$ molecules per $\mathrm{Tb}_{6}$-cluster highlighted in pink colour. These molecules are pointing inside the interlayer spacing, along the b-axis (left and middle image). The middle image shows the coordination environment of the $\mathrm{Tb}_{6}$-cluster. In the right image, for clarity, the ligands have been removed except their coordinated carboxylate groups.

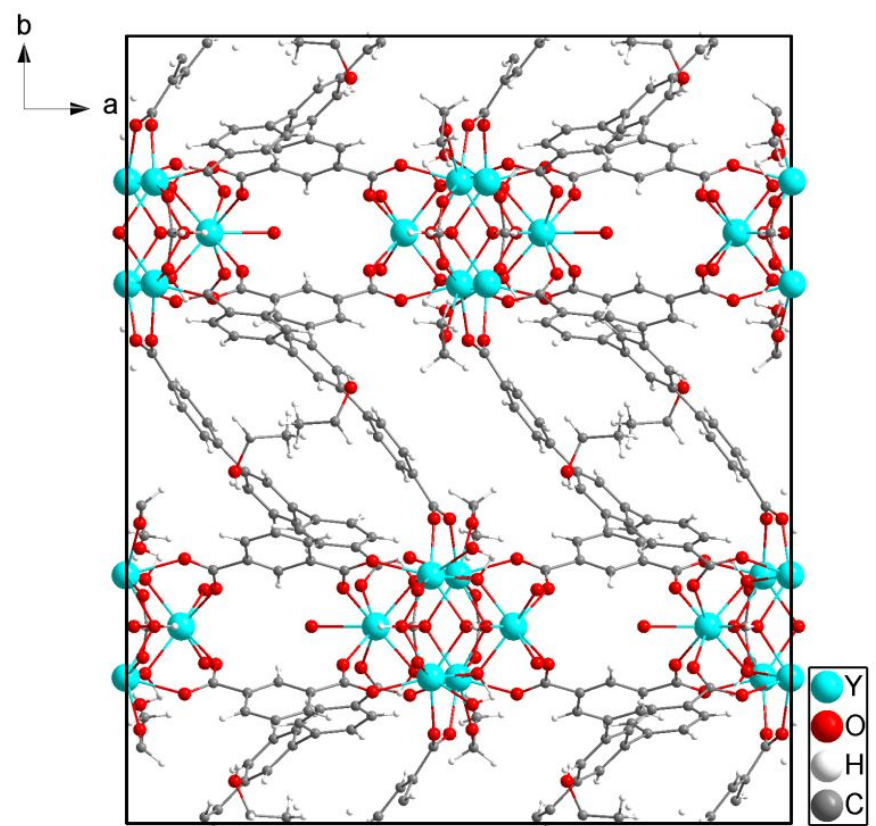

Figure S8. The structure of formate-exchanged Y-thc-MOF-1 in ethanol, showing EtOH molecules in the interlayer space. 


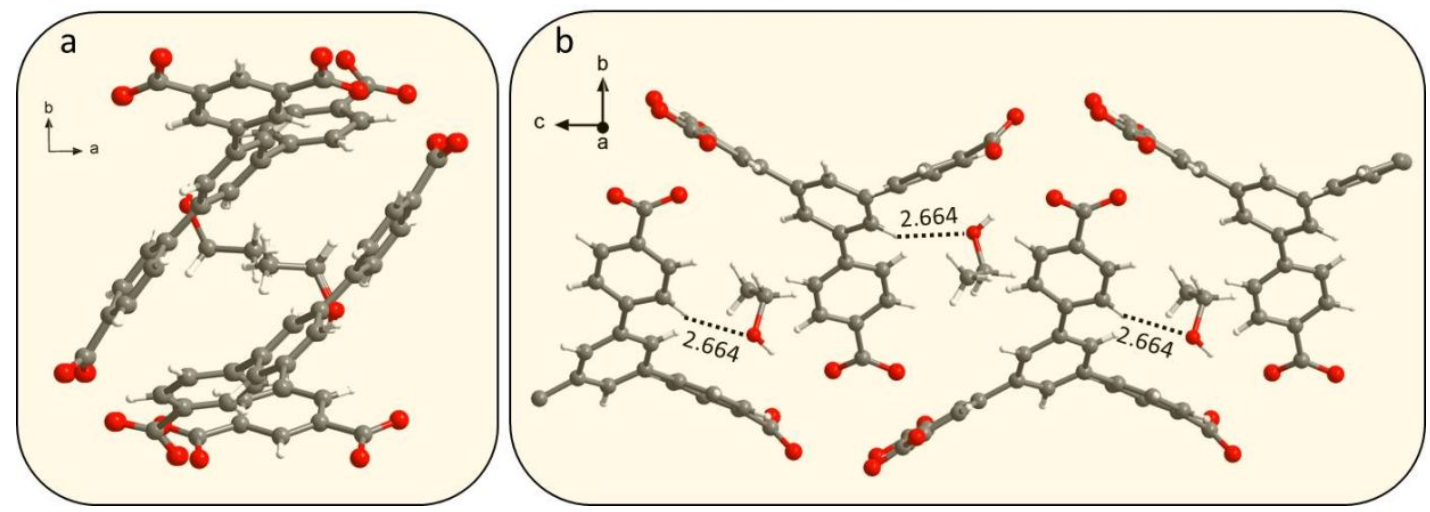

Figure S9. (a) The location of the observed EtOH molecules in formate-exchanged Y-thc-MOF-1. (b) The $\mathrm{C}-\mathrm{H} . . . \mathrm{O}$ hydrogen bond interaction between the organic linker and EtOH molecules. The $\mathrm{C}-\mathrm{H}$...O hydrogen bond distance is $2.664 \AA$ and the corresponding angle is $162.49^{\circ}$.

\section{Topological analysis}

a

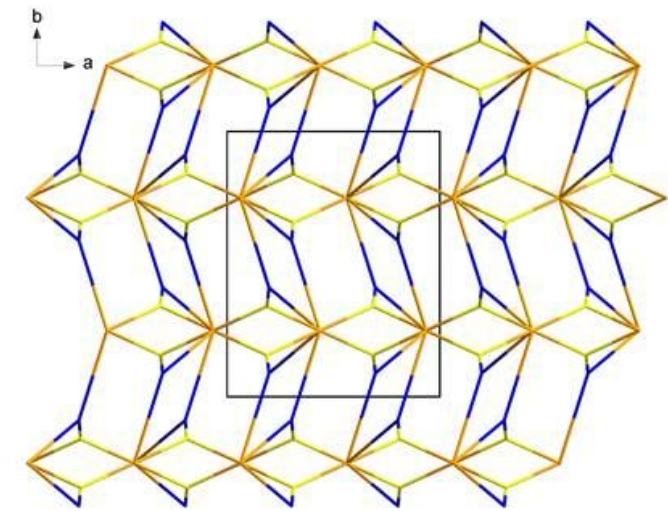

b

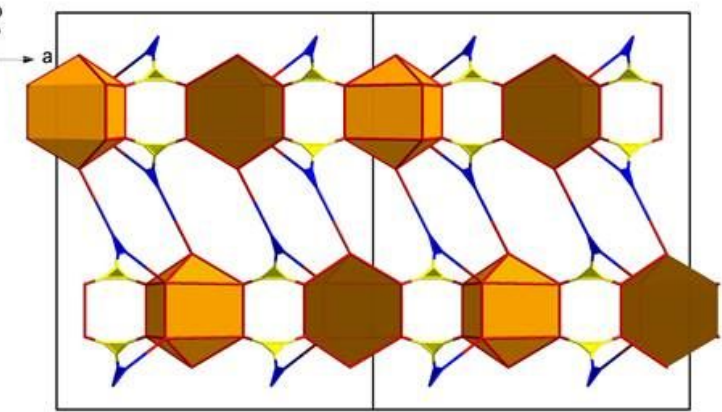

Figure S10. Topological analysis of RE-thc-MOF-1. (a) The thc net is obtained by reducing each 8-c $\mathrm{RE}_{6}$-cluster to a 8-c node (orange), while each tetratopic ligand is represented by two interconnected non-symmetrical 3-c triangular nodes (yellow and blue). (b) The augmented thc-a net.

The topological analysis results are summarized below:

TD10=1558

Vertex symbols for selected sublattice

3-c node (yellow) Point symbol:\{4.5.6\}

Extended point symbol:[4.5.6(2)]

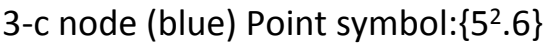

Extended point symbol:[5.5.6(2)]

8-c node (orange) Point symbol: $\left\{4^{2} \cdot 5^{4} \cdot 6^{3} \cdot 7^{6} \cdot 8^{13}\right\}$

Extended point symbol:

[4.4.5.5.5.5.6.6.6(2).7.7.7(2).7(2).7(3).7(3).8.8.8.8(2).8(2).8(2).8(2).8(4).8(4).8(4).8(4).8(6).8(6)]

Point symbol for net: $\{4.5 .6\} 2\left\{4^{2} \cdot 5^{4} \cdot 6^{3} \cdot 7^{6} \cdot 8^{13}\right\}\left\{5^{2} \cdot 6\right\} 2$

$3,3,8-c$ net with stoichiometry (3-c)2(3-c)2(8-c); 3-nodal net 


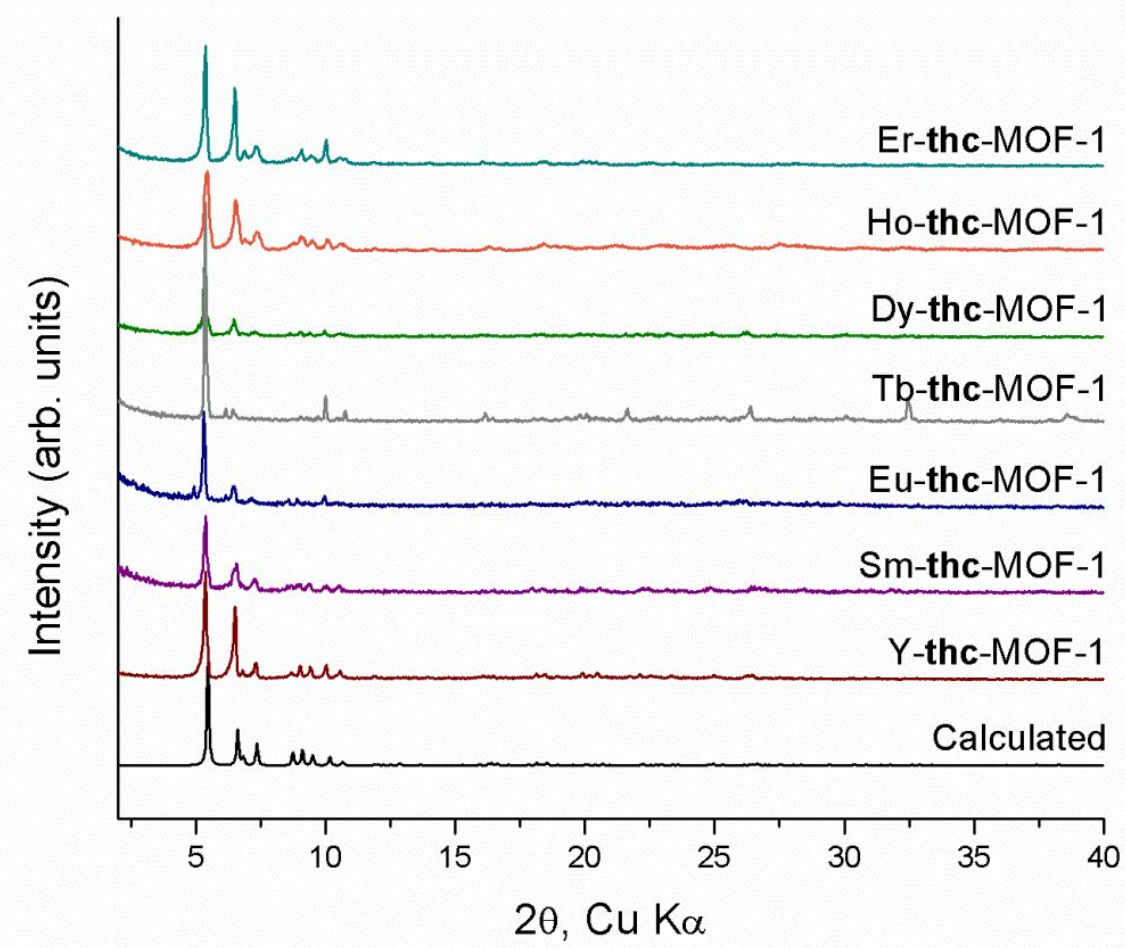

Figure S11. Experimental PXRD patterns of as-made RE-thc-MOF-1 solids along with the calculated pattern obtained from the single crystal structure of Y-thc-MOF-1.

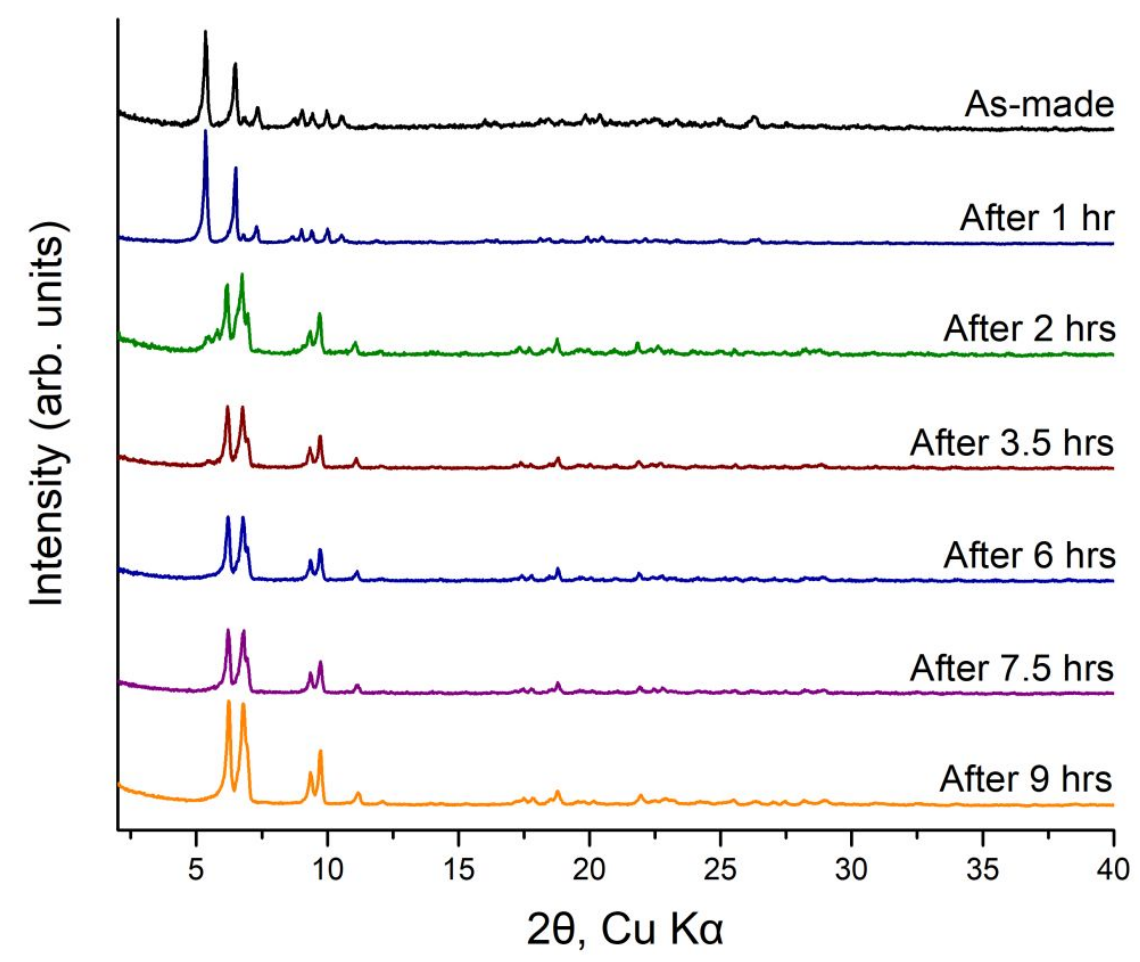

Figure S12. Experimental PXRD patterns of as-made Y-thc-MOF-1 over time. 


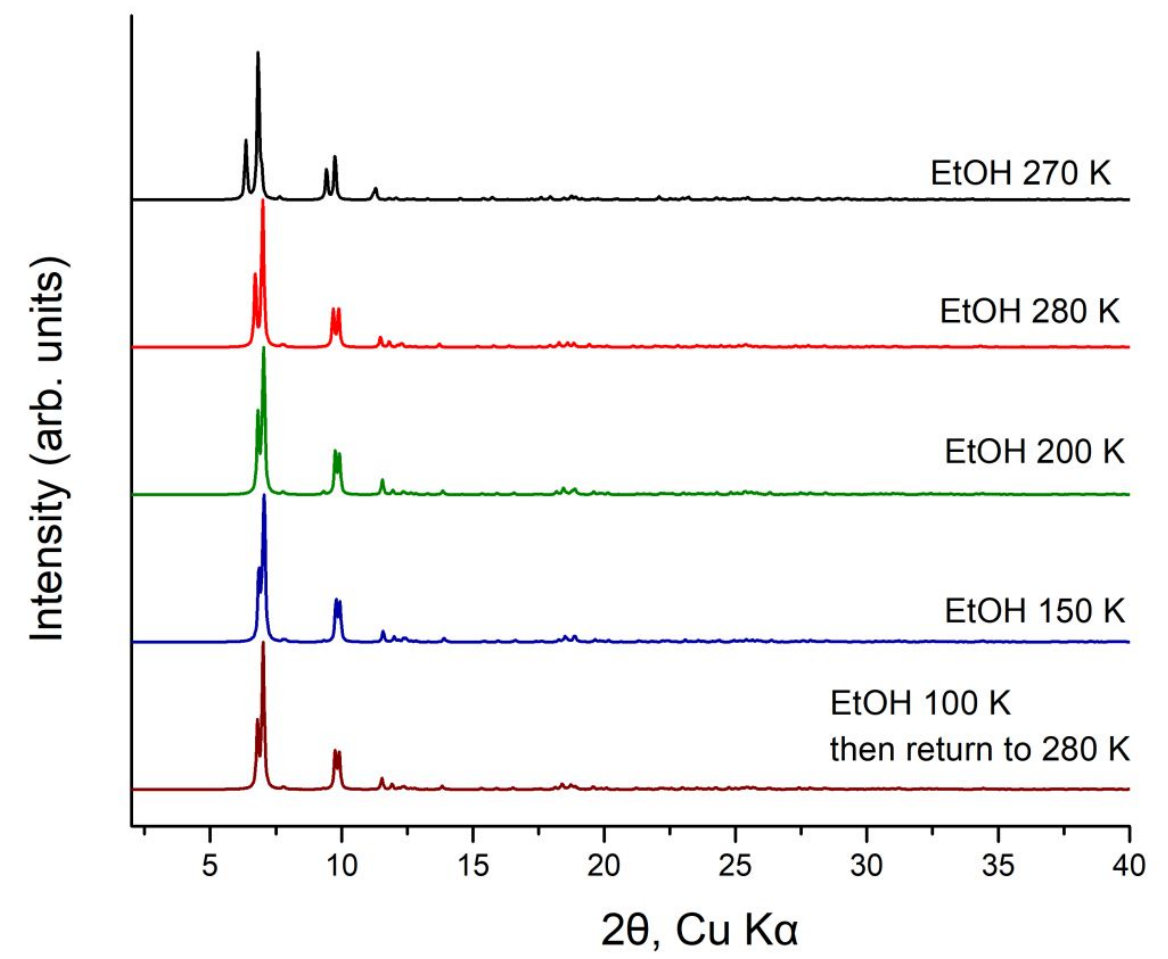

Figure S13. Calculated PXRD patterns obtained from the single crystal structures of ethanolexchanged Y-thc-MOF-1 at the indicated temperatures.

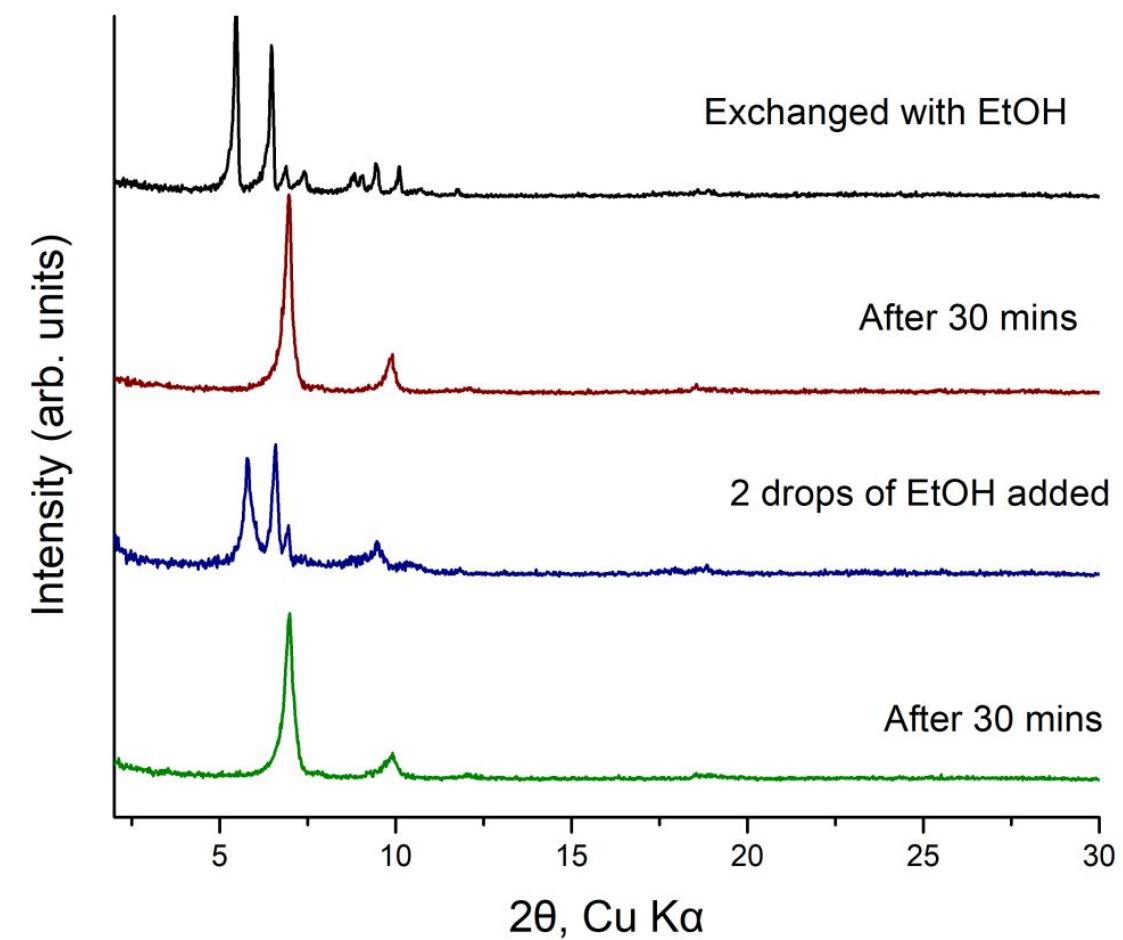

Figure S14. Experimental PXRD patterns obtained from the ethanol-exchanged Y-thc-MOF-1 sample (black), after its exposure in air for $30 \mathrm{~min}$ (red), after the addition of ethanol to the air exposed sample (blue) and its pattern after exposure in air for 30 mins (green). 


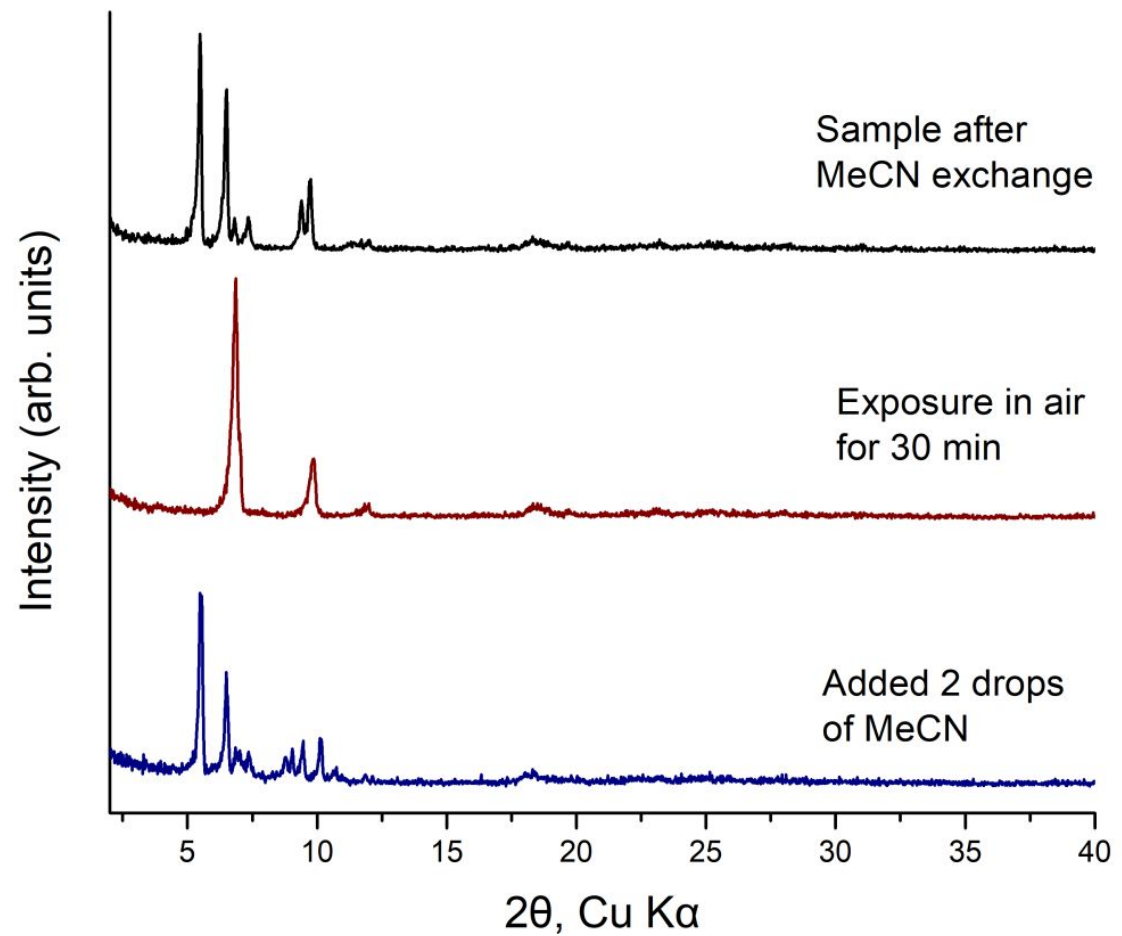

Figure S15. Experimental PXRD patterns of MeCN-exchanged Y-thc-MOF-1 (black), after its exposure in air for $30 \mathrm{~min}$ (red), after the addition of 2 drops of $\mathrm{MeCN}$ to the air-dried sample (blue).

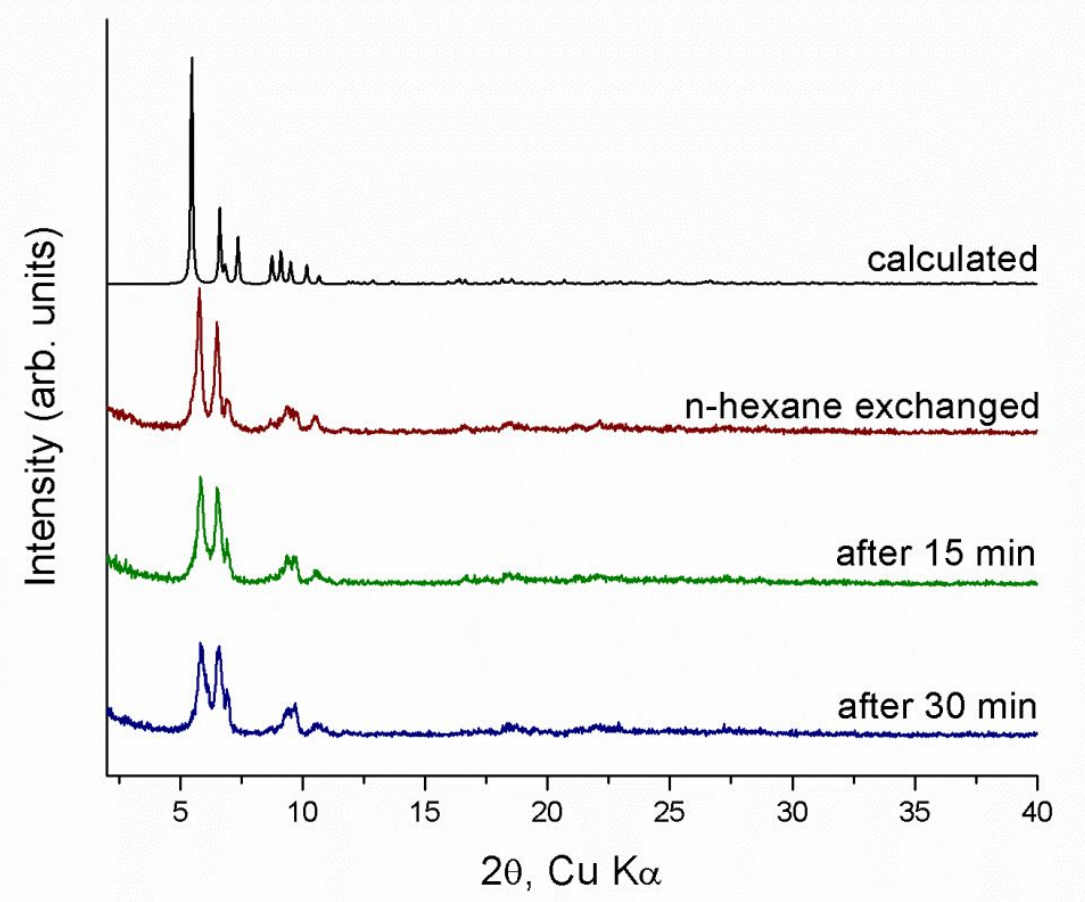

Figure S16. Experimental PXRD patterns of $n$-hexane exchanged $Y$-thc-MOF-1 over time, along with the calculated pattern of as-made Y-thc-MOF-1. 


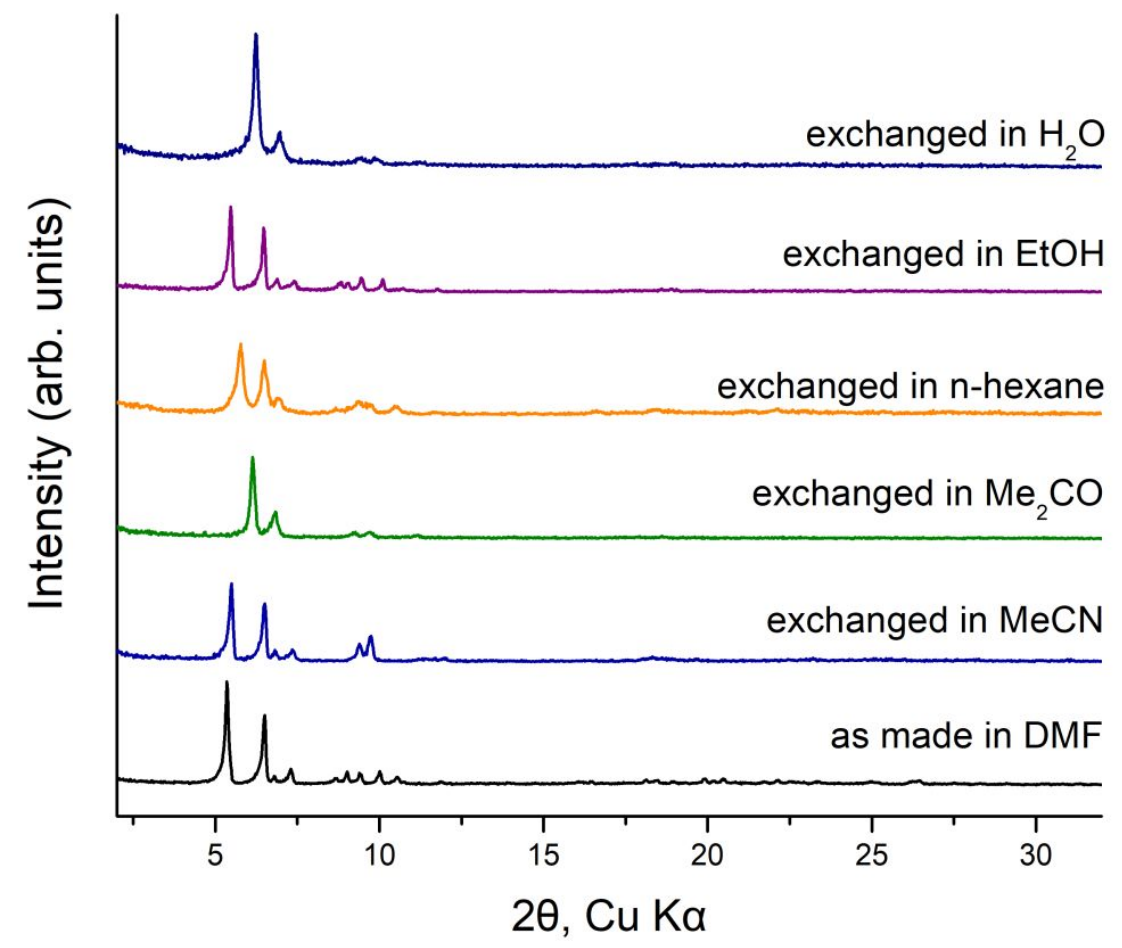

Figure S17. Experimental PXRD pattern of as-made Y-thc-MOF-1 (black), along with the PXRD pattern after exchanging the material with acetonitrile (blue), acetone (green), n-hexane (orange), ethanol (purple) and water (dark blue).

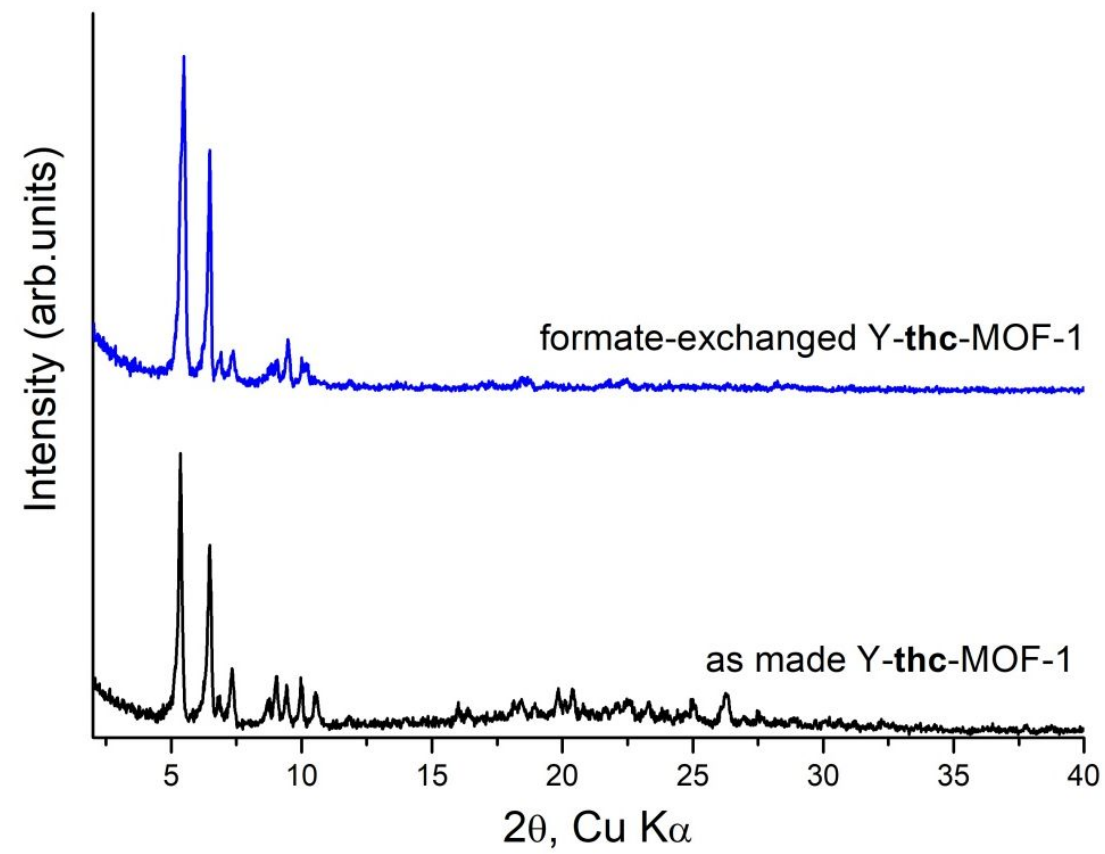

Figure S18. Experimental PXRD pattern of formate-exchanged Y-thc-MOF-1 (blue), along with the corresponding pattern of as-made Y-thc-MOF-1(black). 


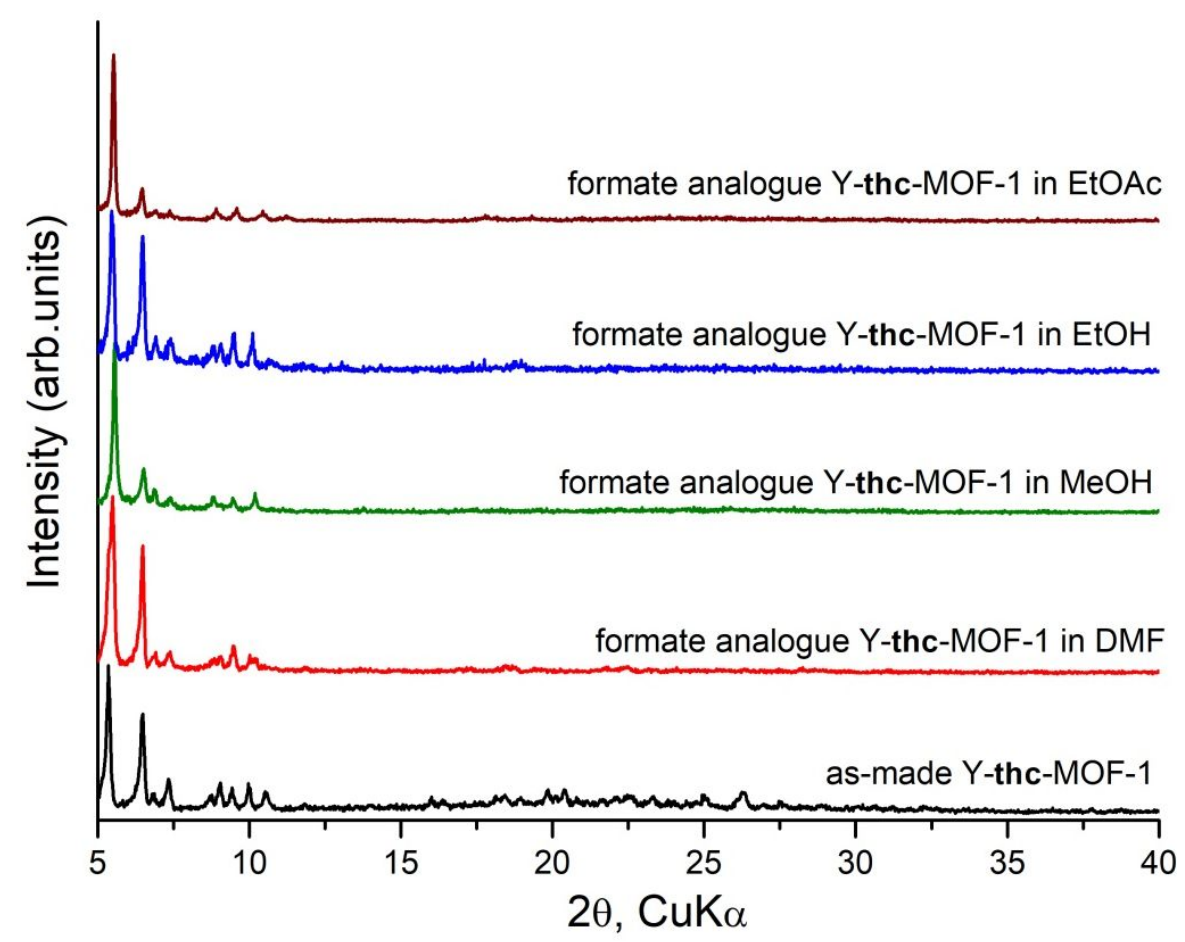

Figure S19. Experimental PXRD pattern of the formate analogue of Y-thc-MOF-1 at different polar solvents.

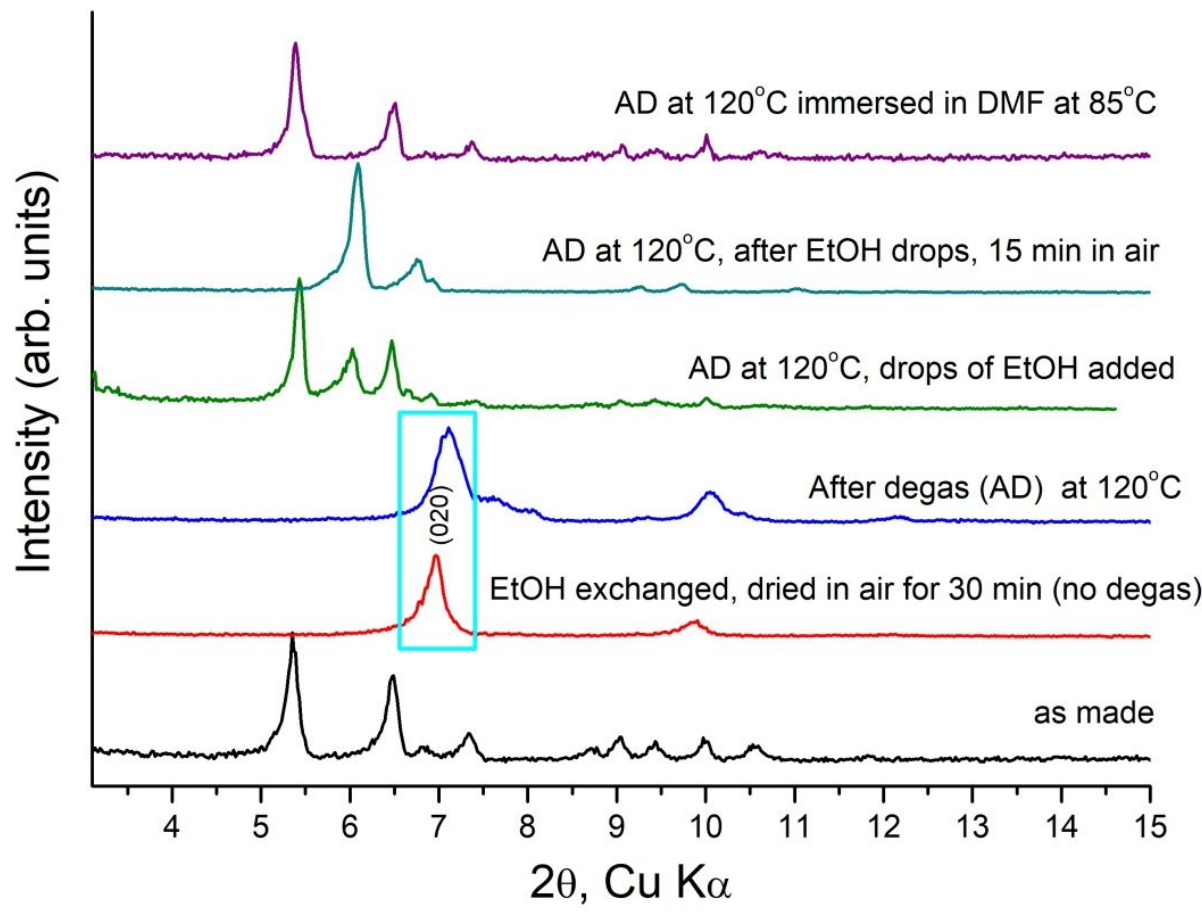

Figure S20. Experimental PXRD pattern of the Y-thc-MOF-1 after degas (AD) at $120^{\circ} \mathrm{C}$ (blue line) along with the corresponding pattern of EtOH-exchanged sample dried in open air for $30 \mathrm{~min}$ (red line). The rectangular box highlights the fact that the (020) reflection in the activated sample at 120 ${ }^{\circ} \mathrm{C}$ appears at slightly higher $2 \theta$ angle, indicating a more contracted phase. This phase displays a fully reversibly breathing behavior upon addition of EtOH and DMF solvents, as shown in the corresponding PXRD patterns. 


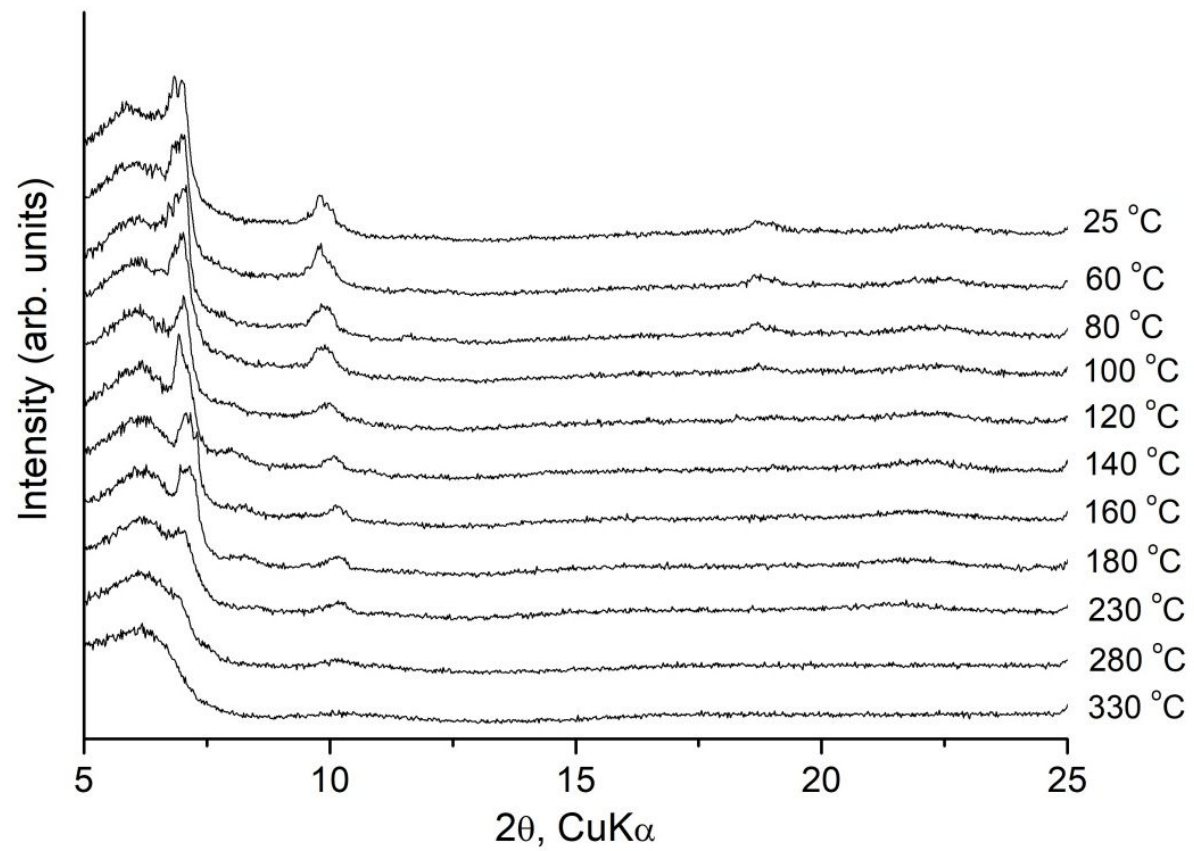

Figure S21. Variable temperature PXRD of EtOH-exchanged Y-thc-MOF-1 recorder under vacuum. The heating rate was $1^{\circ} \mathrm{C} / \mathrm{min}$. The broad low angle peak is an instrumental artifact. 
Gas and vapor sorption measurements

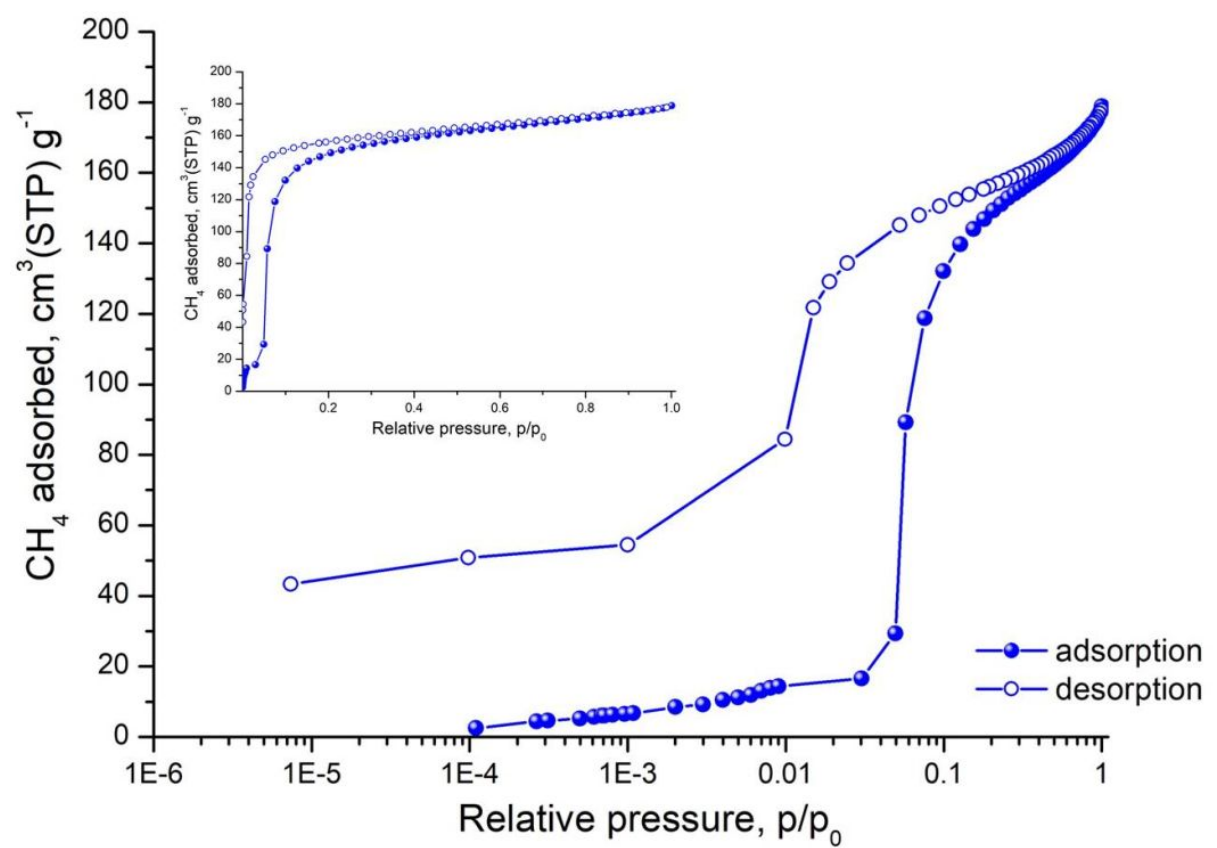

Figure S22. $\mathrm{CH}_{4}$ sorption isotherm in semi-logarithmic scale of Y-thc-MOF-1 recorded at $112 \mathrm{~K}$. Inset shows the isotherm in linear scale. The desorption curve remains open down to $7.4 \times 10^{-6}$ relative pressure. The total pore volume at $0.99 \mathrm{p} / \mathrm{p}_{0}$ is $0.30 \mathrm{~cm}^{3} \mathrm{~g}^{-1}$. The percent of the trapped $\mathrm{CH}_{4}$ is $24 \%$.

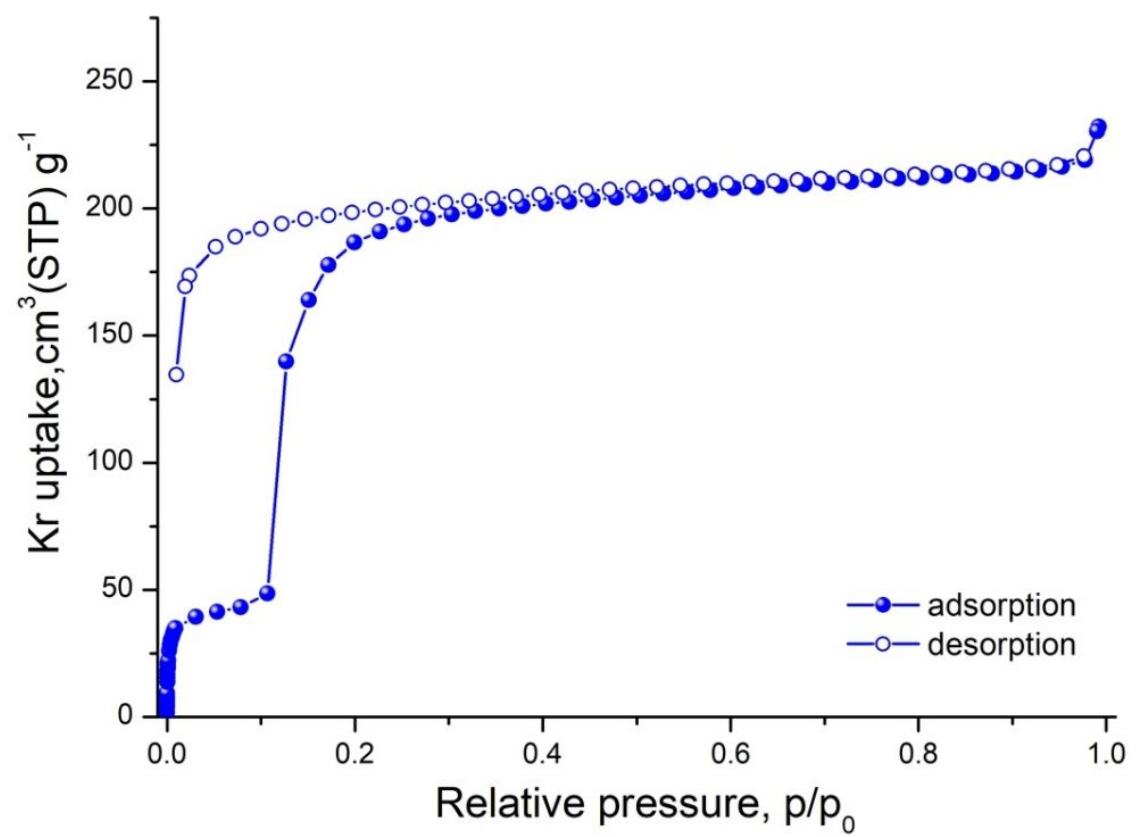

Figure S23. $\mathrm{Kr}$ sorption isotherm of Y-thc-MOF-1 recorded at $120 \mathrm{~K}$. The total pore volume at 0.99 $\mathrm{p} / \mathrm{p}_{0}$ is $0.33 \mathrm{~cm}^{3} \mathrm{~g}^{-1}$. 


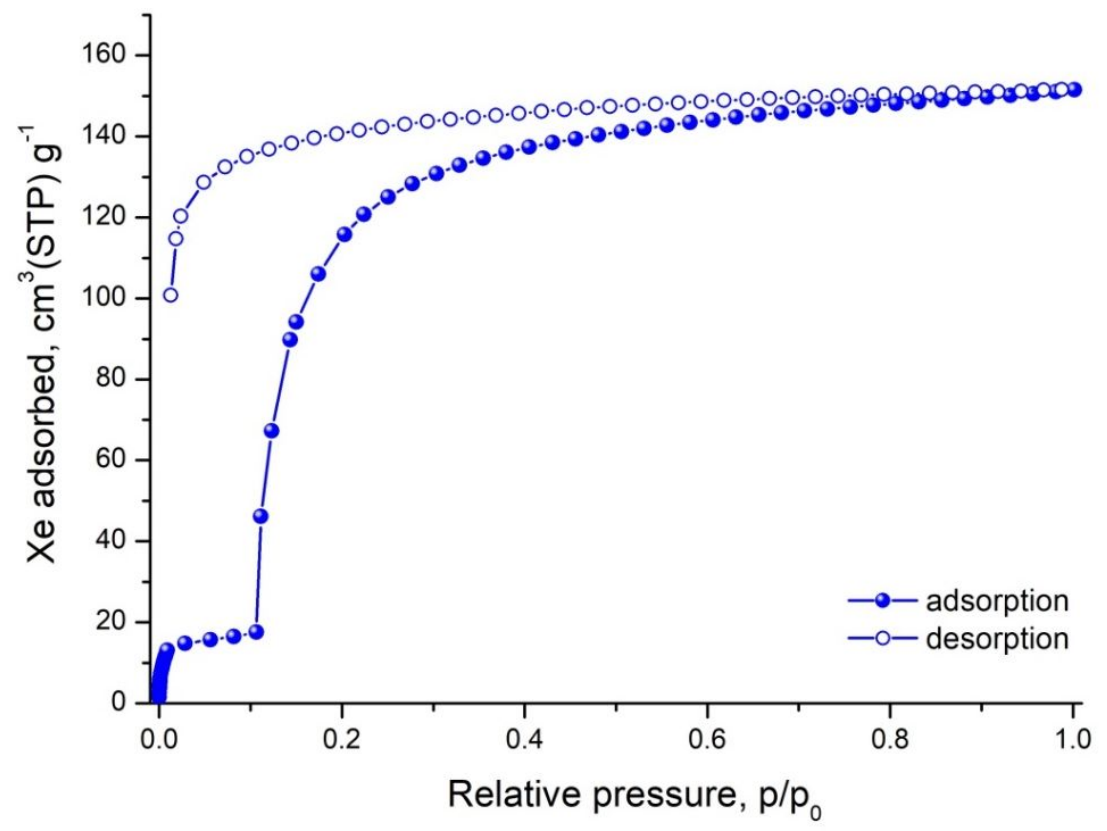

Figure S24. Xe sorption isotherm of Y-thc-MOF-1 recorded at $166 \mathrm{~K}$. The total pore volume at 0.99 $\mathrm{p} / \mathrm{p}_{0}$ is $0.30 \mathrm{~cm}^{3} \mathrm{~g}^{-1}$.

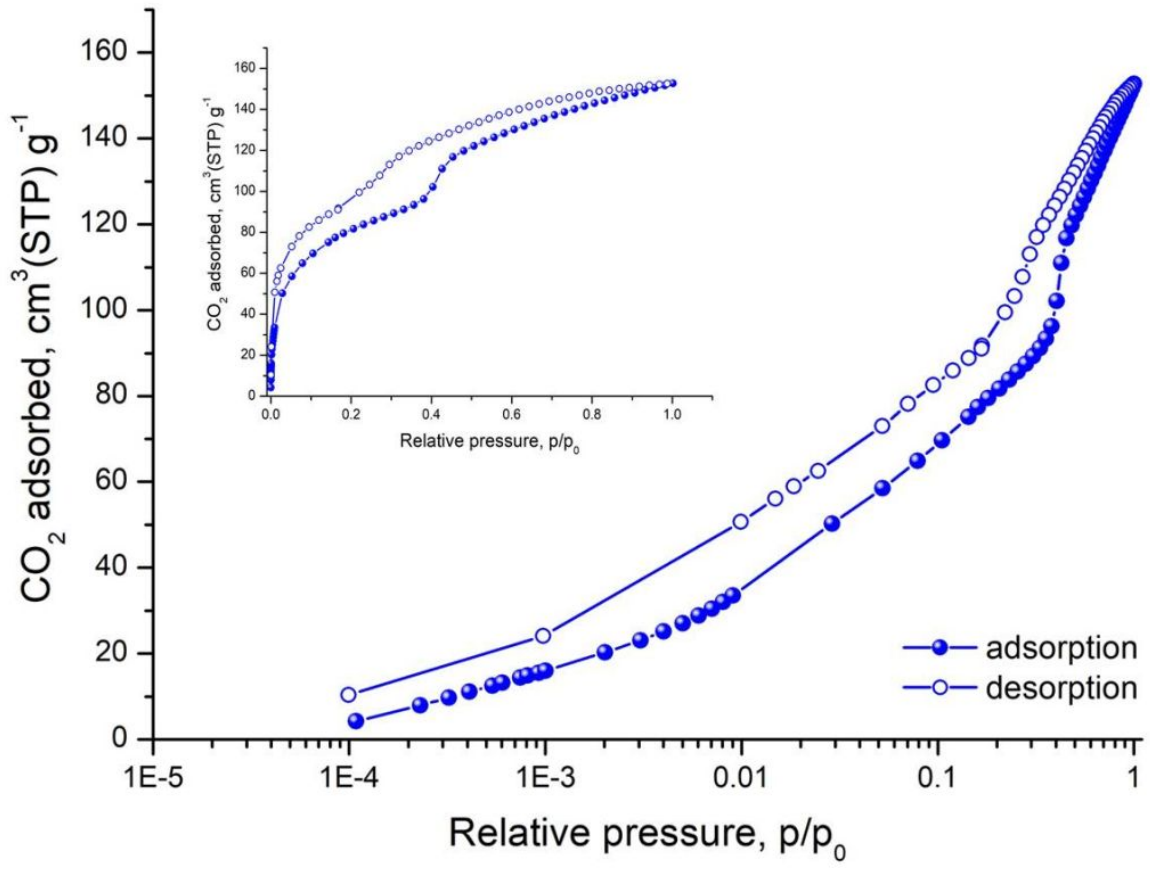

Figure S25. $\mathrm{CO}_{2}$ sorption isotherm of $\mathrm{Y}$-thc-MOF-1 recorded at $195 \mathrm{~K}$. The total pore volume at 0.99 $\mathrm{p} / \mathrm{p}_{0}$ is $0.24 \mathrm{~cm}^{3} \mathrm{~g}^{-1}$. 


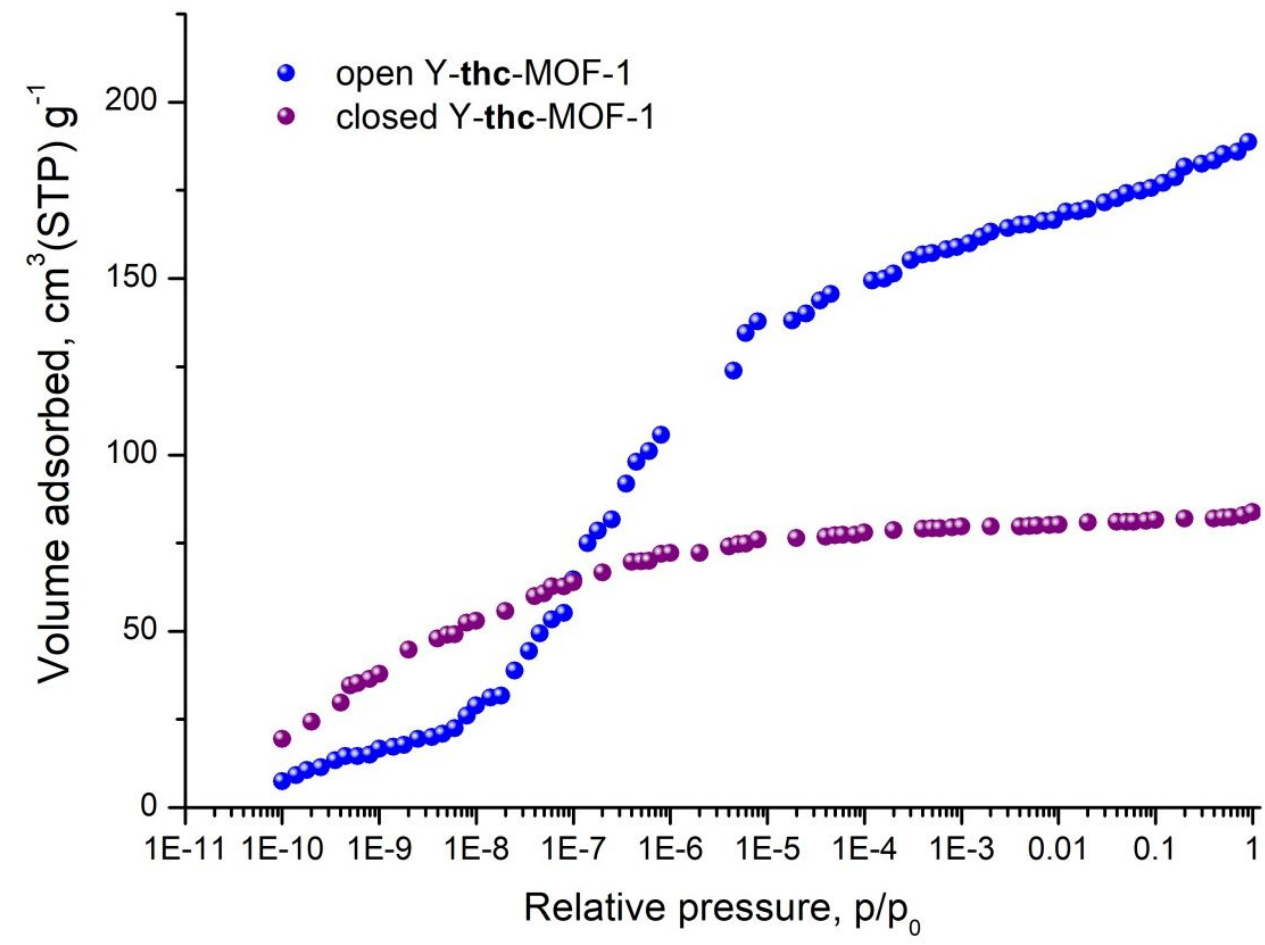

Figure S26. Nitrogen adsorption isotherms at $77 \mathrm{~K}$ of open (blue) and closed (purple) Y-thc-MOF-1 obtained by GCMC calculations.

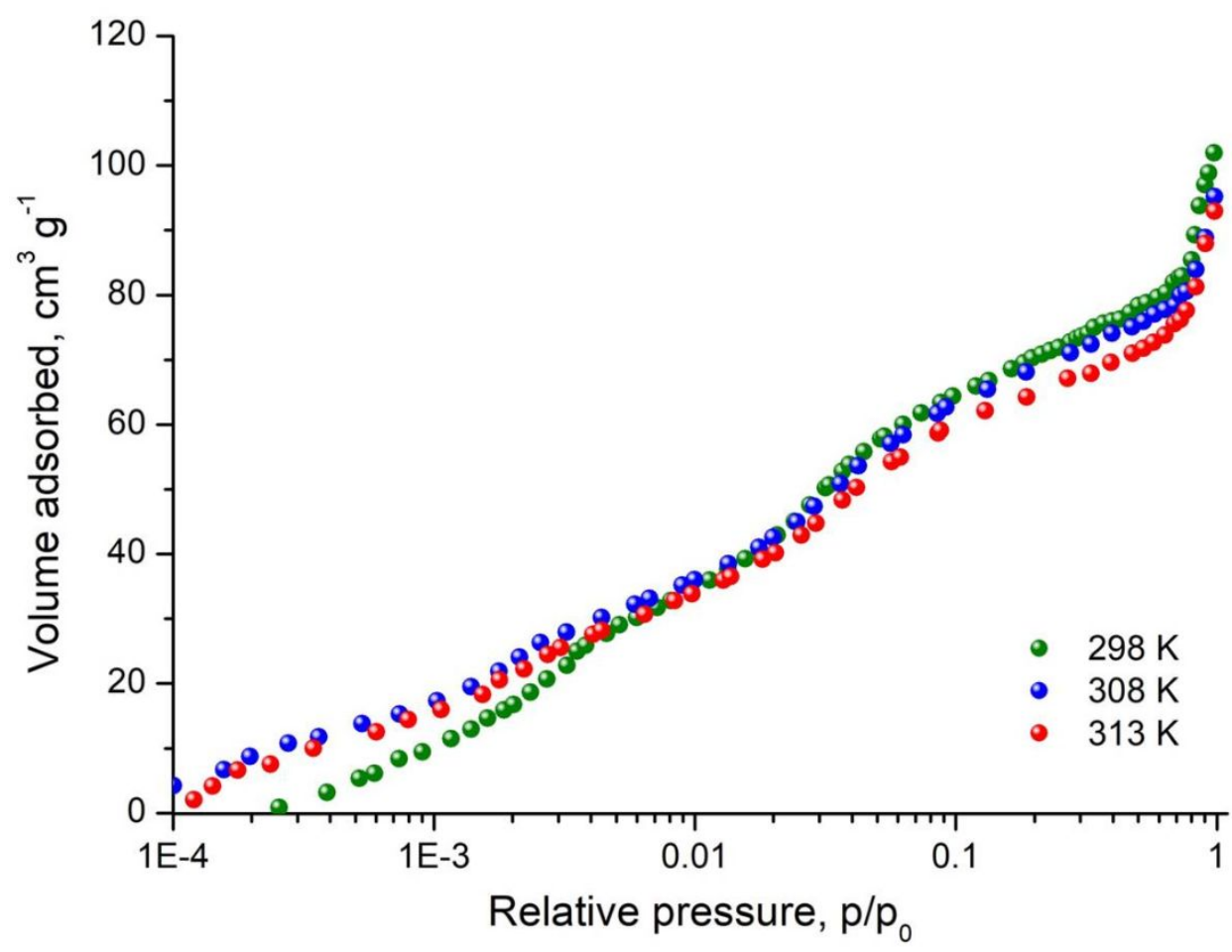

Figure S27. Ethanol adsorption isotherms of Y-thc-MOF-1 at the indicated temperatures. 


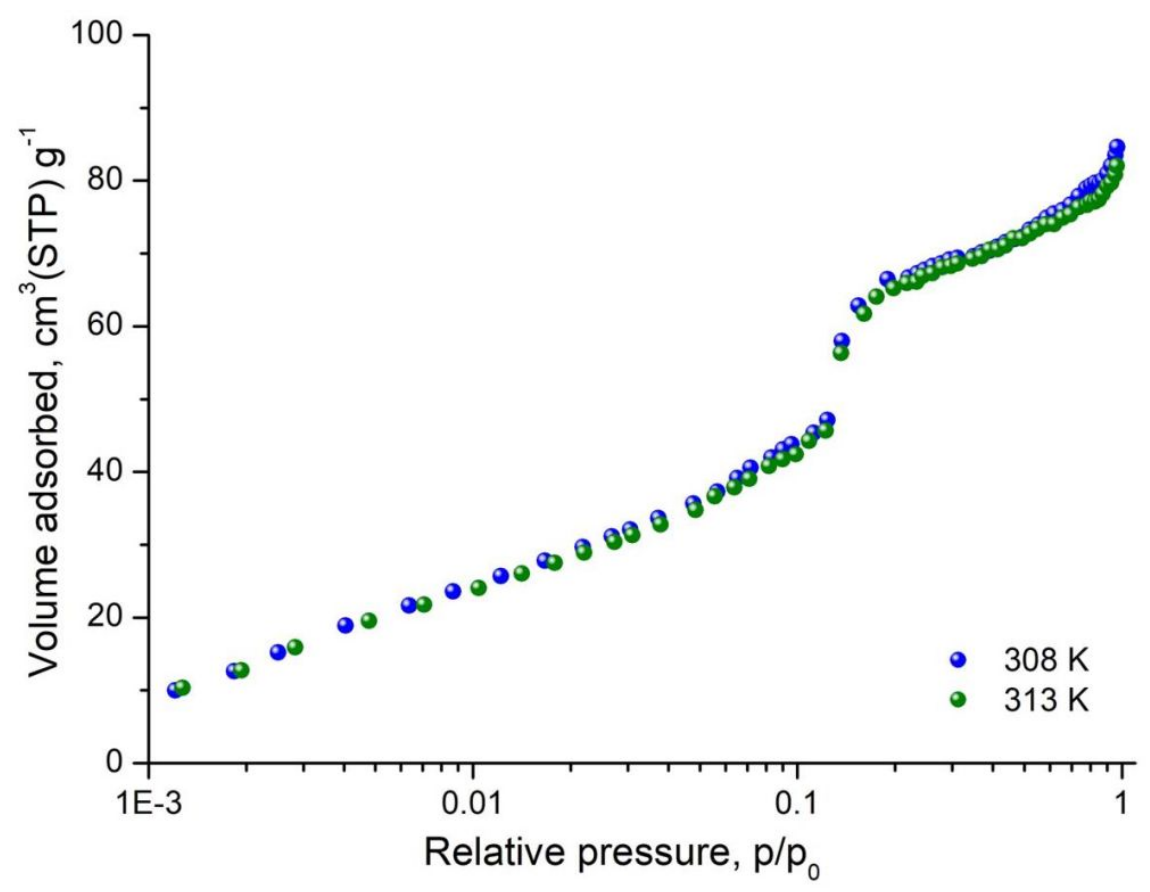

Figure S28. Acetonitrile adsorption isotherms of Y-thc-MOF-1 at the indicated temperatures.

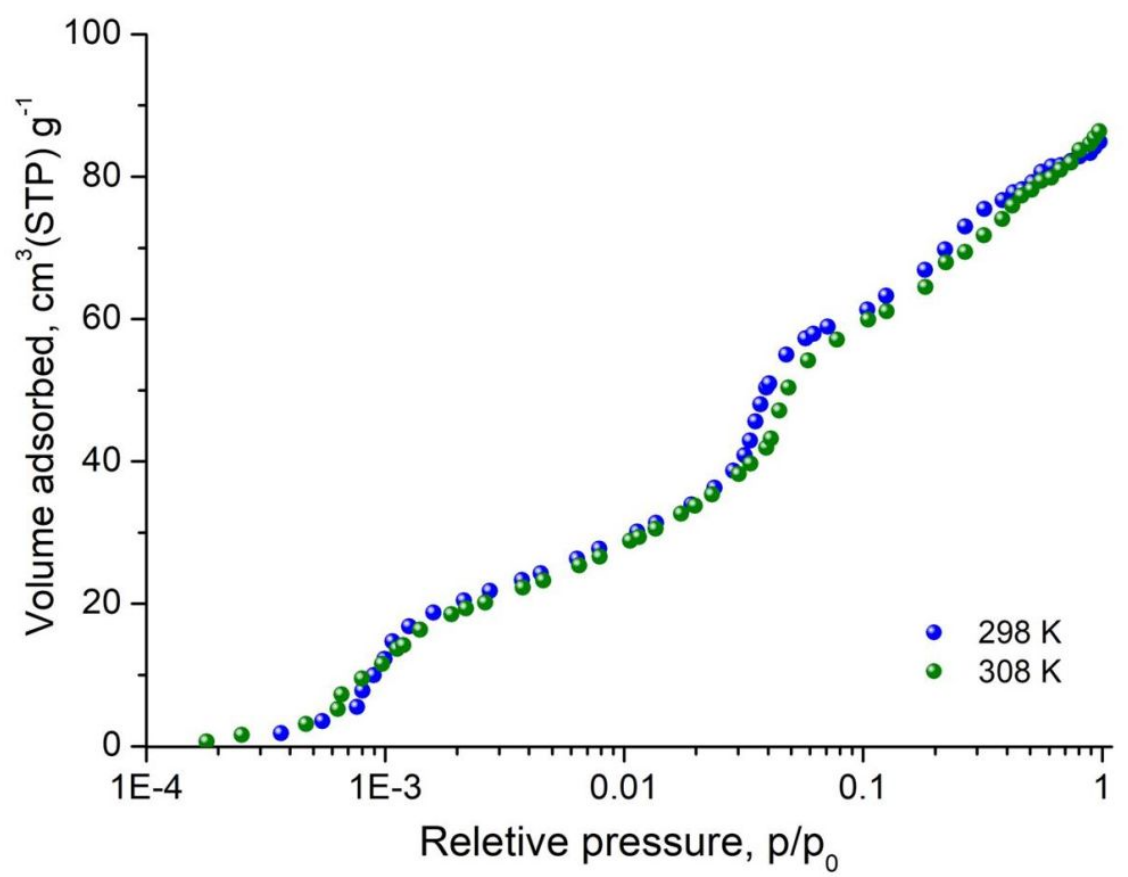

Figure S29. Benzene adsorption isotherms of Y-thc-MOF-1 at the indicated temperatures. 


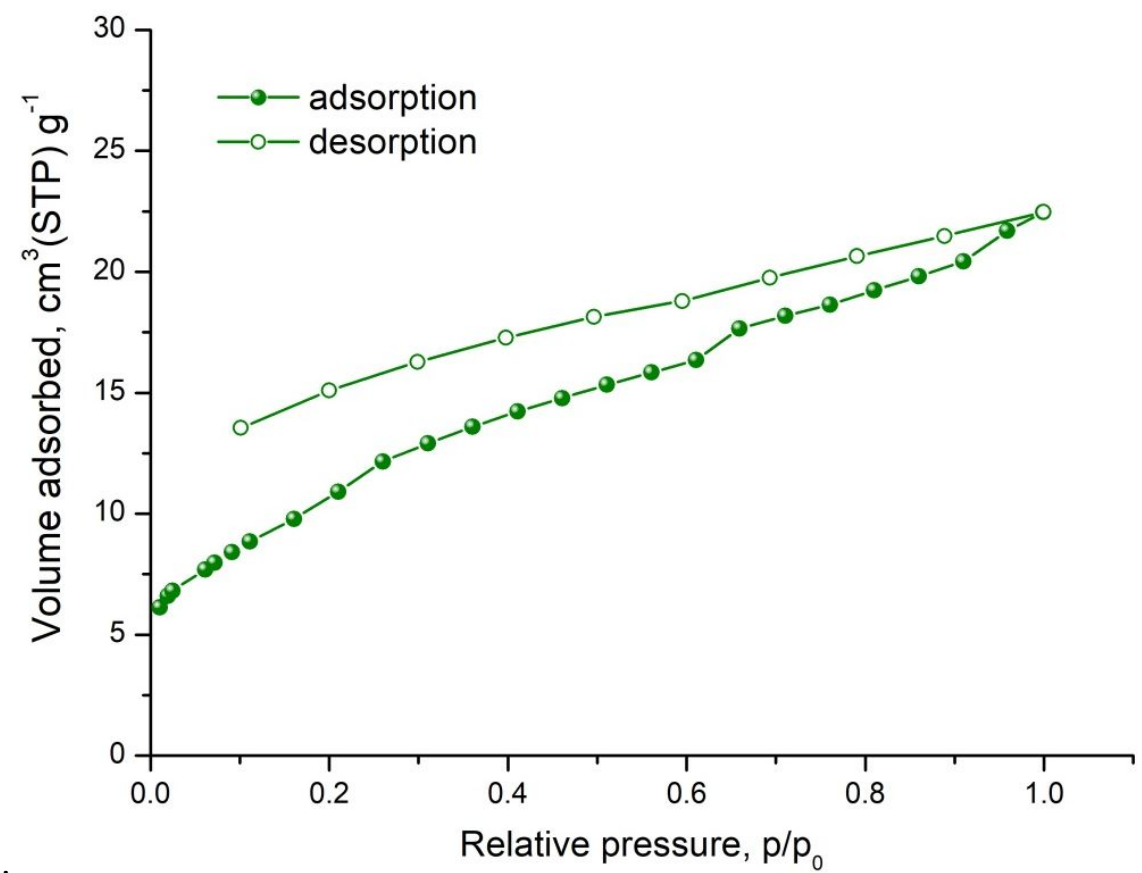

Figure S30. Nitrogen sorption isotherm of formate-exchanged Y-thc-MOF-1, recorded at $77 \mathrm{~K}$. The sample was activated at $40^{\circ} \mathrm{C}$.

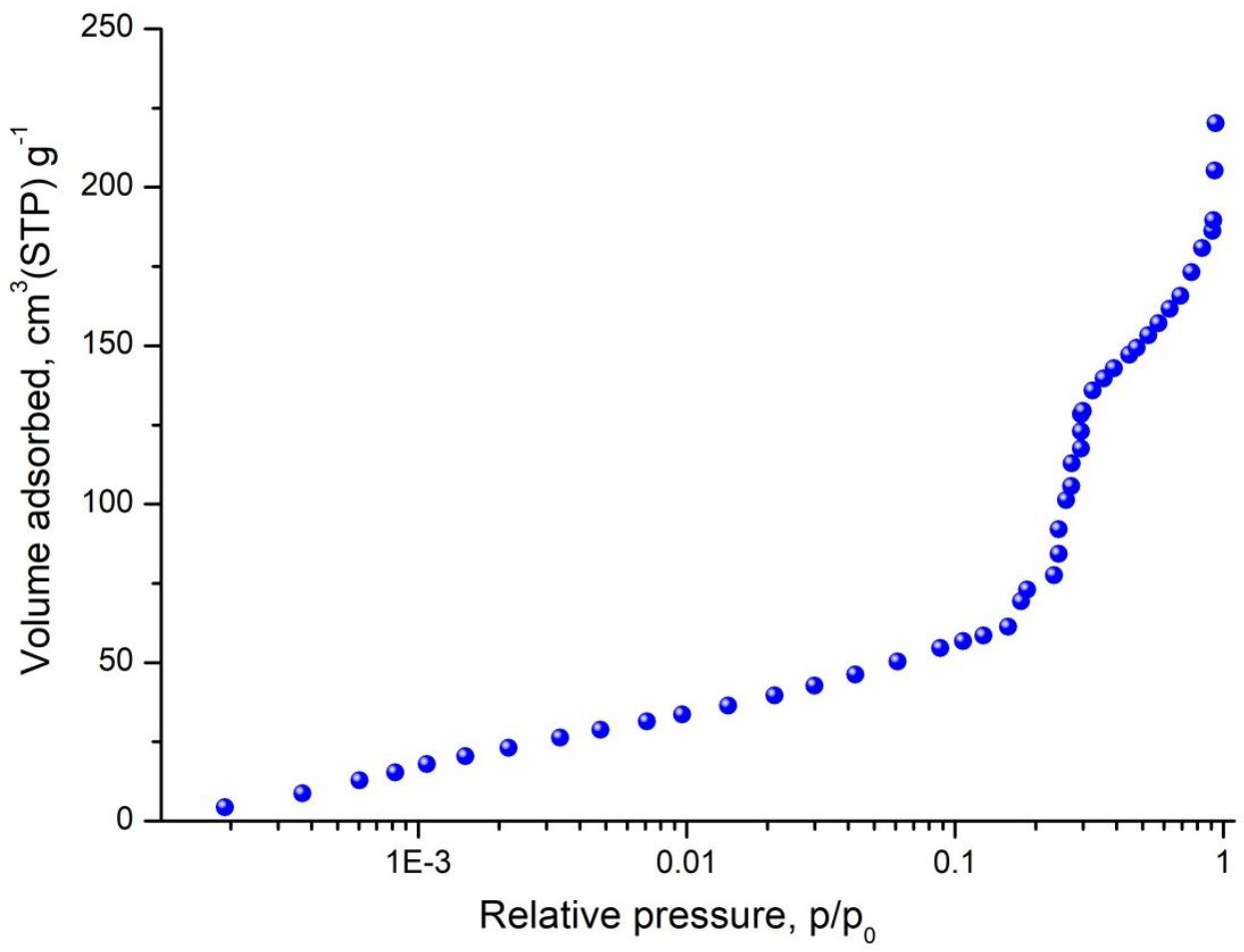

Figure S31. Methanol adsorption isotherm of formate-exchanged Y-thc-MOF-1, recorded at $298 \mathrm{~K}$. The total pore volume at saturation is $0.39 \mathrm{~cm}^{3} \mathrm{~g}^{-1}$ which is consistent with the corresponding value obtained from the EtOH adsorption isotherm $\left(0.37 \mathrm{~cm}^{3} \mathrm{~g}^{-1}\right)$, recorded at the same conditions (Figure 5 in the manuscript). 


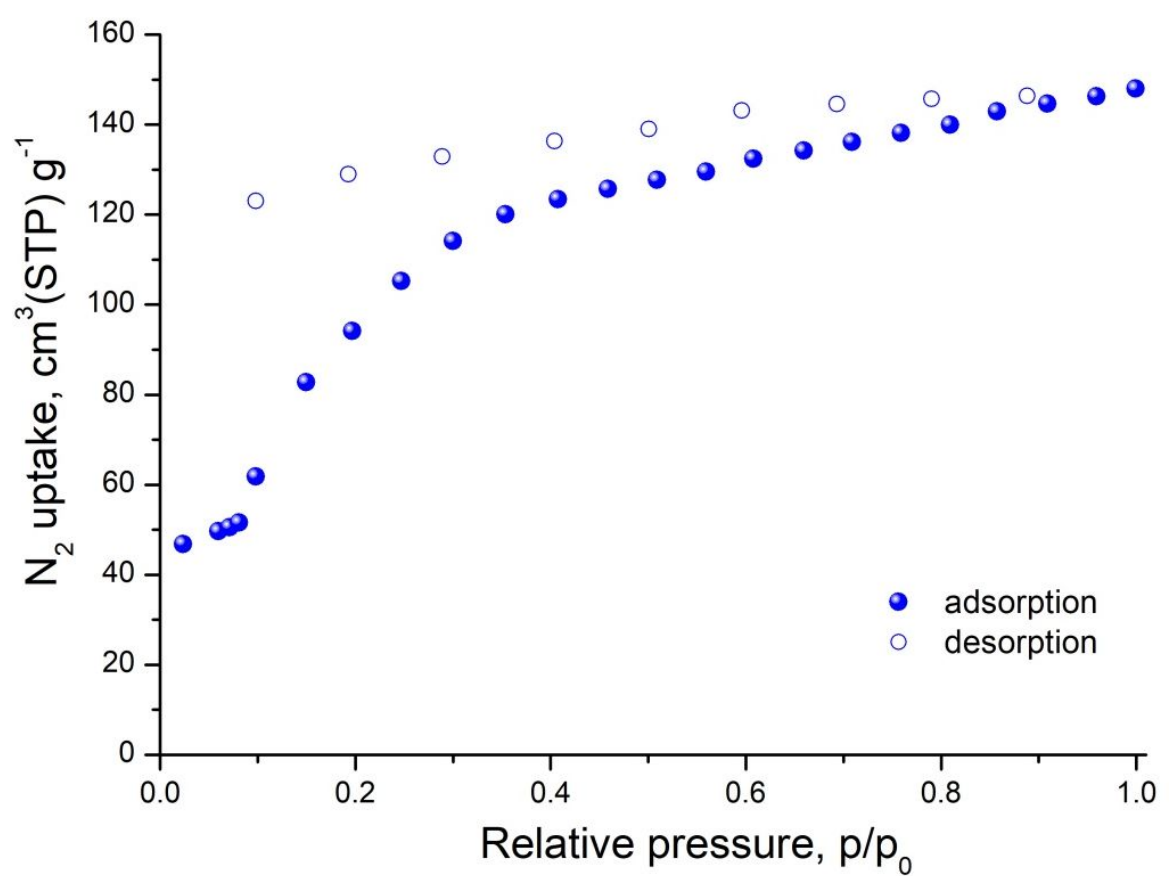

Figure S32. Nitrogen sorption isotherm of Dy-thc-MOF-1 recorded at $77 \mathrm{~K}$.

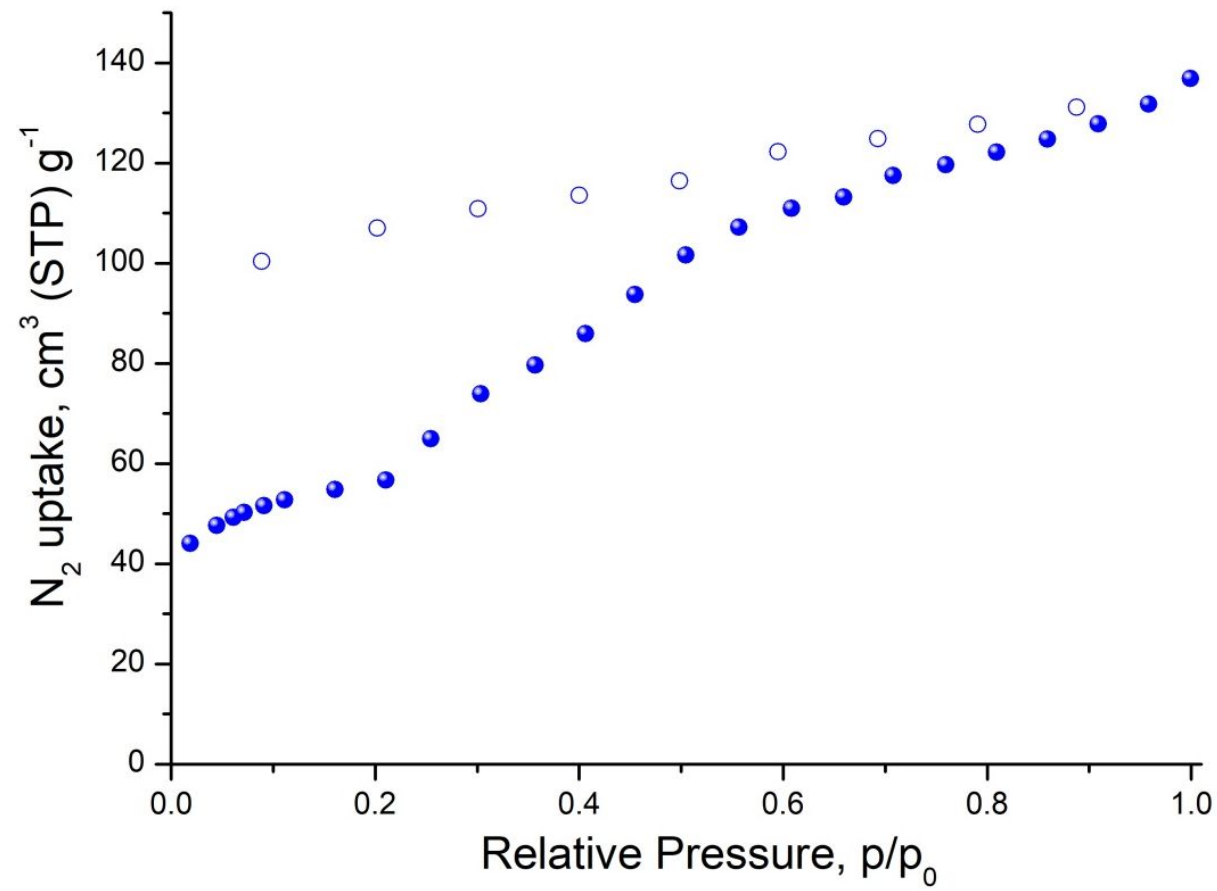

Figure S33. Nitrogen sorption isotherm of Tb-thc-MOF-1 recorded at $77 \mathrm{~K}$. 


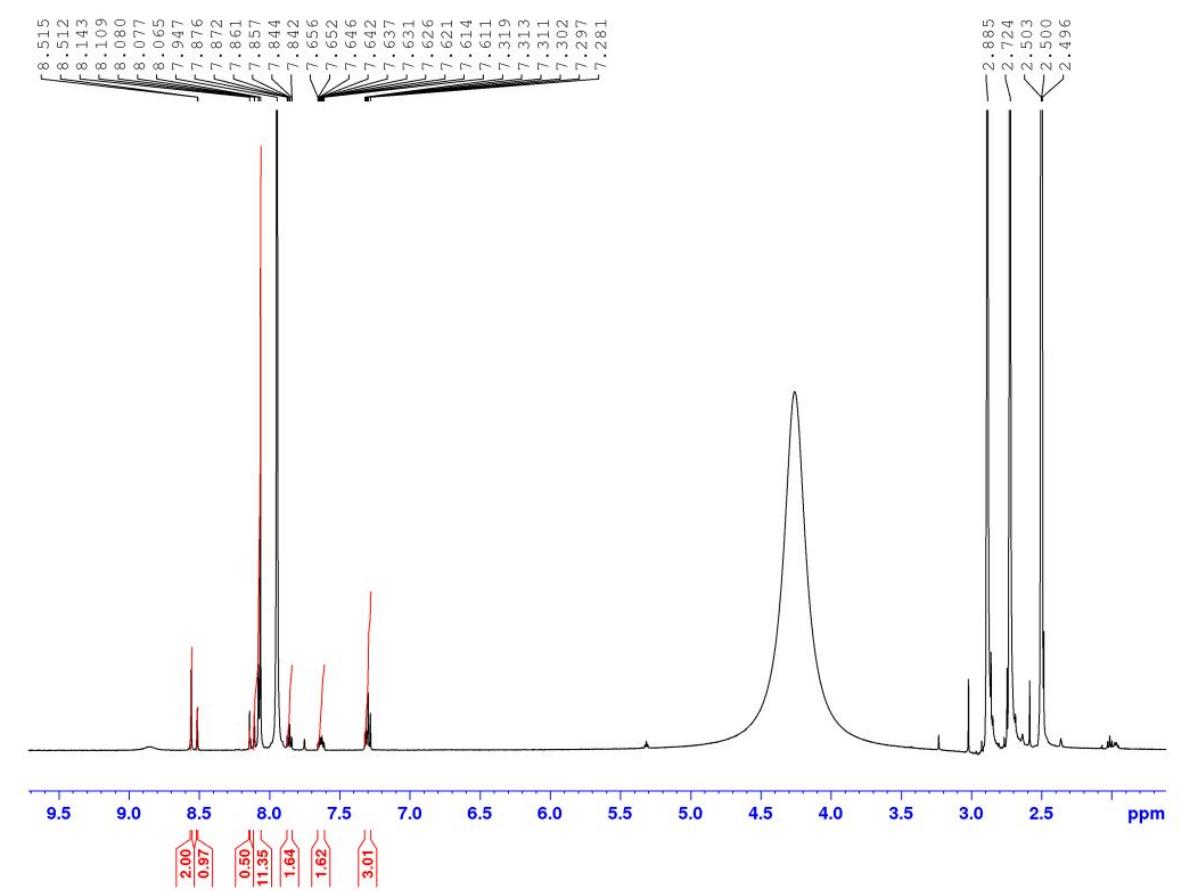

Figure S34. ${ }^{1} \mathrm{H}$ NMR spectrum of as-made Y-thc-MOF-1 digested in $\mathrm{HCl} / \mathrm{DMSO}-\mathrm{d}_{6}$ solution. The integration of peaks confirms the presence of $\mathbf{L}^{4-}, 2$-fluorobenzoate and formate molecules in a respective 2:3:1 ratio as observed by SCXRD.

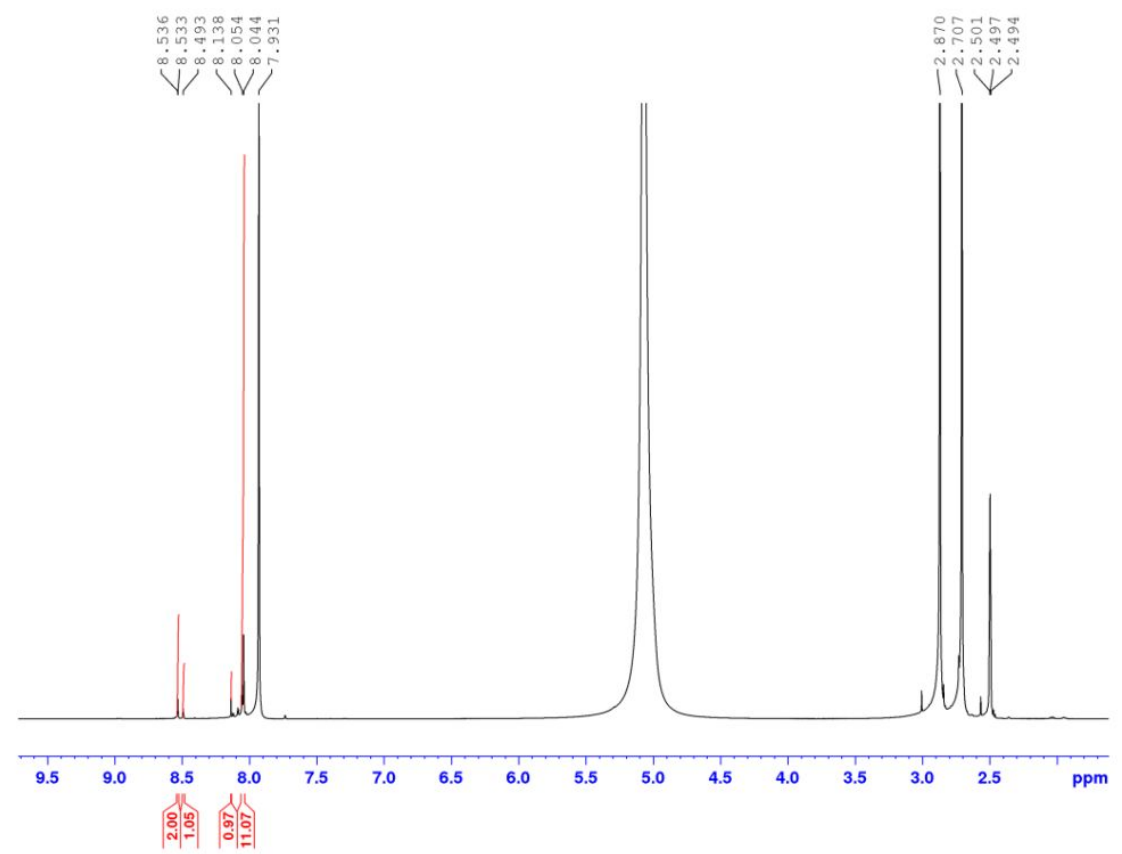

Figure S35. ${ }^{1} \mathrm{H}$ NMR spectrum of Y-thc-MOF-1, after post-synthetic exchange of 2-fluorobenzoates with formates (digested in $\mathrm{HCl} / \mathrm{DMSO}_{-} \mathrm{d}_{6}$ solution). The absence of any peak corresponding to 2fluorobenzoates indicates that full exchange with formates has occurred. 


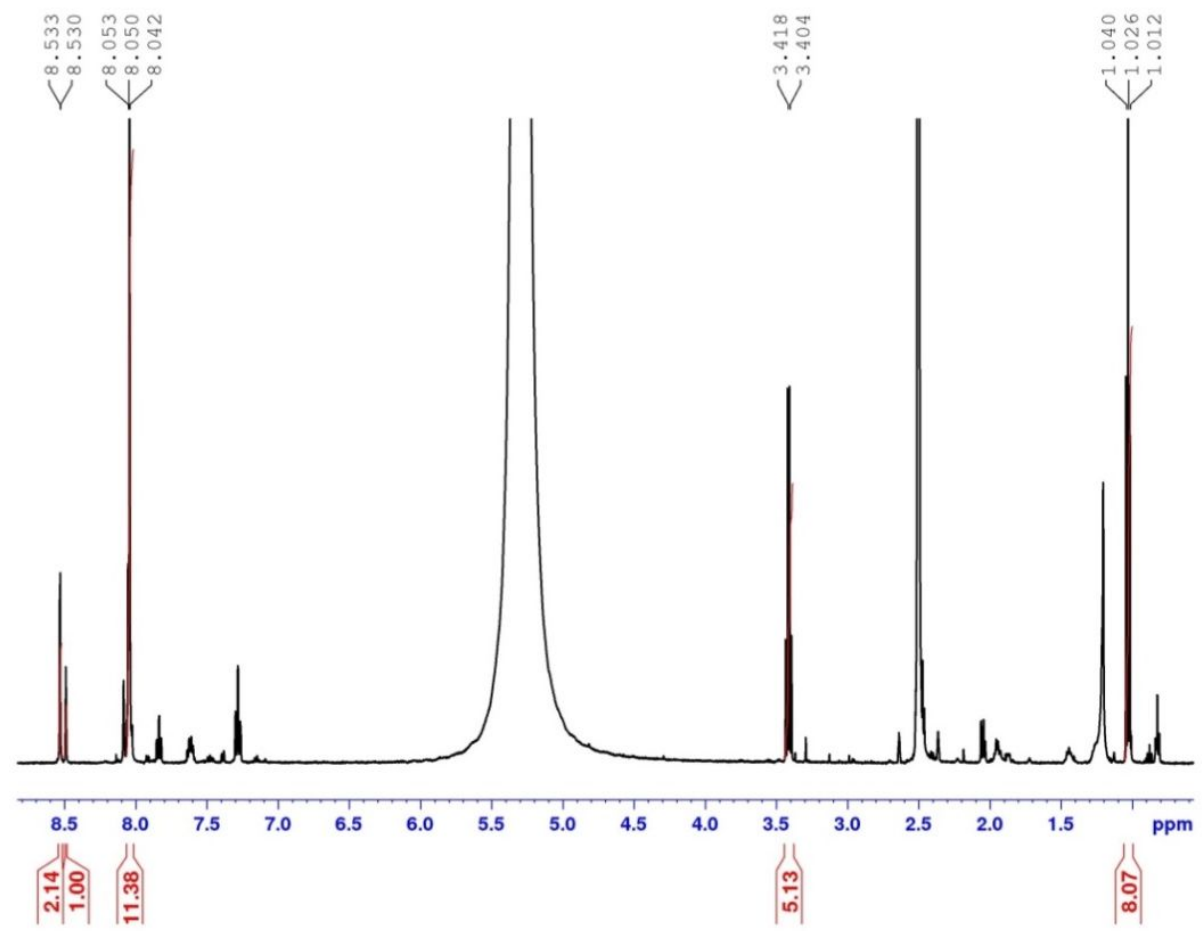

Figure S36. ${ }^{1} \mathrm{H}$ NMR spectrum of EtOH-exchanged Y-thc-MOF-1 (digested in $\mathrm{HCl} / \mathrm{DMSO}-\mathrm{d}_{6}$ solution), after activation under dynamic vacuum at room temperature. The ratio of EtOH per cluster is 5:1.

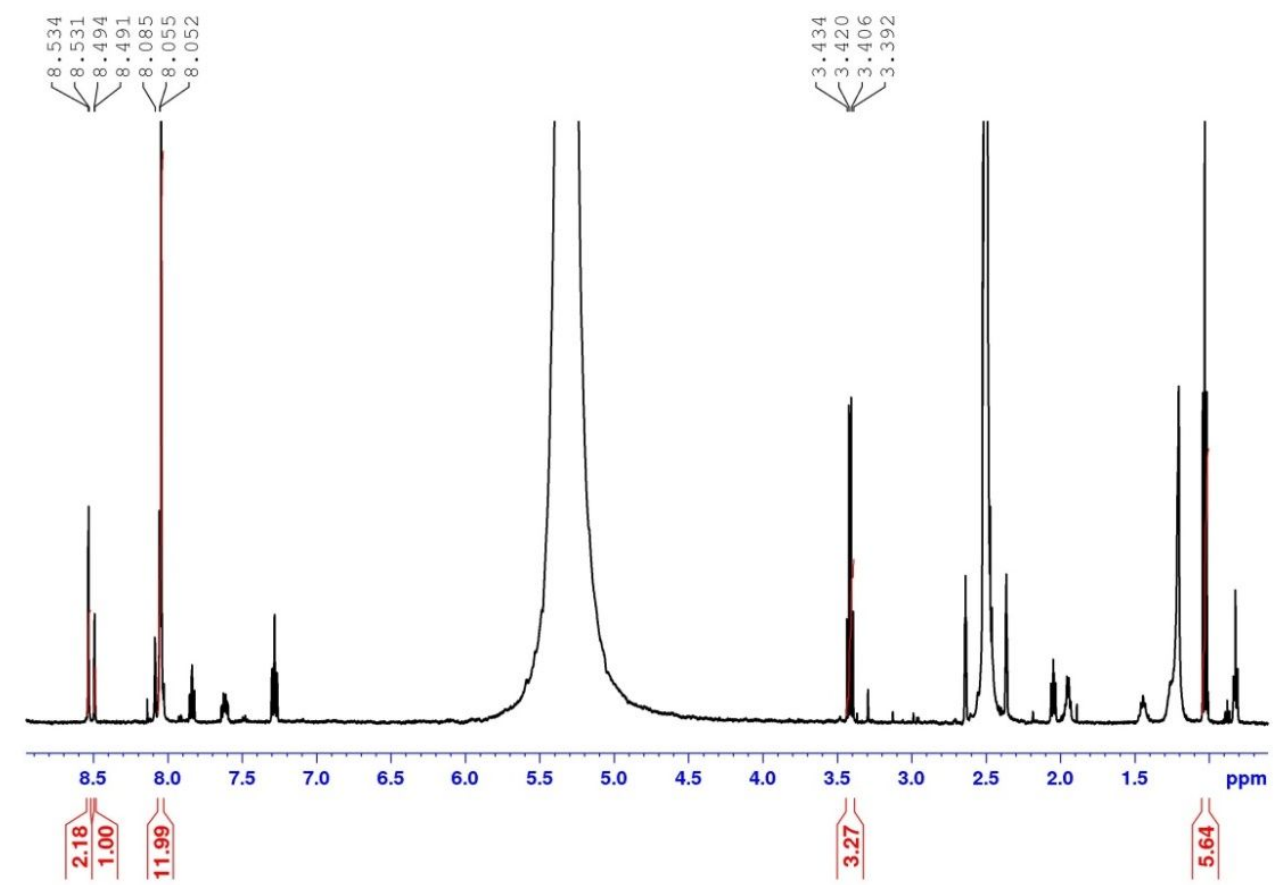

Figure S37. ${ }^{1} \mathrm{H}$ NMR spectrum of EtOH-exchanged Y-thc-MOF-1 (digested in $\mathrm{HCl} / \mathrm{DMSO}-\mathrm{d}_{6}$ solution), after overnight degas at $40^{\circ} \mathrm{C}$ under dynamic vacuum. The ratio of EtOH per cluster is 3.3:1. 


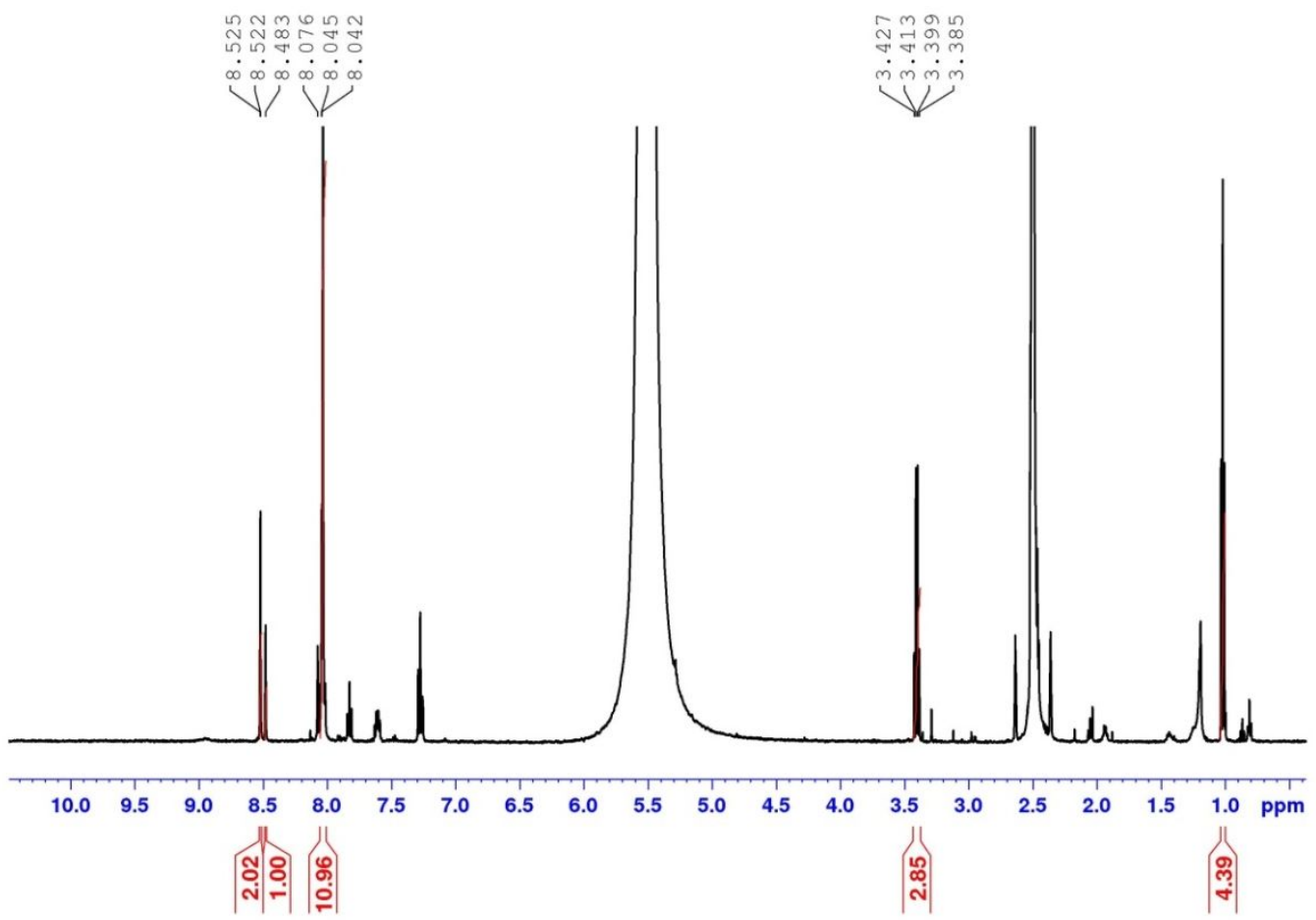

Figure S38. ${ }^{1} \mathrm{H}$ NMR spectrum of EtOH-exchanged Y-thc-MOF-1 (digested in $\mathrm{HCl} / \mathrm{DMSO}-\mathrm{d}_{6}$ solution), after overnight degas at $60^{\circ} \mathrm{C}$ under dynamic vacuum. The ratio of EtOH per cluster is 2.9:1.

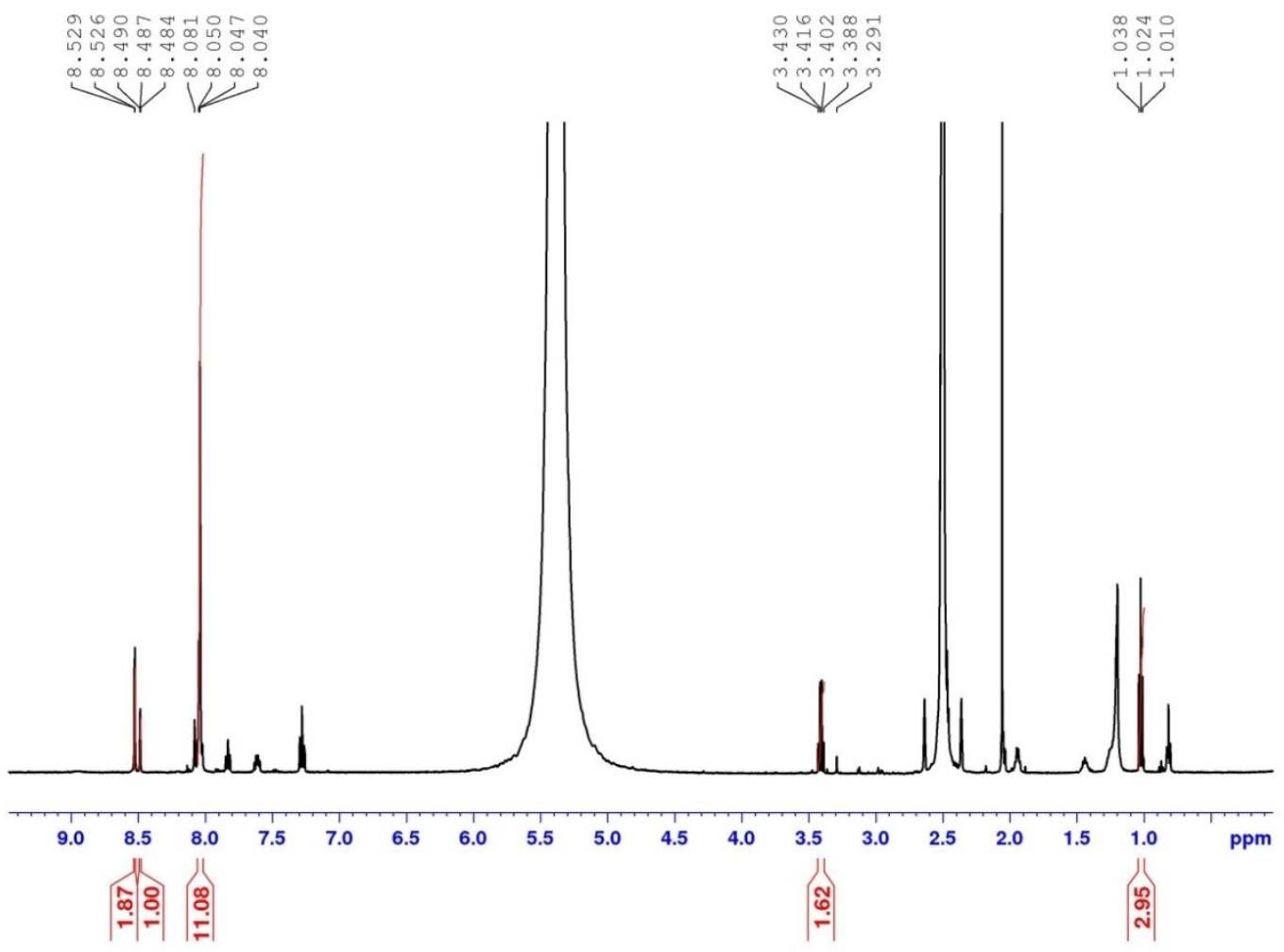

Figure S39. ${ }^{1} \mathrm{H}$ NMR spectrum of EtOH-exchanged Y-thc-MOF-1 (digested in $\mathrm{HCl} / \mathrm{DMSO}-\mathrm{d}_{6}$ solution), after overnight degas at $80^{\circ} \mathrm{C}$ under dynamic vacuum. The ratio of EtOH per cluster is 1.6:1. 


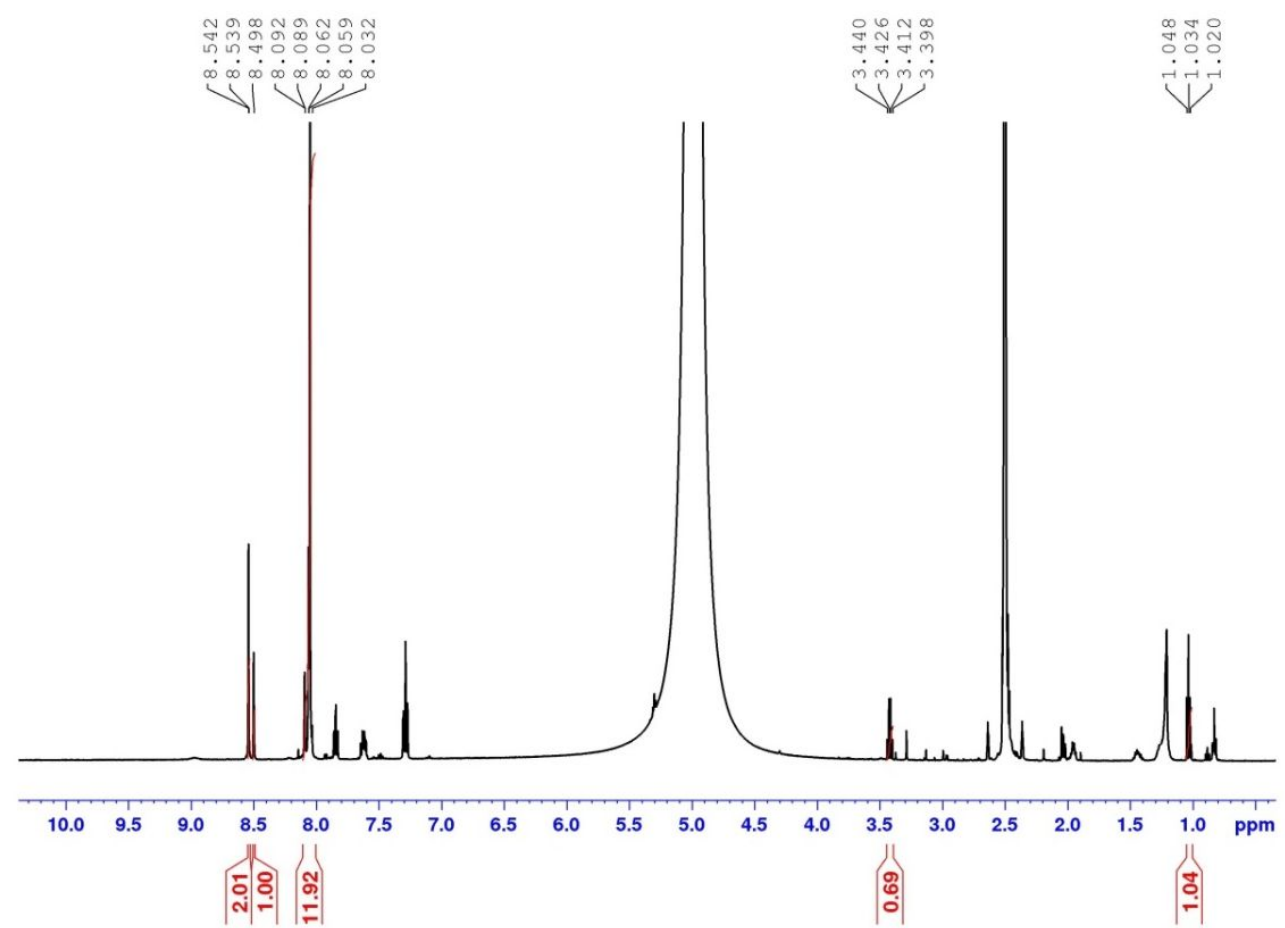

Figure S40. ${ }^{1} \mathrm{H}$ NMR spectrum of EtOH-exchanged Y-thc-MOF-1 (digested in $\mathrm{HCl} / \mathrm{DMSO}-\mathrm{d}_{6}$ solution), after overnight degas at $100^{\circ} \mathrm{C}$ under dynamic vacuum. The ratio of EtOH per cluster is 0.7:1.

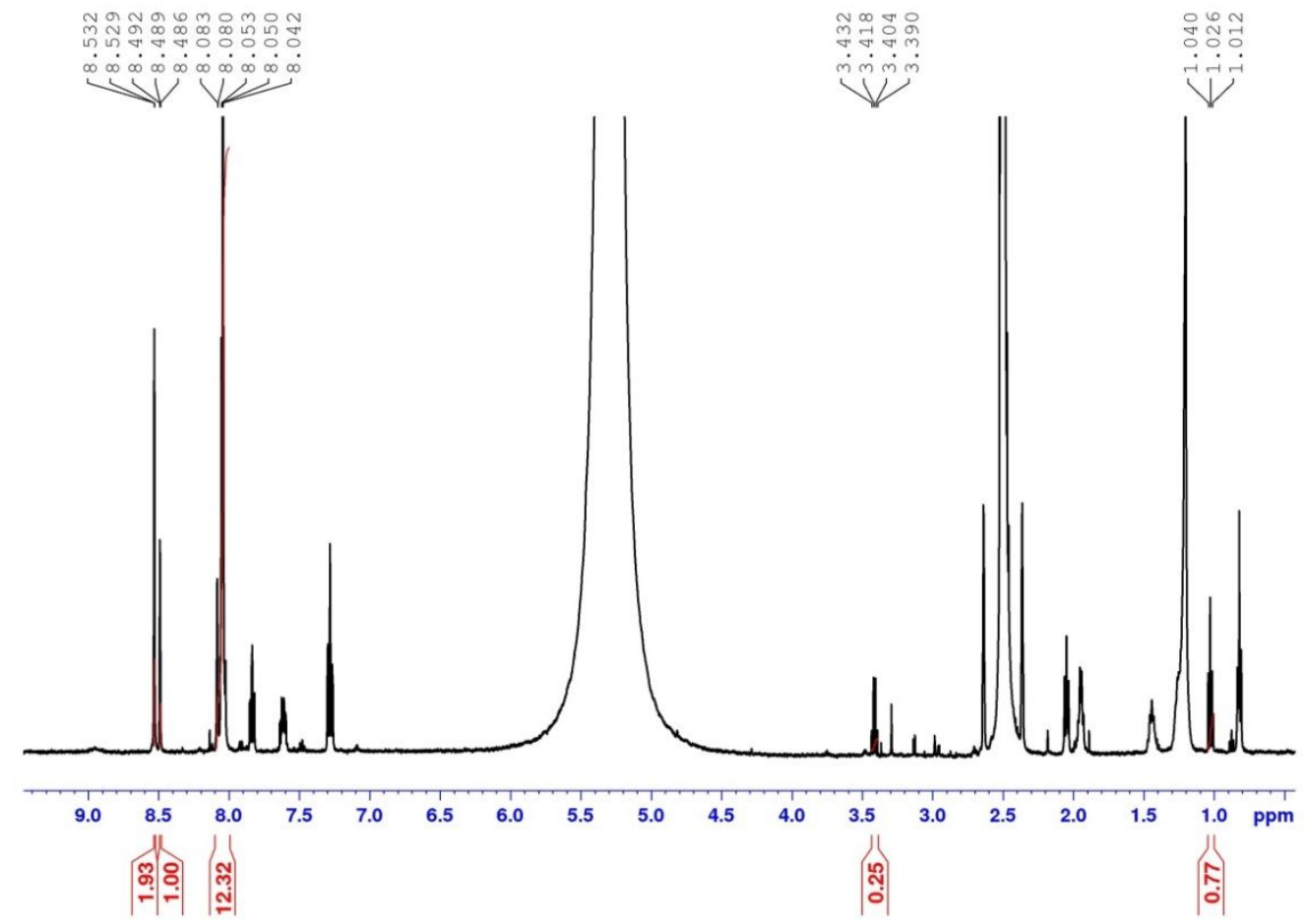

Figure S41. ${ }^{1} \mathrm{H}$ NMR spectrum of EtOH-exchanged Y-thc-MOF-1 (digested in $\mathrm{HCl} / \mathrm{DMSO}^{-\mathrm{d}_{6}}$ solution), after overnight degas at $120^{\circ} \mathrm{C}$ under dynamic vacuum. The ratio of EtOH per cluster is 0.25:1. 
EtOH exchanged Y-thc-MOF-1 before degas

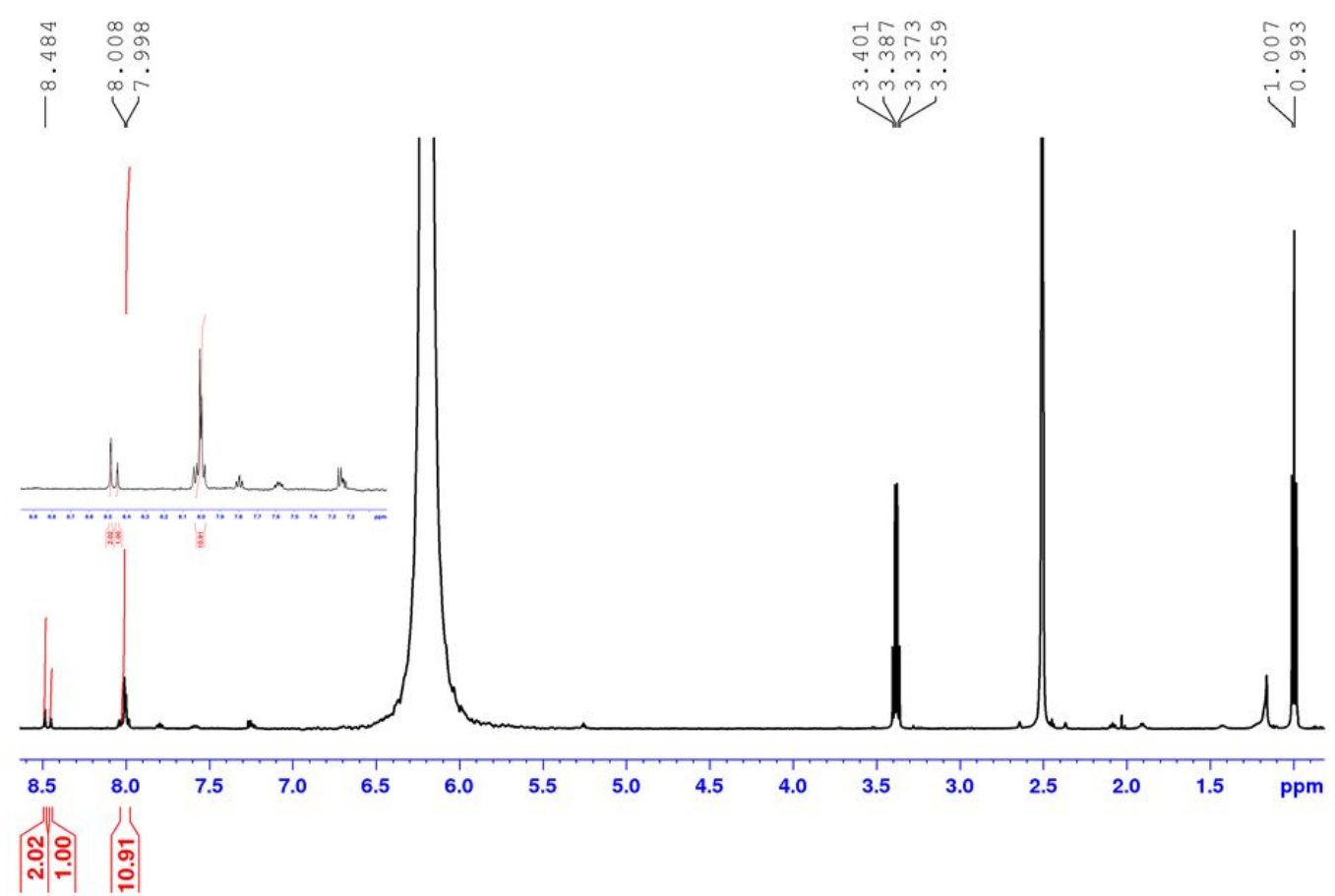

Figure S42. ${ }^{1} \mathrm{H}$ NMR spectrum of ethanol-exchanged Y-thc-MOF-1 (digested in $\mathrm{HCl} / \mathrm{DMSO}-\mathrm{d}_{6}$ solution), before degas. The spectrum confirmed the absence of DMF.

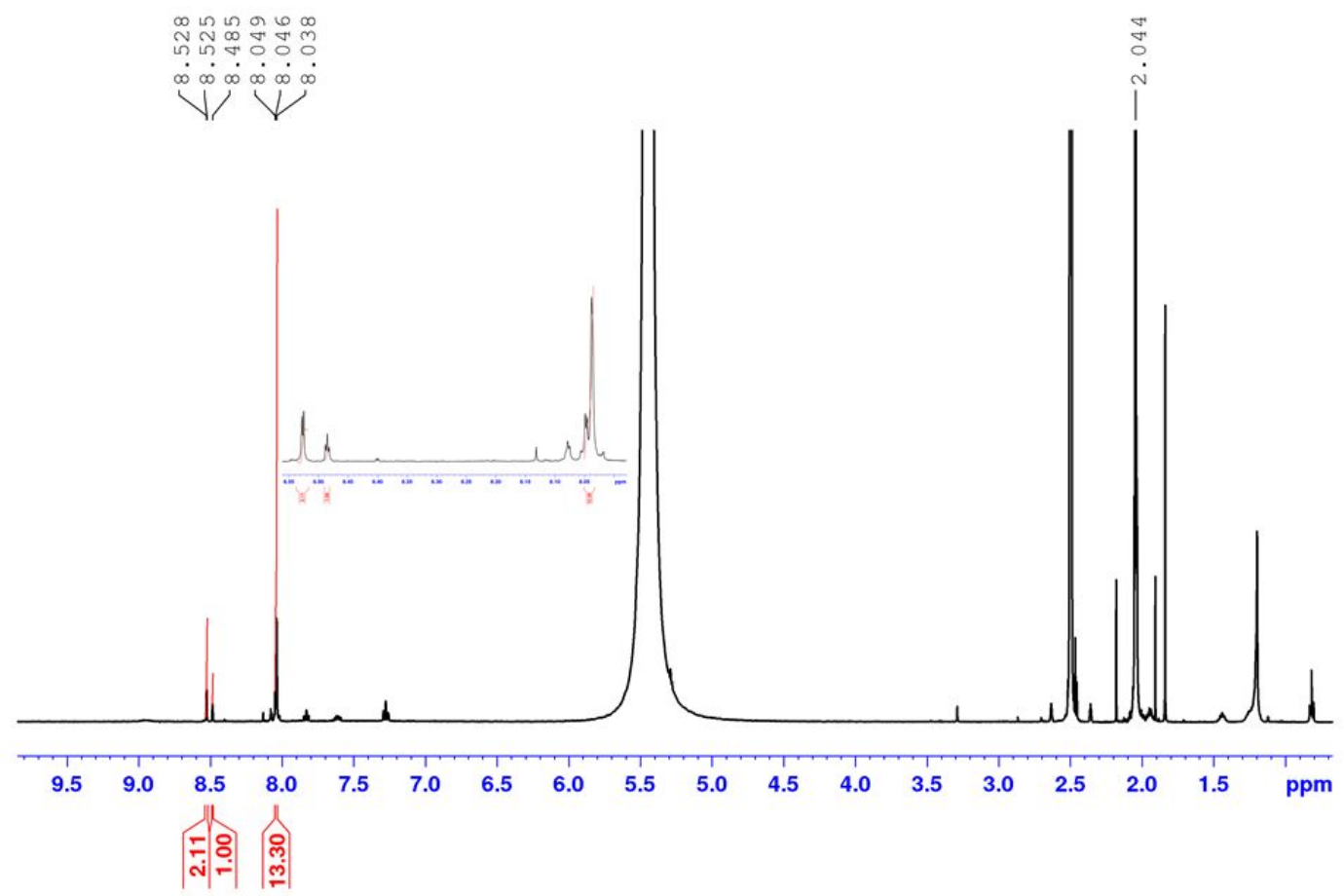

Figure S43. ${ }^{1} \mathrm{H}$ NMR spectrum of $\mathrm{CH}_{3} \mathrm{CN}$-exchanged Y-thc-MOF-1 (digested in $\mathrm{HCl} / \mathrm{DMSO}-\mathrm{d}_{6}$ solution). The spectrum confirmed the absence of DMF. 


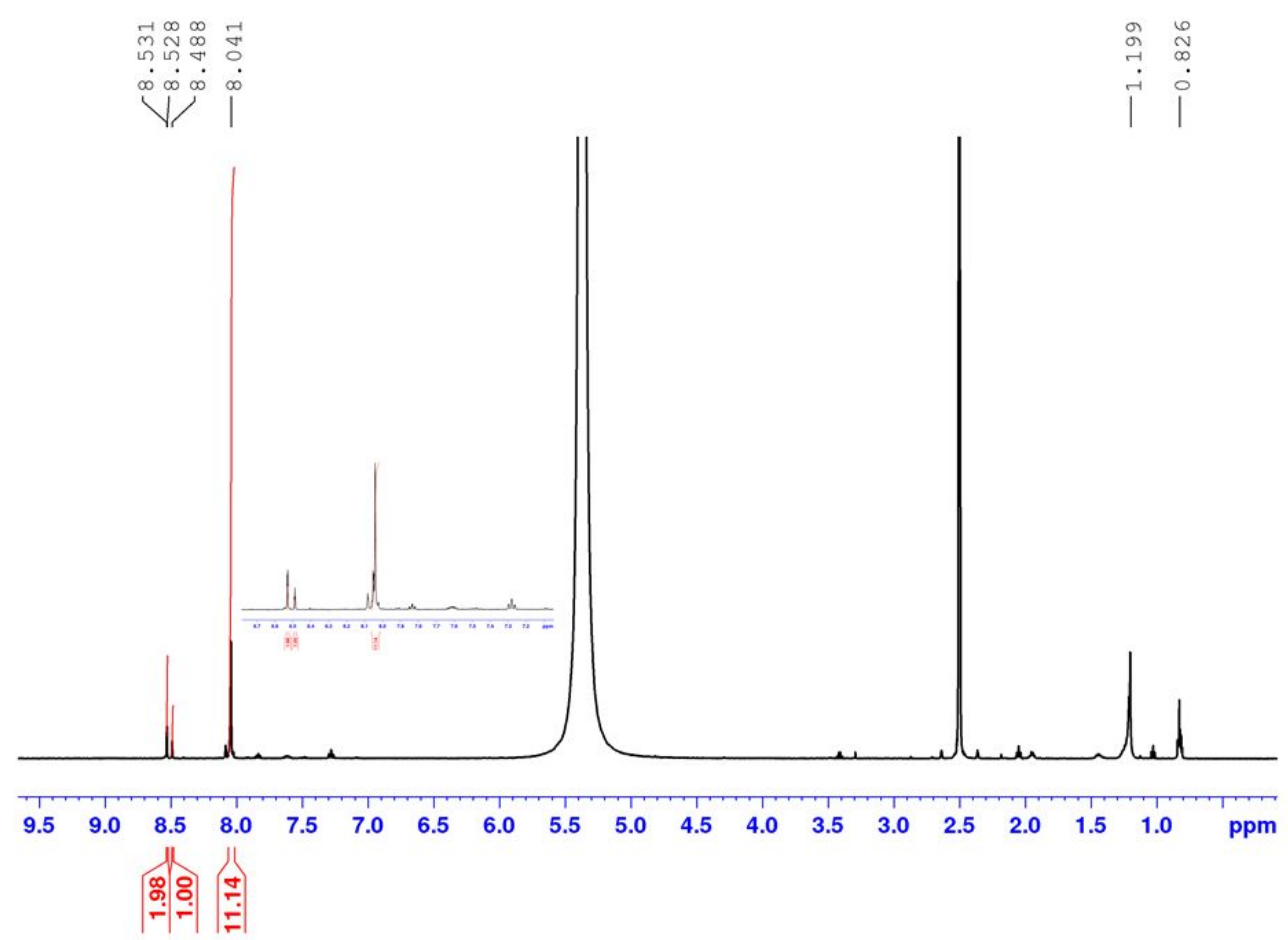

Figure S44. ${ }^{1} \mathrm{H}$ NMR spectrum of hexane-exchanged Y-thc-MOF-1 (digested in HCl/DMSO- $\mathrm{d}_{6}$ solution). The spectrum confirmed the absence of DMF.

\section{Thermal gravimetric analyses (TGA)}

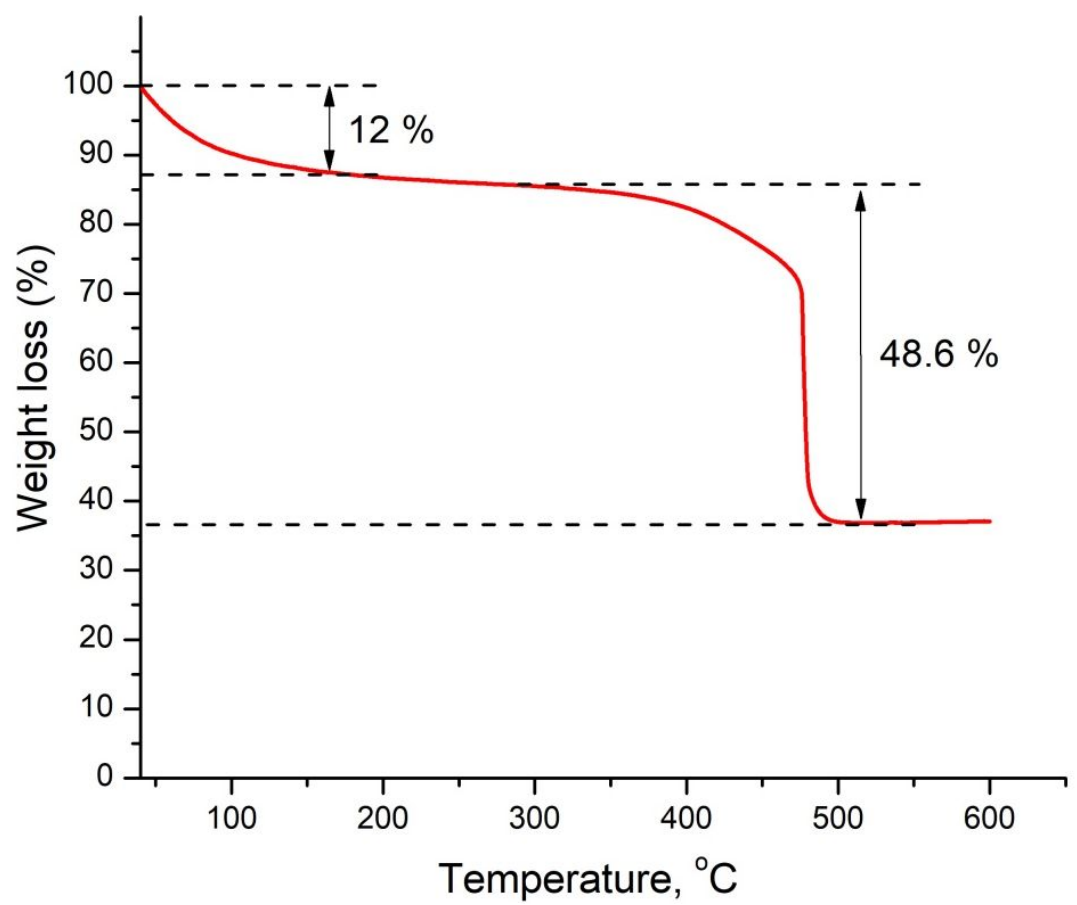

Figure S45. TGA curve of activated Y-thc-MOF-1 after ethanol exchange, recorded under nitrogen flow with a heating rate $5^{\circ} \mathrm{C} / \mathrm{min}$. The compound undergoes an initial weight loss of $12 \%$ up to 150 ${ }^{\circ} \mathrm{C}$ which is attributed to the removal of the coordinated ethanol molecules (four per $\mathrm{Y}_{6}$-cluster) and possibly some absorbed moisture due to exposure to open air before measurement. Thereafter, no 
noticeable weight loss occurs up to $\sim 280^{\circ} \mathrm{C}$ indicating that Y-thc-MOF-1 is thermally stable up to this temperature, which is consistent with the VT-PXRD results. Above $280{ }^{\circ} \mathrm{C}$ a progressive weight loss occurs with the main event observed in the range between $400-500{ }^{\circ} \mathrm{C}$ and attributed to the decomposition of the organic part of the framework. The observed total weight loss of $60.6 \%$ is lower compared to the calculated value of $71.3 \%$ based on the formula $\mathrm{Y}_{6}\left(\mu_{3}-\mathrm{OH}\right)_{8}(2-$ $\mathrm{FBA})_{3}(\mathrm{HCOO}) \mathrm{L}_{2}(\mathrm{EtOH})_{4}$, considering the formation of $\mathrm{Y}_{2} \mathrm{O}_{3}$ above $500{ }^{\circ} \mathrm{C}$ and suggests the presence of remaining carbon due to incomplete oxidation under nitrogen atmosphere.

\section{References}

${ }^{1}$ David Dubbeldam, Sofía Calero, Donald E. Ellis \& Randall Q. Snurr. RASPA: molecular simulation software for adsorption and diffusion in flexible nanoporous materials, Molecular Simulation, 2016 42:2, 81-101.

${ }^{2}$ A. K. Rappe, C. J. Casewit, K. S. Colwell, W. A. Goddard, and W. M. Skiff Journal of the American Chemical Society 1992114 (25), 10024-10035.

${ }^{3}$ Breneman, C.M. and Wiberg, K.B. (1990), Determining atom-centered monopoles from molecular electrostatic potentials. The need for high sampling density in formamide conformational analysis. J. Comput. Chem. 1990, 11: 361-373.

${ }^{4}$ Neese, F., Software update: the ORCA program system, version 4.0. WIREs Comput Mol Sci, 2018, 8: e1327.

${ }^{5}$ Dolomanov, O. V; Blake, A. J.; Champness, N. R.; Schröder, M. OLEX: New Software for Visualization and Analysis of Extended Crystal Structures. J. Appl. Crystallogr. 2003, 36, 1283-1284.

${ }^{6}$ Sheldrick, G. M. SHELXT - Integrated Space-Group and Crystal-Structure Determination. Acta Crystallogr. Sect. A 2015, 71 (1), 3-8

${ }^{7}$ Sheldrick, G. M. A Short History of SHELX. Acta Crystallogr. Sect. A 2008, 64, 112-122.

${ }^{8}$ Sheldrick, G. M. Crystal Structure Refinement with SHELXL. Acta Crystallogr. Sect. C 2015, 71 (1), 38. 